\title{
WHY SING AND DANCE? \\ AN EXAMINATION OF THE COOPERATIVE EFFECTS OF GROUP SYNCHRONY
}

BY

PAUL REDDISH

\begin{abstract}
A thesis
submitted to the Victoria University of Wellington in fulfilment of the requirements for the degree of Doctor of Philosophy
\end{abstract}

Victoria University of Wellington

2012 


\begin{abstract}
The universality and antiquity of music and dance suggest that they may serve some important adaptive function. Why are music and dance cultural universals? One popular theory is that music and dance function to enhance mutually benefiting cooperation. While the cooperation hypothesis finds support from anthropological observations and recent experiments, the proximate mechanisms remain unclear. In this thesis, I examine if being in synchrony is a critical factor underlying music and dance's cooperative effects. I define synchrony as rhythmically moving or vocalising in time with others. In support of synchrony's role in fostering cooperation, a number of studies exploring two person interactions have found positive social effects from synchrony. However, it is not clear whether synchrony enhances cooperation in groups larger than two as typical with music and dance.
\end{abstract}

This thesis describes five laboratory experiments that were conducted to investigate: (1) whether group synchrony increases cooperation; and (2) which psychological mechanisms are involved in producing synchrony's cooperative effects. In the first three experiments, small groups of participants were asked to perform body movements or to vocalise words in time with the same (synchrony condition) or different (asynchrony condition) metronome beats. Cooperative behaviour was measured with a helping scenario and an economic game. A small increase in cooperation was found with synchronous movement compared to asynchronous movement (experiment 1 and 3). However, this difference was only significant with the economic game measure (experiment 3). When vocalisation was isolated (experiment 2), contrary to expectations, the highest level of helping occurred after the asynchrony vocal condition.

A plausible explanation for such small and inconsistent effects comes from the method in which synchrony was manipulated. Following previous methodologies, the goal for participants was to entrain to their own beat. Yet in natural human ecologies, synchrony is a product of shared intentionality - the sharing of psychological states to produce collaborative behaviour. To better understand the contribution of shared intentionality, experiments 4 and 5 varied synchrony with shared intentionality, and then measured cooperation. These experiments revealed that when participants worked together to create synchrony, substantial increases in cooperation were found, for both synchronous vocalisations (experiment 4) and for synchronous movements (experiment 5). Synchrony was 
also found to significantly amplify two key hypothesised mediating variables: perceived similarity and entitativity (the degree to which a collection of people are perceived as a group). Path analysis supported a proposed mechanism by which synchrony combines with shared intentionality to produce greater cooperation through: (1) increased attention to the behaviours of other participants; and (2) reinforcement of successful cooperation.

This thesis, therefore, extends previous research on group music and dance in three ways. First, the combined effect of synchrony and shared intentionality is identified as critical to the cooperation enhancing effects of music and dance. Second, it describes plausible mechanisms for how synchrony may lead to increased cooperation. Third, it provides empirical evidence in support of these mechanisms. 


\section{Acknowledgements}

My two supervisors Dr Ronald Fischer and Dr Joseph Bulbulia contributed enormously to this thesis. It was due to Joseph's encouragement that I gained the confidence to do this $\mathrm{PhD}$. Joseph also provided the initial inspiration for the topic of this thesis, along with many other brilliant ideas, insights and comments. Ron provided sage-like wisdom and knowledge whenever needed, even when he was on the other side of the world. Both also provided funding through research grants they had obtained. Thank you Ron and Joseph for your help and enthusiasm, I really enjoyed working with you and I hope we can collaborate on future projects together.

The experiments I conducted would not have been possible without the help of a number of people who were happy to volunteer their time. I'd like to thank in alphabetical order: Aleece Brady, Rohan Callander, Pollyane Diniz, Amit Dutta, Alexis Garland, Adrienne Girling, Ursula Haflinger, Julie Heidrich, Nora Hellberg, Christina Janus, Carolin Konrad, Bee Lim, Elena Maydell, Tim McVicar, Rachel Palmer, Shachi Phadke, Hanne Schenk, Solveig Schudeiske, Floor Spijkers. Also thank you to all the participants who gave their time to be a part of this research.

The staff in the psychology department provided valuable assistance. Doug Drysdale helped acquire or build the equipment used in the experiments. Vijayarengan Ramanuja Chary programmed the software used in experiment 5 to record the foot-pedal data. Ngaire Lavery, Jebi Jayapalan, and Wendy Ward provided important administrative support. Thanks to Victoria University of Wellington who funded my $\mathrm{PhD}$ with a Victoria Doctoral scholarship and a Victoria PhD Submission scholarship.

I would like to thank my parents Steve Reddish and Cathy Reddish, who as well as providing constant encouragement and emotional support during the $\mathrm{PhD}$ process, provided me the intellectual, financial, and emotional resources whilst growing up that enabled me to get to where I am today. Special thanks also to Terrie Reddish for the support she gave me. Finally, I would like to thank Penny Tok who has been by my side during this very challenging endeavour. Penny provided much valuable advice, hands on help, and most importantly her abundant love and kindness. 


\section{Table of contents}

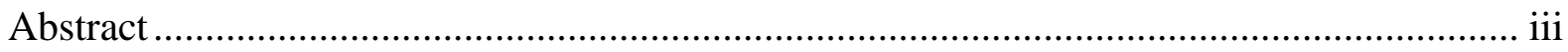

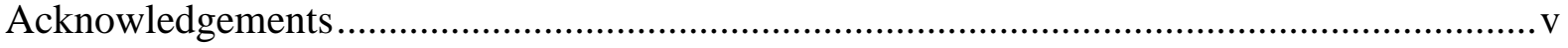

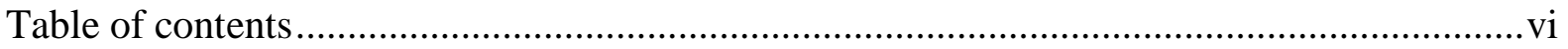

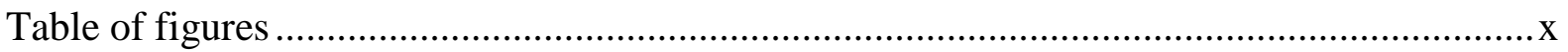

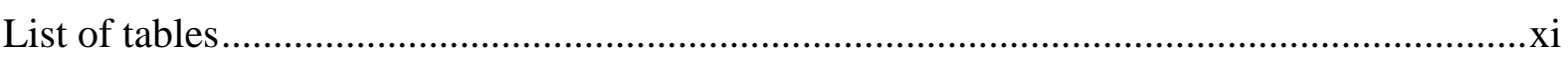

Chapter 1

THE AIMS AND THE APPROACH OF THE THESIS …............................................ 1

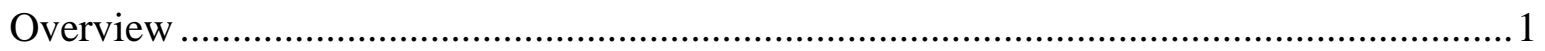

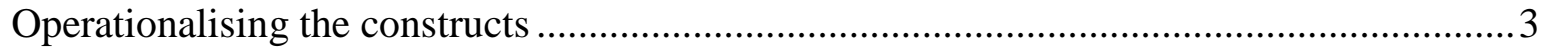

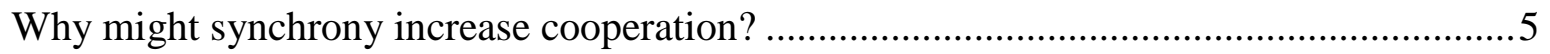

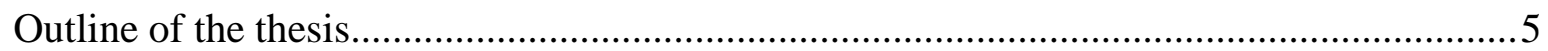

Chapter 2

INTERACTIONAL SYNCHRONY IN HUMANS .....................................................

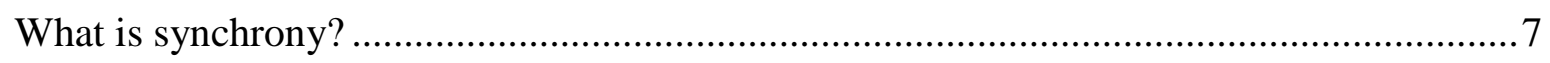

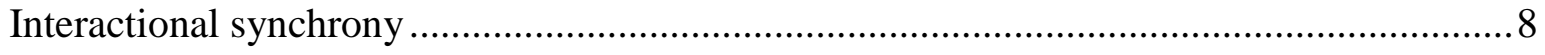

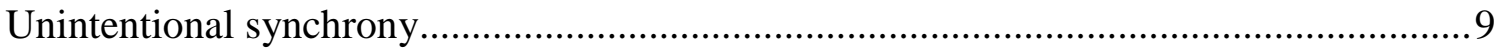

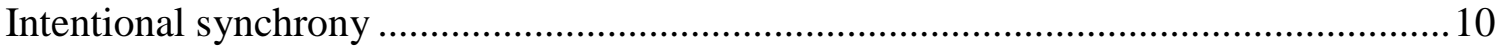

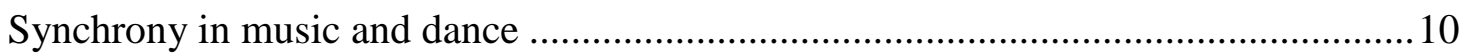

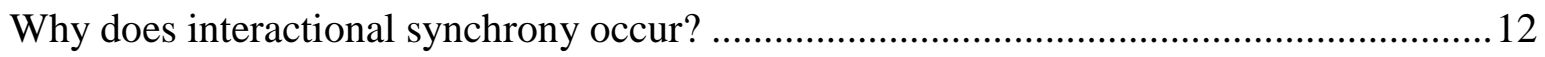

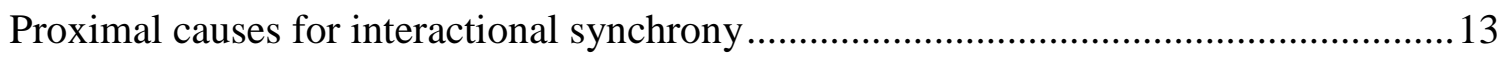

Coordination aid for collaborative activities............................................................ 13

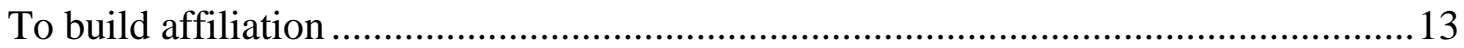

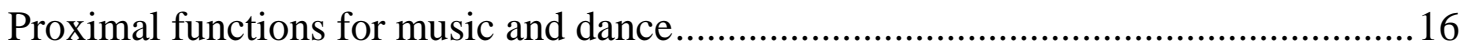

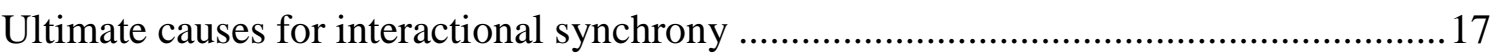

Chapter 3

THE MUSIC/DANCE-COOPERATION HYPOTHESIS ...................................................21

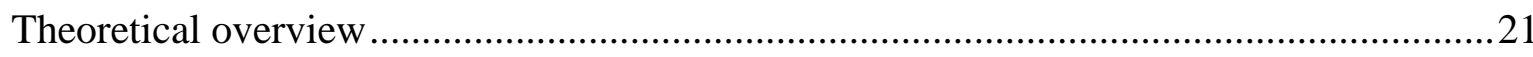

Anthropological and historical evidence for the music/dance-cooperation hypothesis .......23

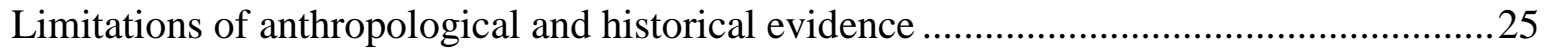

Experimental evidence for the music/dance-cooperation hypothesis ...............................26

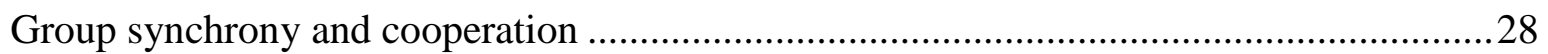


Where to go from here?

Chapter 4

SYNCHRONY UNDER MINIMAL CONDITIONS

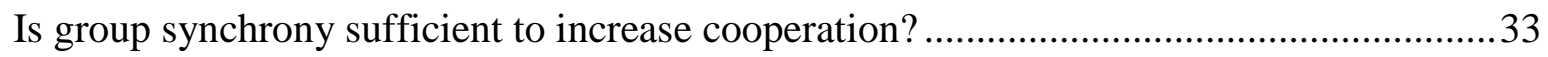

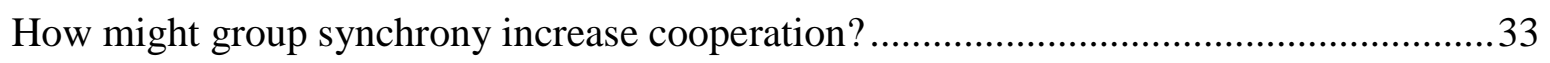

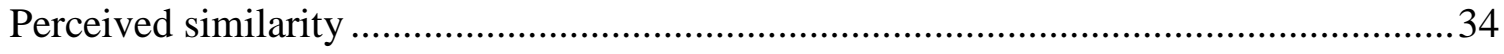

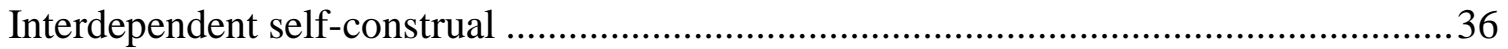

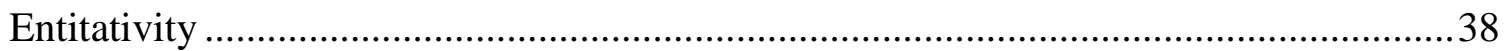

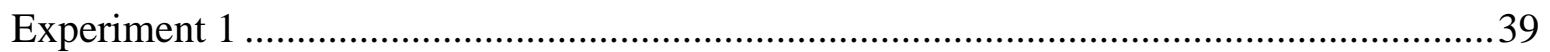

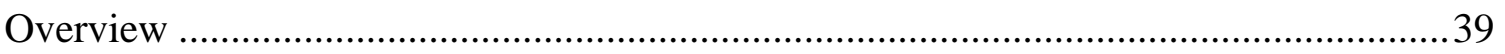

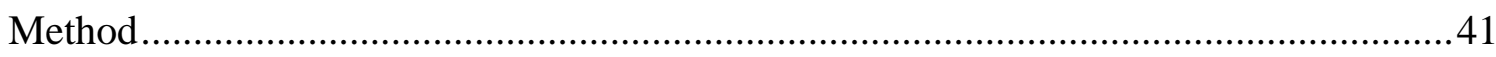

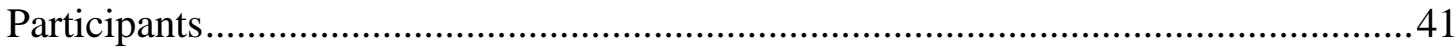

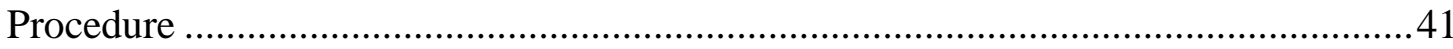

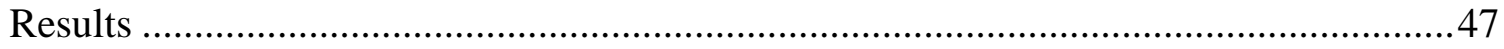

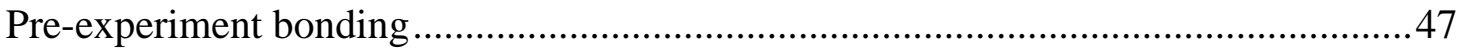

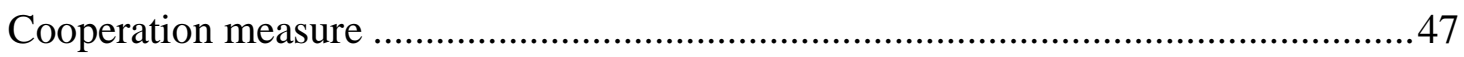

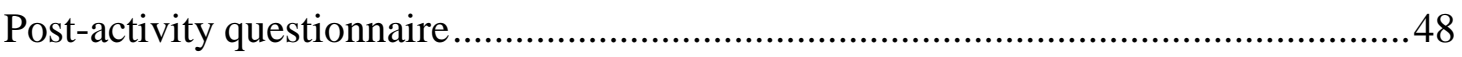

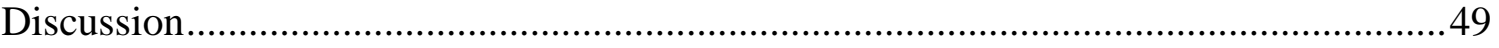

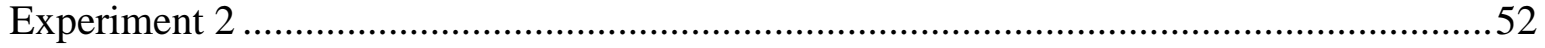

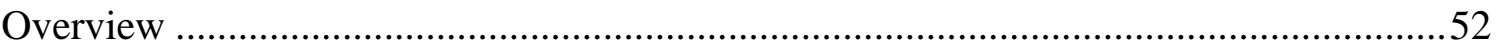

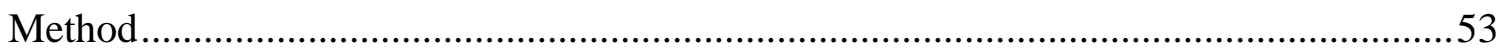

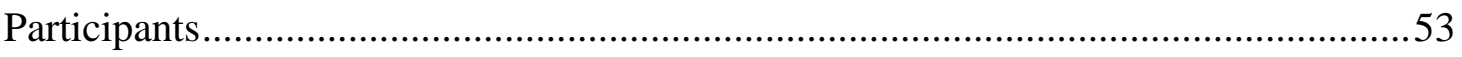

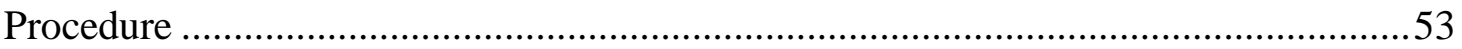

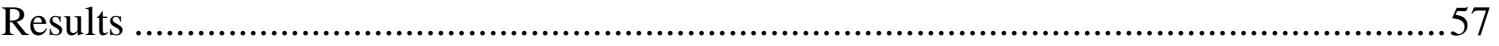

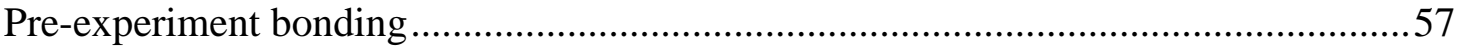

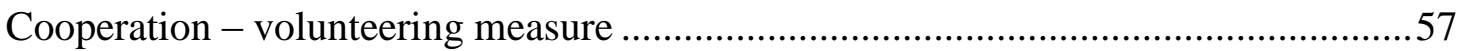

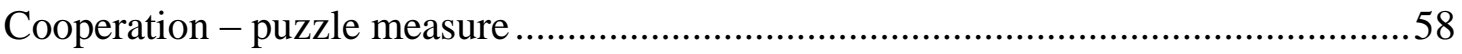

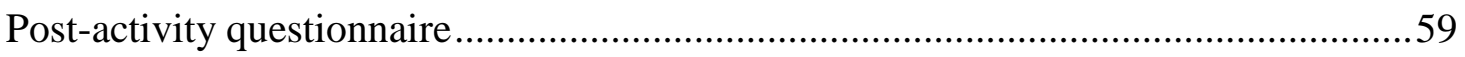

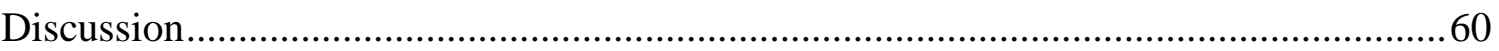

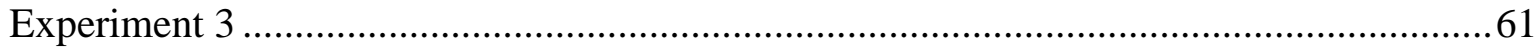

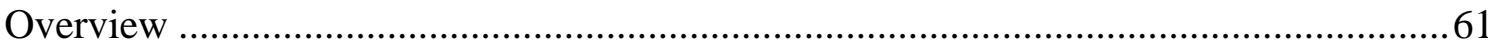

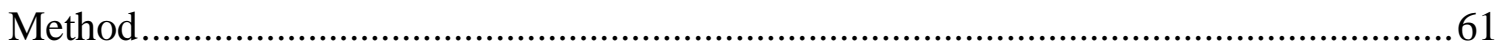

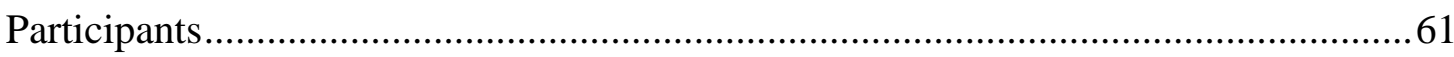


Procedure

Results

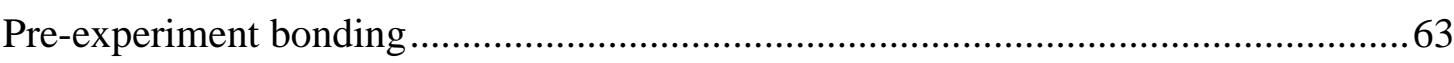

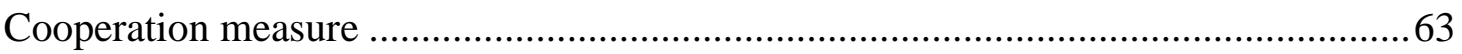

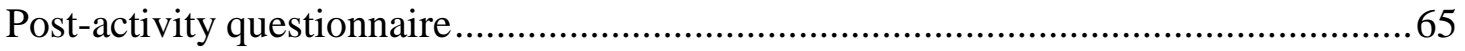

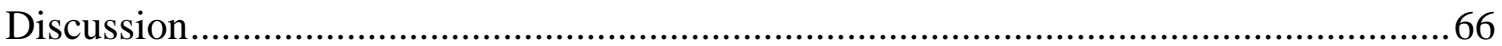

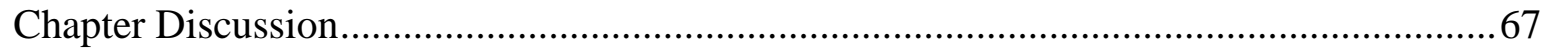

Chapter 5

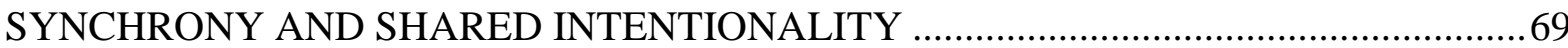

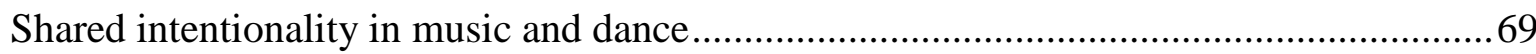

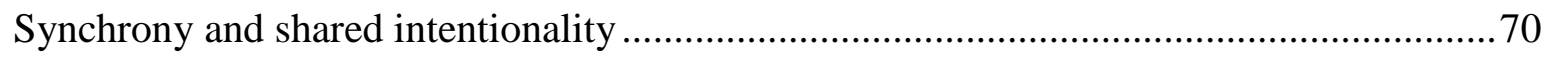

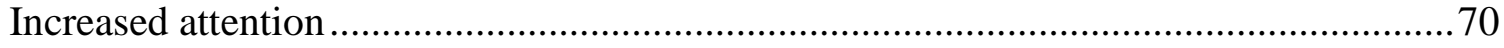

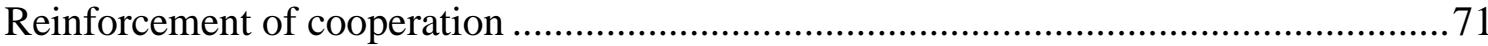

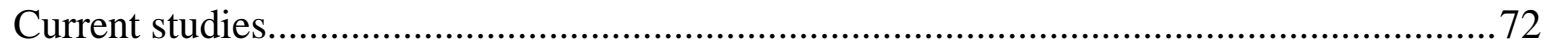

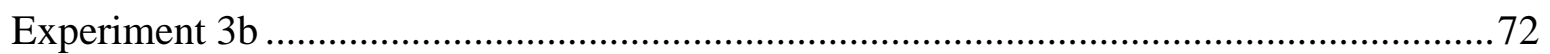

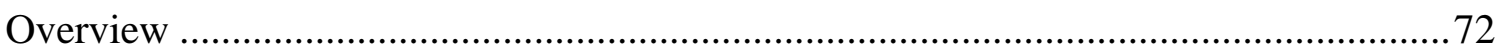

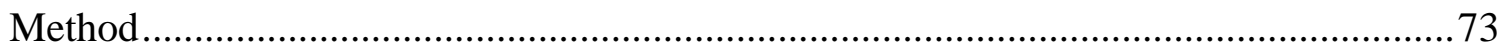

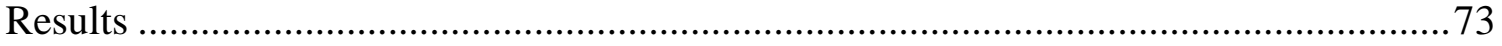

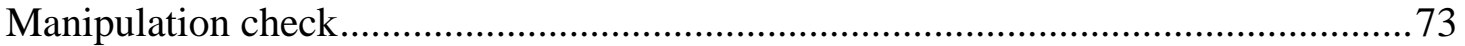

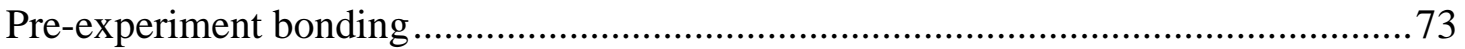

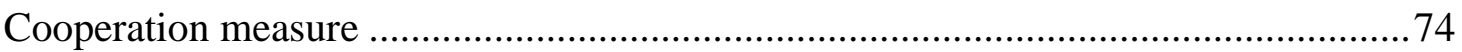

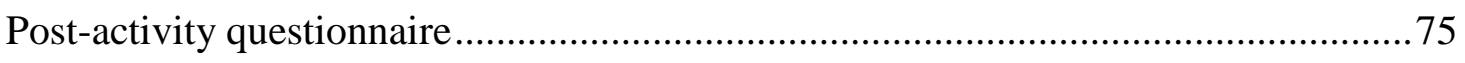

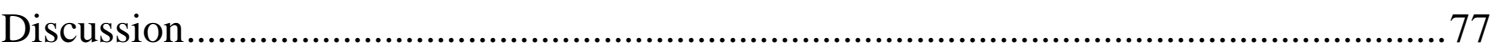

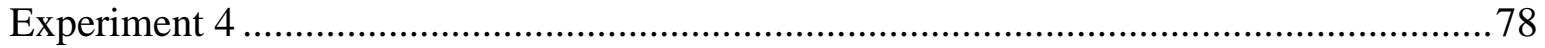

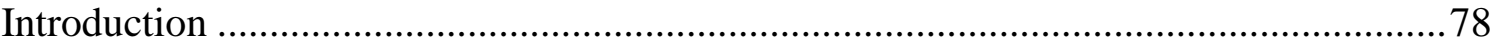

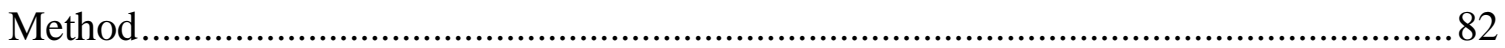

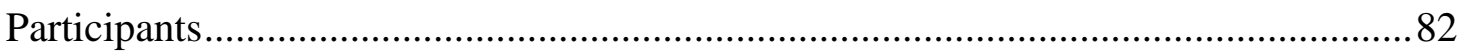

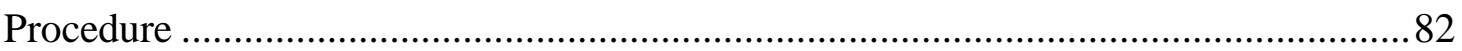

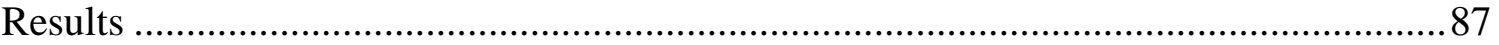

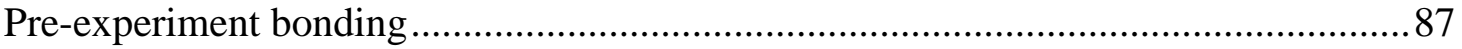

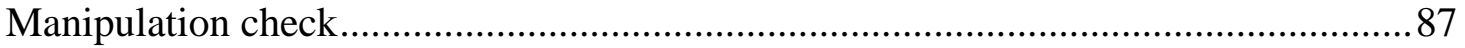

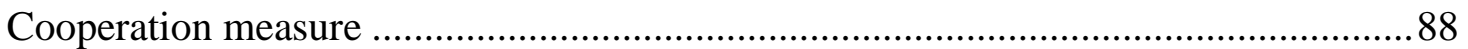

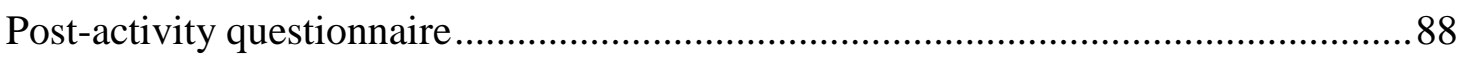




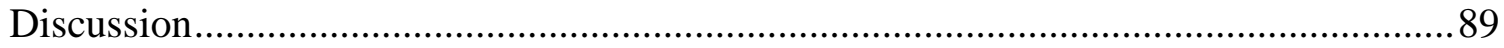

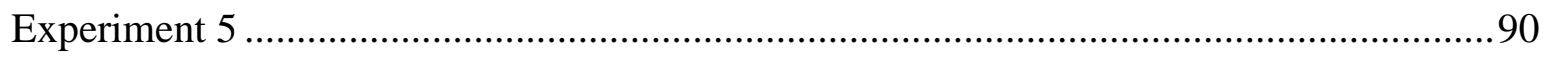

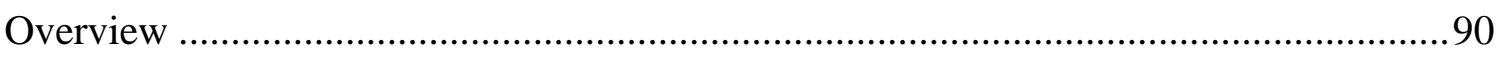

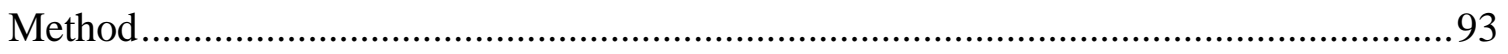

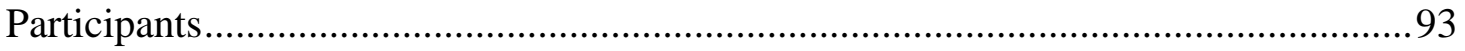

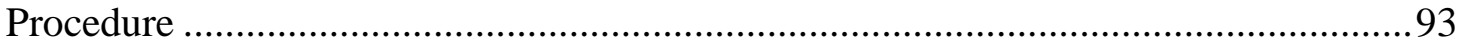

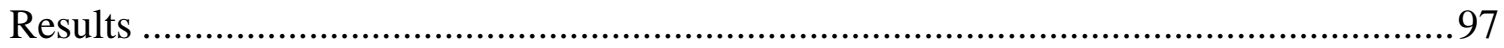

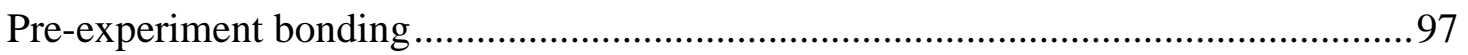

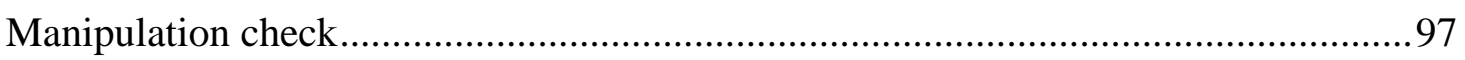

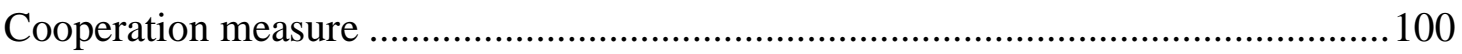

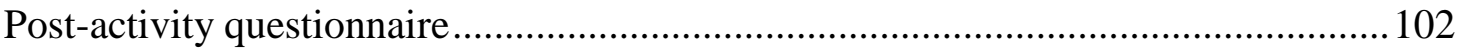

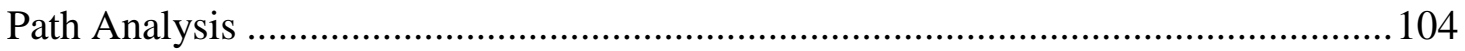

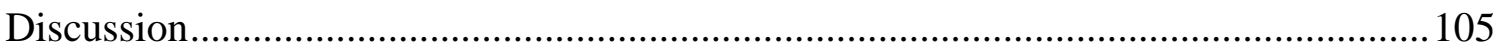

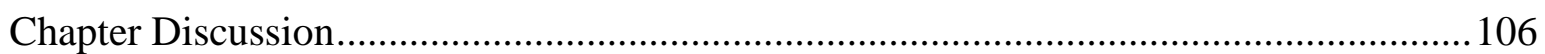

\section{Chapter 6}

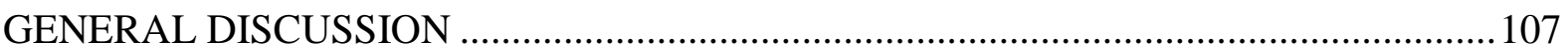

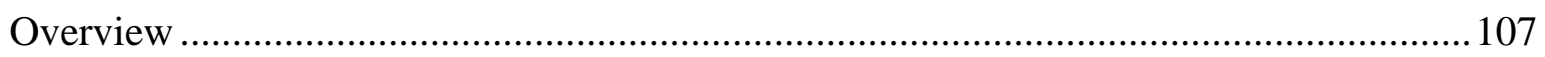

Does group synchrony lead to greater cooperation? ...................................................... 107

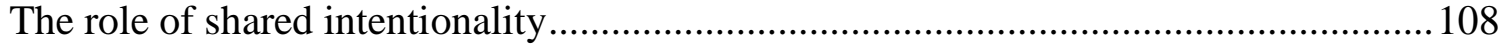

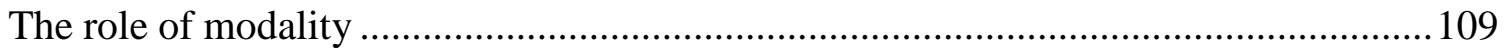

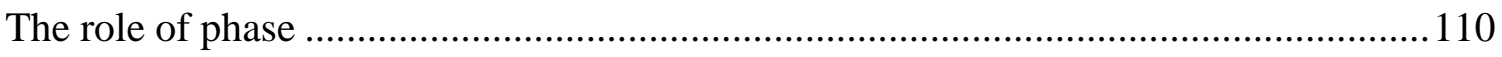

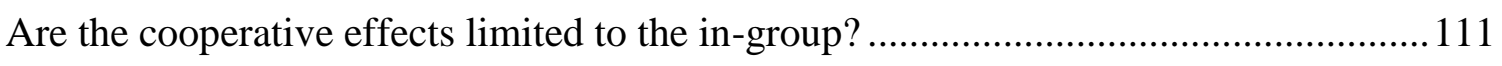

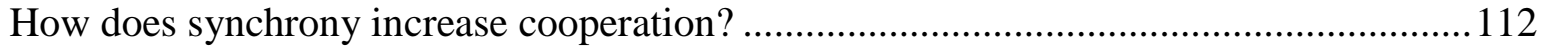

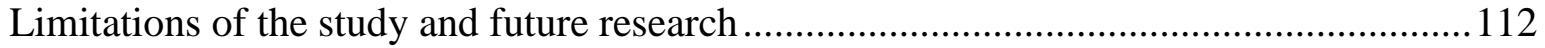

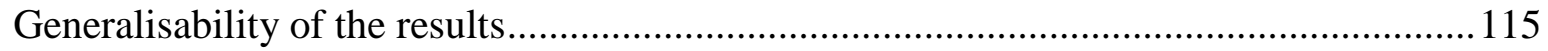

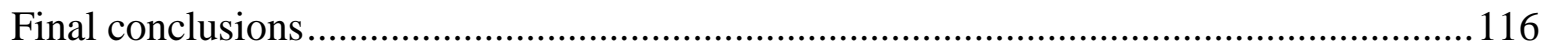

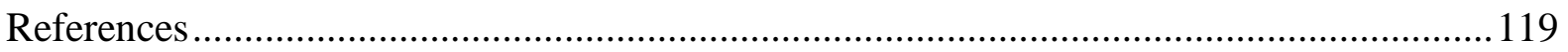

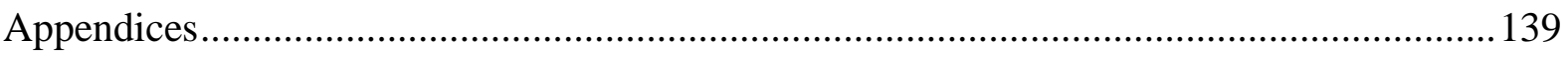

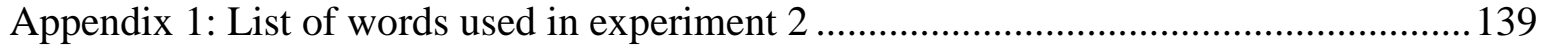

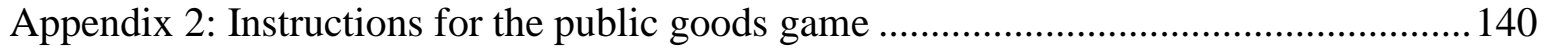

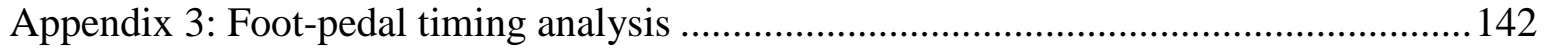

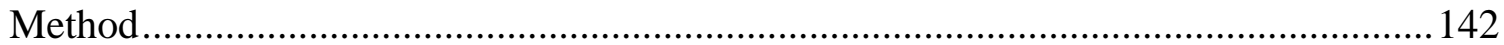

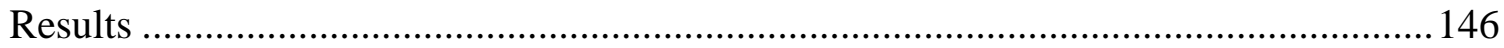




\section{Table of figures}

Figure 1. The Inclusion of Other in the Self scale (IOS). .46

Figure 2. Mean amount of time willing to donate (minutes) for each of the movement conditions. 48

Figure 3. Photograph of the jigsaw puzzle used in the puzzle cooperation measure. .56

Figure 4. Mean amount of time willing to donate (minutes) for each of the vocalising conditions .58

Figure 5. Percentage of participants who spontaneously helped during the puzzle task for each vocalising condition.

Figure 6. Mean amount of money place into the group investment (dollars) for each of the movement conditions

Figure 7. Percentage of participants that placed all of their money into the group account for each movement condition.

Figure 8. Mean level of entitativity for each of the movement conditions

Figure 9. Mean amount of money placed into the group investment (dollars) for the new

shared goal condition compared to the original three conditions from experiment 3 ..

Figure 10. Histogram of the distribution of the amount of money (dollars) placed into the group investment for the shared goal condition

Figure 11. Percentage of participants that placed all of their money into the group account for the new shared goal condition compared to the original three conditions from experiment 3.

Figure 12. Mean interdependent self-construal for the new shared goal condition compared to

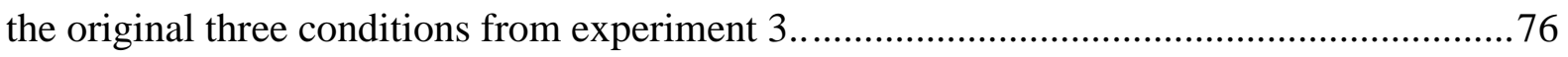

Figure 13. Modified Inclusion of Other in the Self scale (IOS) ......................................... 86

Figure 14. Percentage of participants choosing the cooperative Y option for each of the two

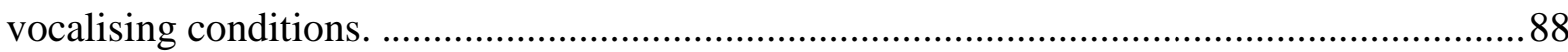

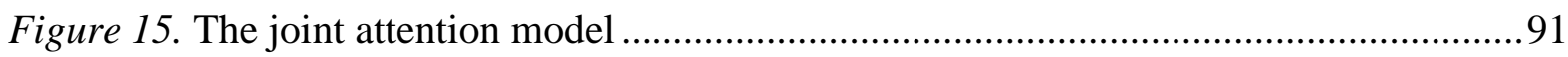

Figure 16. The reinforcement of cooperation model........................................................... 91

Figure 17. The combined joint attention and reinforcement of cooperation model ...............91

Figure 18. Photograph of the foot-pedal used in experiment 5 ..........................................96

Figure 19. Mean perceived synchrony for when synchronous, sequential and asynchronous movement is created through a group goal or an individual goal. 
Figure 20. Mean perceived cooperation for when synchronous, sequential and asynchronous movement is created through a group goal or an individual goal.

Figure 21. Percentage of participants choosing the cooperative Y option for when synchronous, sequential and asynchronous movement is created through a group goal or an

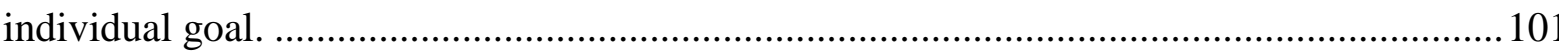

Figure 22. Mean entitativity for each of the movement conditions. .................................... 102

Figure 23. Mean perceived similarity for each of the movement conditions....................... 103

Figure 24. Mean attention directed towards the other participants for when synchronous, sequential and asynchronous movement is created through a group goal or an individual goal.

Figure 25. Standardised coefficients for the combined joint attention and reinforcement of cooperation model. 105

Figure 26. Vector diagram showing the distribution of $\bar{\theta}$ for each participant in the synchrony condition.

Figure 27. Vector diagram showing the distribution of $\bar{\theta}$ for each participant in the asynchrony condition.

Figure 28. Angular histogram showing the distribution of the relative phase angle $\theta$ for each pedal press for a participant in the asynchrony group goal condition (AsGG).

Figure 29. Vector diagram showing the distribution of $\bar{\theta}$ for each participant in the sequential condition. 150

\section{List of tables}

Table 1. Participants' choices for the stag hunt game. .84

Table 2. Summary of the six conditions and the goals participants were given..... .94 


\section{Chapter 1}

\section{THE AIMS AND THE APPROACH OF THE THESIS}

\section{Overview}

Human behavioural diversity is daunting. Our ability to imitate, to create novel behaviour, and to share mental states with others has led to an explosion of different cultural practices (Tomasello, Carpenter, Call, Behne, \& Moll, 2005). However, human behaviour is not infinite in its variance. Because of our shared history, our similar biological and cognitive makeup, and the fact that we face similar problems for survival and reproduction, human cultural behaviours often fall into similar clusters (Norenzayan \& Heine, 2005). One such cluster is the behaviour of humans to come together in groups to rhythmically move or vocalise in a highly coordinated fashion. Such behaviours are commonly classified under the terms music or dance.

The general aim of this thesis is to contribute towards explanations for why music and dance exist in the human species. Previous explorations of the function of music and dance have primarily been conducted in the domain of anthropology. Anthropological observation has generated a number of hypotheses about the function of music and dance (e.g., Durkheim, 1915/1965; Hanna, 1987; Radcliffe-Brown, 1948) but the field has lacked the empirical rigour to effectively test these theories (Bulbulia \& Reddish, 2012). For this thesis, I instead used experimental methods to test specific hypotheses that have been generated from real world observation of music and dance.

One challenge in investigating music and dance is that these terms describe a very wide range of behaviours (Merriam, 1964; Nettl, 1983). This is both due to issues with the categories music and dance which are very fuzzy and can be somewhat subjective (Bispham, 2006; I. Cross, 2001; Hanna, 1987) and to the fact that music and dance are so open to innovation and adoption for any number of human aesthetic tastes, needs, and functions (e.g., Merriam, 1964). In this thesis I focused on group production of music and dance in a ritualised context. I concentrated on group production of music and dance because music and dance are most commonly expressed as a social behaviour and appear particularly designed to aid in coordinating the behaviours of numerous people (see Kirschner \& Tomasello, 2009). By ritualised, I mean expressions of music and dance where the proclaimed goal for creating 
the song or dance is divorced from any functional utility for being in time (Boyer \& Lienard, 2006). Such ritualised group music and dance is very common in all cultures around the world (Blacking, 1973; D. Brown, 1991; Lomax, 1968). Musical artefacts also suggest music and dance - in some form - has been around for at least 40,000 years in the human species (Adler, 2009; Conard, Malina, \& Munzel, 2009). This ubiquity and history, on the one hand, but a lack of any obvious functional utility, on the other hand, render music and dance particularly puzzling and in need of scientific research.

Because of music's and dance's complexity and variation, it is unlikely that one theory can be used to describe all dimensions and instances of music and dance (S. Brown, 2000). As experimental techniques require the testing of specific hypotheses generated from a particular theory, here I concentrated on just one proposed function for music and dance: that music and dance fosters cooperation (the music/dance-cooperation hypothesis). This theory was chosen because it is currently one of the most popular theories (e.g., Freeman, 2000; McNeill, 1995; Roederer, 1984) and also because it provides an ultimate explanation for the existence of music and dance in the human species. A wide range of proximal functions have been described for why an individual might engage in music and dance that vary widely from culture to culture (e.g., Merriam, 1964). By concentrating on an ultimate explanation I can help narrow down those factors that may have been crucial in the initial development of music and dance, its ubiquity, and its conservation across millennia. The factors important in ultimate explanations, although not explaining all of the variance we currently see in expressions of music and dance, may explain some of the fundamental elements of music and dance.

Finding evidence for ultimate explanations is difficult as we cannot go back in time to assess what evolutionary pressures led to its selection or development (Buller, 2005). Ultimate explanations for human behaviours are also particularly problematic due to the interaction between cultural and biological evolution with models and theories looking to understand their interaction still in development (Boyd, Richerson, \& Henrich, 2011; Griffiths \& Gray, 2005; Richerson \& Boyd, 2005). The aim of this thesis is not to provide theoretical justification for ultimate explanations of music and dance as this has been discussed elsewhere (Bispham, 2010; I. Cross, 1999, 2001, 2003, 2009; Hauser \& McDermott, 2003; Huron, 2001; Mithen, 2005; Wallin, Merker, \& Brown, 2000). Instead, I aim to provide data consistent with the ultimate explanation that music and dance increases cooperation. Importantly, this theory provides an experimentally testable and falsifiable 
hypothesis: performance of group music and dance should lead to greater levels of cooperation.

\section{Operationalising the constructs}

Experimental methods require isolating a variable of interest, manipulating that variable while holding other factors constant, and measuring the effect on another variable of interest. To conduct an experiment, the construct being manipulated (the independent variable) as well as the construct being measured (the dependent variable) need to be operationalised. Operationalising music and dance is problematic due to the aforementioned variation in its manifestations. One method is to get participants to perform what is usually culturally defined as music or dance and compare the effects of this to a control condition of an activity which is not usually culturally defined as music or dance (as per Anshel \& Kipper, 1988; Kirschner \& Tomasello, 2010). As well as potential variance in cultural definitions of music and dance, such an approach can result in the experimental and control condition varying across a number of dimensions. It is not clear what it is about music and dance that may lead to an increase in cooperation.

I took an alternative approach: isolating a specific behaviour found in nearly all forms of music and dance. If music and dance are pan-human phenomena that arose in the human species because they increased the cooperative tendencies of performers, then we might expect that certain core mechanisms by which music and dance foster cooperation will recur in nearly all cultures. Despite its variance, all forms of group music and dance involve people matching rhythmic behaviours in time (Lomax, 1968) - or what I call synchrony making it a prime candidate for a potential cooperative mechanism. It is the effect of group synchrony on cooperation which was the specific focus of this thesis. I isolated synchrony's effects by minimising other social, cultural, and emotion factors usually present in group ritualised music and dance.

Reducing music and dance to synchrony has the benefit of easy manipulation: participants can be instructed to move rhythmically in time with each other and the effects of this can be compared against a condition in which participants are instructed to move out of time with each other. It also provides a cross-cultural mechanism by which music and dance could foster cooperation. If synchrony, even under the minimal and controlled conditions of a laboratory, produces cooperative effects it could suggest that what specifically is sung and what specific actions are performed in a particular cultural manifestation of music and dance 
are not of prime importance in explaining the universality and retention of music and dance in the human species ${ }^{1}$. Rather, what is essential is that people are performing in time with each other. Furthermore, focusing on synchrony links music and dance to an expanding literature on social embodiment - the discovery that how human's interact their bodies with each other, particularly in form (e.g., mimicry - the non-conscious imitation of another's actions) and time (e.g., synchrony), influences our social cognition and behaviour (Bernieri \& Rosenthal, 1991; Chartrand \& van Baaren, 2009; Hove \& Risen, 2009; Semin \& Cacioppo, 2008; Stel, Van Baaren, \& Vonk, 2008; Valdesolo \& DeSteno, 2011). Such studies have concentrated on the interaction of pairs of people (dyads) looking to explain interpersonal bonding. For this thesis, I applied the methods and results of these dyadic mimicry and synchrony studies to the study of group behaviour expressed in music and dance.

Of course, the limitation of such control is ecological validity. For music and dance in the real world is not simply moving in time - it involves a variety of social, cultural, emotional, cognitive, and motivational factors. However, the scientific approach works by slow accumulation. The studies incorporated in this thesis serve as a starting block for exploring the cooperative effects of music and dance under the most minimal conditions. Future studies can build on the findings contained herein to examine the effects and interaction with other key variables involved in music and dance.

Operationalising cooperation also provides some challenges. Cooperation generally refers to behaviours that directly benefit another individual. However, this definition is somewhat vague as both behaviour and benefit incorporate a wide range of possibilities. In evolutionary models the benefit refers to an increase in reproductive success (Stevens, Cushman, \& Hauser, 2005; West, Griffin, \& Gardner, 2007). Measuring benefit in this way is obviously not practical for an experiment at a single point in time. Social psychology has examined cooperation under the term prosocial behaviour (Batson, 1998), where the benefit is usually the direct voluntary giving of resources to another person whether monetary, material, or time, often at a cost to the giver (e.g., North, Tarrant, \& Hargreaves, 2004; Wilson, Tumminelli, Brien, \& Sesma, 2009; van Baaren, Holland, Kawakami, \& van Knippenberg, 2004). Another line of research has investigated cooperation in humans through simplified models which are experimentally tested with economic games (e.g., Haley \& Fessler, 2005; Ruffle \& Sosis, 2007; Shariff \& Norenzayan, 2007). Such games are usually

\footnotetext{
${ }^{1}$ That is not to say that the particular words and actions in a song or dance have no effect. Certainly some experimental data suggests what is sung can affect the level of cooperation (Greitemeyer, 2009). However, such specificity is unlikely to be a major driving force behind the development of music and dance in the human species.
} 
contrived situations where participants are given specific rules for the allocation of money between people. Participants are often put in a dilemma about whether to keep money for themselves or allocate it to others or a shared pool. The amount of money allocated to another or allocated to a shared pool is usually taken as a measure of cooperation - that is how much they are willing to benefit another, often at a potential cost to themselves. To explore the cooperative effects of synchrony, I used both social psychological helping measures as well as economic games.

\section{Why might synchrony increase cooperation?}

As well as examining if group synchrony fosters cooperation, this thesis also aims to further understanding of how synchrony may produce such an effect. Elucidating the causal mechanism of any psychological process is a thorny endeavour. A mechanism can be examined from multiple levels (e.g., from neurons to societies) and could involve multiple interacting processes (e.g., emotional, cognitive, social). A full explanation of the mechanism for how synchrony could produce greater cooperation is beyond the scope of the thesis. Instead, I examined a few key mediating psychological variables that may be able to shed light on potential causal paths. Based on previous theorising and empirical research, I focused in particular on perceived similarity, entitativity, and interdependent self-construal. As well as providing theoretical models for how these psychological constructs could mediate the relationship between synchrony and cooperation, I also measured these constructs using selfreport questionnaires.

\section{Outline of the thesis}

This thesis is composed of six chapters and five laboratory-based social psychology experiments. In chapter 2, I define synchrony and introduce the concept of entrainment. Synchrony, as I use the term, is not just limited to music and dance, but occurs in many human interactions. The aim of chapter 2 is to integrate music and dance into this broader framework of synchrony. I outline several plausible proximal and ultimate explanations for why synchrony is performed by humans. This then leads to chapter 3 in which I detail the theory behind the music/dance-cooperation hypothesis and the current evidence in support of it. 
In Chapter 4, I describe three experiments that examined if behaving in synchrony with others under conditions in which emotional, social, and cultural factors are minimised, was sufficient to increase cooperation. All three experiments manipulated synchrony in a similar way: participants in small groups of three or four were asked to perform specific movements or speak out loud specific words in time with a metronome beat. In one condition, participants heard the same metronome beat resulting in synchronous movement. In another condition, participants heard metronome beats that were at different tempos resulting in asynchronous movement. An additional control in which participants were passive and did not move or vocalise was also used. The three experiments varied in the behaviour participants were asked to do, the way cooperation was measured, and who the cooperation was targeted towards. Experiment 1 examined synchronised movement and measured the level of cooperation directed towards both fellow performers and nonperformers with a natural helping scenario. Experiment 2 examined synchronised vocalising and measured the level of cooperation directed towards both fellow performers and nonperformers with two different natural helping scenarios. Experiment 3 examined synchronised movement (as in experiment 1), but measured cooperation with an economic game directed at fellow performers.

In chapter 5, I examined if I could obtain larger and more robust increases in cooperation by creating synchrony with shared intentionality. I initially tested this hypothesis by running a new condition after experiment 3 in which participants worked together to create synchrony (instead of synchrony being created incidentally by having the same metronome beat). In experiment 4, I compared the cooperative effects of synchronised chanting created through shared intentionality to sequential chanting created through shared intentionality. In experiment 5, I manipulated both synchrony and shared intentionality to examine the interaction between these two variables.

In the final chapter I discuss the evidence these five experiments provided in answering the two key questions of this thesis: does group synchrony increase cooperation, and if so, through what mechanism does it occur. I then assess how well these results can be generalised to music and dance as found in natural human ecologies and if the results can help inform why music and dance exists in the human species. 


\section{Chapter 2}

\section{INTERACTIONAL SYNCHRONY IN HUMANS}

\section{What is synchrony?}

Objects that move rhythmically (oscillators) often do not operate in isolation but their rhythms interact with other oscillators resulting in the rhythms falling into a constant relationship with each other (Glass \& Mackey, 1988). This interaction process is termed entrainment (Clayton, Sager, \& Will, 2004). Synchrony is the term used to describe the state when two rhythms have become tightly entrained. Entrainment can involve both the matching of frequency and the matching of phase (Clayton et al., 2004). A wider definition of synchrony includes any situation in which rhythms are frequency entrained (frequency-locked synchrony) resulting in the rhythms sharing the same frequency or some simple ratio of frequencies (e.g., one rhythm is twice the speed of another rhythm). A narrower definition of synchrony only includes cases of entrainment where the rhythms are exactly in-phase with each other (phase-locked synchrony). Phase-locked rhythms are also frequency-locked, but frequency-locked rhythms are not necessarily phase-locked. When unspecified, the term synchrony as used below refers to the more inclusive category of frequency-locked synchrony.

As a general process, entrainment is very common and can be seen in both biological and non-biological oscillators, over different periodicities, and at different levels of the universe. For example entrainment has been observed with sub-atomic particles (e.g., lasers), planets (e.g., the moon to the earth), cells (e.g., as in the heart), and organisms (e.g., fireflies), (see Strogatz, 2003 for a review). Entrainment occurs through communication of rhythmic information (Zivotofsky \& Hausdorff, 2007). In non-living oscillators, entrainment is incidental and caused by physical forces produced by a rhythmic oscillator directly affecting the rhythmic movement of another oscillator. For example, with two pendulum clocks moving at different speeds but attached to the same wall (where the process of entrainment was first noted by Huygens in 1665), the cyclical force produced by a pendulum clock travels through the wall, shaking the other pendulum clock at its rhythm (Bennett, Schatz, Rockwood, \& Wiesenfeld, 2002). That pendulum clock likewise sends forces through the wall shaking the other pendulum clock. Eventually they both end up moving at the same 
speed. In biological entities, the entrainment process between organisms can be much more complicated as there often is not a direct physical force between oscillators. Entrainment may instead occur through chemical processes (Massie, Blasius, Weithoff, Gaedke, \& Fussmann, 2010) or through sensory systems (Phillips-Silver, Aktipis, \& Bryant, 2010). Entrainment in organisms appears to serve an important function: it helps in the temporal coordination of the organism to its environment and other organisms so as to aid in survival and reproduction. For example, timing is often critical in finding fertile mates, appropriate food supplies, or avoidance of predators (King \& Cowlishaw, 2009).

The entrainment between two or more organisms is called social entrainment (Phillips-Silver et al., 2010). In humans, social entrainment can occur with a variety of different rhythms of varying periodicities (Hayes \& Cobb, 1982; McGrath \& Kelly, 1986). Of particular interest for this dissertation is entrainment of behaviours that occurs during the time frame of a social interaction - that is interactional synchrony. It is entrainment of such behaviours that I will be referring to when I use the term synchrony from now on.

\section{Interactional synchrony}

Human movement and speech has natural rhythmic qualities to it (Cummins, 2009; Gallistel, 1980; Newtson, Hairfield, Bloomingdale, \& Cutino, 1987). During an interaction, people are able to perceive these rhythmic elements in others and match their own behaviour to it. Humans are quite flexible in their capacity to entrain to others' rhythms. People can perceive rhythms from a wide variety of different behaviours, of varying complexity (Large $\&$ Palmer, 2002), varying frequencies (from around $0.5 \mathrm{~Hz}-10 \mathrm{~Hz}$; London, 2004), and through a number of sensory modalities: visual (e.g., Richardson, Marsh, \& Schmidt, 2005), auditory (e.g., Condon \& Sander, 1974), tactile (e.g., Zivotofsky \& Hausdorff, 2007), and vestibular (e.g., Phillips-Silver \& Trainor, 2008; Trainor, Gao, Lei, Lehtovaara, \& Harris, 2009). People can then entrain their own behaviour with an infinite variety of expressions involving both similar muscle groups (e.g., entrainment of the speech rhythms to the speech rhythms of another; Cummins, 2009) or with very different muscle groups (e.g., entrainment of body movements to the speech rhythms of another; Shockley, Santana, \& Fowler, 2003). People can entrain at various ratios of the perceived beat (Parncutt, 1994) and also various phase relationships - although in-phase and anti-phase (that is $180^{\circ}$ out of phase) are the easiest (Schmidt, Carello, \& Turvey, 1990). The key proposed mechanism to allow for entrainment is the integration of rhythmic information gathered through sensory systems with rhythmic 
information generated in the motor production systems through some kind of neural feedback (Phillips-Silver et al., 2010; Todd, Lee, \& O’Boyle, 2002).

\section{Unintentional synchrony}

Humans both intentionally and unintentionally synchronise their behaviours during social interactions (Oullier \& Kelso, 2009; Richardson et al., 2005). ${ }^{2}$ That is, sometimes people have an explicit goal of trying to match behavioural rhythms and sometimes people fall into synchrony spontaneously without realising it. Unintentional synchrony is subtle and can be difficult to observe. Early studies on unintentional synchrony were investigated using videos of natural social interactions. Condon and Ogston (1967) used a microanalysis technique where they examined films of adult conversations frame by frame. They found that changes in movement from a listener often accompanied rhythmic changes in speech pattern from the talker. Other authors also found similar results with movement matched to speech in both adults (Dittmann \& Llewellyn, 1969; Hadar, Steiner, \& Rose, 1985; Kendon, 1970; Rutter \& Stephenson, 1977) and infants synchronising to adult speech (Berghout-Austin \& Peery, 1983; Kato et al., 1983). Unintentional synchrony has been found with speech convergence (Street, 1984), conversation pausing frequency (Cappella \& Planalp, 1981), postural sway (Shockley, Baker, Richardson, \& Fowler, 2007; Shockley et al., 2003), and walking (Van Ulzen, Lamoth, Daffertshofer, Semin, \& Beek, 2008; Zivotofsky \& Hausdorff, 2007). People also rate 'real' interactions as more synchronous than 'pseudo-interactions' where videos of two people from different time points in an interaction are merged together (Bernieri, 1988; Bernieri, Davis, Rosenthal, \& Knee, 1994).

Unintentional interpersonal synchrony has also been examined in more contrived situations. Schmidt and O'Brien (1997) asked participants to swing pendulums at the speed that they were most comfortable with. Participant's cross-spectral correlation of rhythm frequencies was greater when participants were viewing each other than when no information was shared with phase relationships predominantly being in-phase or anti-phase. Richardson et al. (2005) replicated this result and also found that the presence of conversation did not affect the level of synchrony. Likewise, Richardson, Marsh, Isenhower, Goodman, and Schmidt (2007) found the same effect with rocking chairs even when the natural periodicities of the chairs were different. Notably, the participants were unaware that they had altered their

\footnotetext{
${ }^{2}$ Knoblich, Butterfill, and Sebanz (2011) used a similar differentiation for interpersonal coordination using the terms emergent coordination and planned coordination.
} 
tempos. These results suggest that unintentional synchrony can occur with simple visual coupling even in the absence of joint conversation.

\section{Intentional synchrony}

With intentional synchrony, a person plans to synchronise their behaviour with another (Knoblich \& Sebanz, 2008), attends to the rhythmic stimulus, and has some degree of awareness they are synchronising. Whereas unintentional synchrony appears to predominantly occur in dyads with simple body rhythms, intentional synchrony is more flexible and can be generated from more complex rhythmic behaviours and commonly occurs in groups (Knoblich, Butterfill, \& Sebanz, 2011). Intentional synchrony is used in joint action tasks: "any form of social interaction whereby two or more individuals coordinate their actions in space and time to bring about a change in the environment" (Sebanz, Bekkering, \& Knoblich, 2006, p. 70). Such coordinated action often involves shared intentionality (Tomasello et al., 2005) - those involved in the interaction share a goal, share an action plan as to how to complete that goal, and shared attention directed at the unfolding joint behaviour. Deliberately creating synchrony often aids in completing a functional goal. For example, in rowing a boat the goal is to move across water in the desired direction. Being in synchrony helps this happen faster and more efficiently. Intentional synchrony also occurs where the entrainment of rhythms between two or more people is itself the primary goal of the behaviour. Such behaviour we usually term music or dance.

\section{Synchrony in music and dance}

Music and dance are terms that incorporate a cluster of behaviours in which certain shared characteristics may or may not be present in any particular cultural expression (i.e., it is a polythetic category) and where certain defining characteristics are often on a continuum (i.e., it is a fuzzy set), (c.f. Snoek, 2006). As discussed in chapter 1, this has led to debate about definitions for music and dance (Bispham, 2006; I. Cross, 2001; Hanna, 1987). In this thesis, I define music and dance as the intentional social entrainment of rhythmic sound or body movements for which the creation of synchrony is divorced from any direct functional utility for being in time. ${ }^{3}$ This definition separates music and dance from other forms of

\footnotetext{
${ }^{3}$ Cross (2003) used a similar definition for music emphasising entrainment, embodiment and intentionality.
} 
intentional synchrony in that the creation of synchrony is not the means but the ends of the activity (Schachner \& Carey, 2011). Synchrony is performed because it is desirable in and of itself. There is no obvious external goal - or at least any proclaimed external goal is not causally related to the action of being in synchrony (I. Cross, 2001). Such ostensible nonfunctional behaviour divorced from any practical goal has been highlighted as a key aspect of ritual (Boyer \& Lienard, 2006; Rappaport, 1979). Indeed music and dance often takes place in what are commonly described as religious rituals and may play an important role in the function of rituals (Durkheim, 1965; Nettl, 1983; Rust, 1996).

In defining music and dance in this way, I include some behaviours that may not usually be termed music or dance in English speaking cultures - such as group chanting and marching. This definition also leaves out some behaviours that are commonly termed music and dance, in particular individual musical expressions. However, in most cultures around the world and through most of our history, music and dance have been social phenomena - the product of social entrainment (Nettl, 1983; P. Spencer, 1985). In support of music's social nature, research by Kirschner and Tomasello (2009) has found that young children are more motivated and more accurate at entraining to a beat with another person rather than a drumming machine or a beat coming through a speaker. This result shows that from a young age, children have the ability and motivation to pursue music as a social activity.

In most cultures music and dance are tightly linked (Arom, 1991; Blacking, 1995; Merriam, 1964), with some cultures not having separate words for music and dance (Gourlay, 1984). In both psychology and anthropology, research has predominantly focussed on music rather than on dance. However, in both disciplines, an increasing number of authors are emphasising their inter-relation (Bellah, 2003; I. Cross, 1999, 2001; Freeman, 2000; Gorer, 1972). For example Lange (1975) states:

A good deal of music of all kinds developed out of dancing activities. The influence of dance on the shaping of music forms is also evident. Musicologists could have solved many problems concerning some facts on music origins if there had existed concurrently a wider knowledge of dance. (p. 103)

Evidence for the music-dance link has also been found through recent experimental work that suggests we have tightly coupled auditory-motor systems (Repp \& Penel, 2004; Zatorre, Chen, \& Penhune, 2007). Certainly anecdotal observation suggests the ease and automaticity of motor movements such as foot or finger tapping when we hear music. This motivation to move our bodies to music occurs from a very young age. Zentner and Eerola 
(2010) found that infants (5-24 months) were more likely to move rhythmically to music (including simple drum beats) than speech. Moreover, the degree of coordination was positively correlated to displays of positive emotion, suggesting that as well as being motivated to dance to music we enjoy creating synchrony.

Although music and dance both involve the entrainment of rhythmic behaviours, the actual behaviours performed and the context they occur in are highly varied across cultures and even within a culture. Music and dance vary across such factors as the number of people who take part, who specifically can take part, the use of musical instruments, what instruments are used, the speed of the rhythm, the meaning behind the activity, what specifically is sung about, when the activity takes place, where the activity takes place, how long it lasts for and so on.

The most comprehensive analysis of this variance in musical and dance expressions comes from Lomax and collaborators (Lomax, 1968) who developed cantometrics and choreometrics, which are the systematic analysis of song style and dance style respectively from around the world (around 4000 songs from 400 cultures, Lomax \& Berkowit, 1972). Important to the present study, Lomax (1968) found diversity in the level of synchrony and integration in the performance. Some performances involve everyone singing exactly the same tone and words at exactly the same time (phase-locked synchrony), other performances involve different subgroups singing different words or different tones, some performances involve individuals singing different melodies, other group performances also include a soloist. Of course singing is usually accompanied by instrumentalists who may be playing different instruments and different rhythms or melodies. Despite this variance, a shared beat for which individuals socially entrain to underlies all.

\section{Why does interactional synchrony occur?}

To understand why a behaviour occurs, we need to distinguish between proximate and ultimate causes. Proximal causes are those biological, psychological and social factors that lead an individual to perform a specific behaviour. Ultimate explanations examine why a behaviour arose in a species. 
Coordination aid for collaborative activities

Human's ability to entrain to other people's behavioural rhythms aids an individual by helping them coordinate their behaviour with others, what Vesper, Butterfill, Knoblich, and Sebanz (2010) termed coordination smoothers. The inherent repetitiveness of rhythms means that they are useful to predict behaviour (Repp, 2005). The better one can predict behaviour, the better one can coordinate (Vesper, van der Wel, Knoblich, \& Sebanz, 2011). One theory for why unintentional synchrony occurs during conversations, is that the entrainment to the verbal rhythms of another aids in the coordination of a conversation (M. Wilson \& Wilson, 2005). We often do not notice the ease with which we verbally interact until we are in a situation where the timing has been disrupted such as a delay on a long distant phone call (Bernieri \& Rosenthal, 1991). Some of the unintentional synchrony that occurs between a listener's body movements and a speaker's verbal rhythms may also serve a similar functional purpose, helping to maintain an interaction when verbal synchrony breaks down (Rutter \& Stephenson, 1977). Intentional synchrony can also perform a similar function with more complex joint action tasks (Sebanz \& Knoblich, 2009). Two or more people coordinating their movements in time can extend the range of actions that just one person could do by themselves.

\section{To build affiliation}

Another proximal function that has been suggested is that unintentional synchrony is used (non-consciously) as a tool to build affiliation and establish rapport (Kendon, 1970). A couple of studies on natural social interactions have found significant positive correlations between the degree of synchrony and rapport in natural conversations (Bernieri, 1988; Warner, Malloy, Schneider, Knoth, \& Wilder, 1987). Tickle-Degnen and Rosenthal (1990) even argued that interpersonal coordination, including synchrony, is a fundamental aspect of rapport. They argue for a tripartite model of rapport incorporating coordination (feelings of balance and harmony) along with attention (feelings of mutual interest and focus) and positivity (feelings of friendliness and caring), with feelings of balance and harmony in an interaction being correlated with interpersonal synchronous behaviour. 
Further evidence that synchrony may be used to help establish rapport comes from experimental studies. Miles, Griffiths, Richardson, and Macrae (2010) found participants unintentionally synchronised with an interaction partner (a confederate) when that partner was on time. But synchronisation was not above chance levels if the interaction partner was late. Furthermore, the degree of rapport participants anticipated with their interaction partner predicted the level of synchrony. In another study, Miles, Lumsden, Richardson, and Macrae (2011) found that greater synchrony was present when interacting with a participant of a different minimal group. The authors suggest that this could be due to a desire to affiliate and reduce intergroup differences.

A few studies have also manipulated how in time people actually are with each other. Hove and Risen (2009) used a finger tapping paradigm to examine the social effects of synchrony. In their experiment a participant tapped on a drum machine pad in time with a moving target on a computer screen. In the synchrony condition, the experimenter sat next to the participant and also tapped on another of the drum machine's pads whilst keeping in time with a target on their computer screen that was at the same tempo. Participants were therefore moving at the same frequency and in-phase with each other. In the asynchrony condition, the target on the experimenter's computer screen was at a different rhythmic speed, therefore they moved at different frequencies with each other. In the alone condition, only the participant tapped - the experimenter sat with her hands in her lap. The authors found that participants in the synchrony condition liked the experimenter significantly more than in the asynchrony and alone conditions (measured on a 9-point Likert scale to the question "how likeable was the experimenter"). As it may be the sensation of being in synchrony rather than interpersonal synchrony per se that had the effect, the authors replicated this experiment with a metronome that sounded either in time or out of time with the participants tapping (or was silent in a third condition) while the experimenter sat next to the participant. In this experiment there was no difference in liking of the experimenter. This suggests it is interpersonal synchrony rather than the sensation of being in synchrony that had the effect.

Using a similar finger tapping paradigm, Valdesolo and DeSteno (2011) also found an increase in liking of an interaction partner after synchrony. Moreover, the authors examined if synchrony not only resulted in more felt attraction towards the other person, but actually produced greater prosocial behaviour towards them as well. After the synchrony manipulation participants were placed in a scenario in which they witnessed their interaction partner being unfairly treated and participants were given an opportunity to help. Participants who moved in synchrony were more willing to help and spent longer helping their interaction 
partner than participants who had moved out of synchrony. This relationship between synchrony and helping was mediated by feelings of similarity and compassion towards their interaction partner.

Similar social effects have also been found for mimicry - the unintentional matching of behaviours in form (Chartrand \& Bargh, 1999). Both correlational studies (Charney, 1966; LaFrance, 1979; LaFrance \& Broadbent, 1976; Scheflen, 1964) and experimental studies (Bavelas, Black, Lemery, Macinnis, \& Mullett, 1986; Chartrand \& Bargh, 1999; Maurer \& Tindall, 1983; Stel \& Vonk, 2010) have found a link between greater mimicry and greater levels of rapport and liking directed towards the interaction partner. Mimicry has also been found to have important consequences on social behaviour - people are more helpful, generous, and willing to be closer to another after being mimicked (Stel et al., 2008; Van Baaren, Holland, Steenaert, \& van Knippenberg, 2003). Interestingly, this effect is not restricted to the interacting dyad: people are also more likely to help a complete stranger (Ashton-James, van Baaren, Chartrand, Decety, \& Karremans, 2007; Van Baaren, Holland, et al., 2004).

As with interpersonal synchrony, mimicry has been suggested to be used as a tool for affiliation (Lakin, Jefferis, Cheng, \& Chartrand, 2003). In support of this hypothesis, Lakin and Chartrand (2003) found that more mimicking occurred when there was an affiliation goal. The authors found this effect both when affiliation was explicitly requested and when it was implicitly primed. An increase was also found after a recent failure to affiliate if affiliation had been primed. Further evidence for mimicry being used for affiliation comes from Lakin, Chartrand, and Arkin (2008) who found that participants who were made to feel excluded by their in-group mimicked fellow in-group members more than out-group members. Similarly, Over and Carpenter (2009) found that priming ostracism led to more mimicry. People may also use mimicry to initiate romantic relationships. Mimicry, as well as increasing liking, also appears to increase sexual attractiveness (Gueguen, 2009), and people who are single mimic an attractive opposite sex more than people involved in a romantic relationship (Karremans \& Verwijmeren, 2008).

To summarise, results from dyadic synchrony and mimicry studies indicate that the way we move our bodies in relation to others seems to have an important effect on our social cognition (Barsalou, Niedenthal, Barbey, \& Ruppert, 2003; Marsh, Richardson, \& Schmidt, 2009; Semin \& Cacioppo, 2008). People may subconsciously learn that modifying one's actions to be similar or to be entrained to another's is a way of building interpersonal relations. 
Despite music and dance involving similar underlying behaviour to other forms of interactional synchrony, music and dance have been argued to serve a much wider variety of purposes for both the individual and the social group. Merriam (1964) proposed ten key proximal functions that music around the world fulfils: emotional expression, aesthetic enjoyment, entertainment, communication, symbolic representation, physical response, enforcing conformity to social norms, validation of social institutions and religious rituals, contribution to the continuity and stability of culture, and contribution to the integration of society. Levitin (2008), proposed six functions for music: to facilitate social bonding in groups, to express joy, to comfort us, to help transmit knowledge, to facilitate religious rituals, and to communicate love. Clayton (2009) suggested four functions: regulation of an individual's emotional, cognitive, or physiological state; mediation between self and others; symbolic representation; and coordination of action. A number of functions have also been described for music listening based on cross-cultural data (Boer et al., 2012; Boer \& Fischer, 2012). Although these lists concentrate on music, each author does integrate dance but more as a function for music rather than dance having its own specific function. Multiple functions for dance have been discussed by dance anthropologists. Spencer (1985) highlights the following functions for dance: catharsis or expression of emotion, education, convergence of emotions and societal values, social bonding, pleasure, to take people out of normal societal structure, and as a boundary display against opposition groups. Other functions such as healing (L. Marshall, 1969), aesthetic appeal (Boas, 1972), religious ritual (Lange, 1975), and communication (Hanna, 1987) have also been proposed.

Such a variety of functions for music and dance suggest that music and dance may have originated from a general propensity to be able to move in time with others, with various individuals and cultures utilising this ability to fulfil their needs. However, a number of academics have argued that music and dance may in fact be an adaptation: it was selected through some combination of biological and cultural evolution because it provided important survival and reproductive advantages to those who practiced it. It is to the ultimate explanation of interactional synchrony and in particular music and dance that I now turn. 


\section{Ultimate causes for interactional synchrony}

Social entrainment has not yet been observed in human's closest phylogenetic relatives the chimpanzee (or any of the great apes, Fitch, 2006; Schachner, 2010a). Groups of chimpanzees have been observed rhythmically beating their bodies and trees, and vocalising loudly, but no synchronisation appears to occur (see Powers, 2005, for a review). This could suggest that homo sapiens developed this tendency to socially entrain after diverging from a common ancestor of humans and chimpanzees. The finding that interpersonal synchrony occurs automatically in response to mere observation of others moving rhythmically (e.g., Richardson et al., 2007, 2005; Schmidt \& O’Brien, 1997) suggests that humans may have evolved neural algorithms linking rhythmic information (within the possible frequencies of human movement) gathered through the senses to motor production systems. What selection pressures may have led to the evolution of such a system? One possibility is that the ability to entrain with others may have arisen in the human species due to the benefits of being able to effectively coordinate with others would have brought to our ancestors. Shared intentionality has previously been argued to have greatly extended human's cooperative ability and to have been important in the creation of human culture (Tomasello et al., 2005). Although sharing mental states to initiate joint action is a vital component, being able to effectively complete joint action by meshing one's behaviour with another's with careful timing is also critical (Sebanz et al., 2006). Such a general function is supported by the flexibility of the entrainment system to be able to integrate a variety of rhythmic information from different senses into the execution of infinite rhythmic muscle patterns.

Our social entrainment ability may have also undergone further evolution in the creation of language. Being able to move muscles involved in vocalisation with precise timing so as to replicate sounds produced by others would have been an essential evolutionary development in the creation of language (Patel, 2006). The adaptive benefits of being able to effectively communicate through language could have resulted in human's strong neural auditory-motor links (Zatorre et al., 2007) and could explain why we are better at entraining to auditory rhythms than visual rhythms (Repp \& Penel, 2004). Evidence for the link between vocal learning and entrainment has come from comparative studies. Patel, Iversen, Bregman, and Schuiz (2009) describe a domesticated sulphur crested cockatoo - a species renowned for its vocal mimicry ability - that would spontaneously bob its head and move its feet rhythmically in time with music. When the experimenters changed the tempo of the music, the cockatoo adjusted the speed of its movements to stay entrained to the beat. 
Further comparative evidence for the vocal-learning-rhythmic-synchronisation hypothesis comes from Schachner, Brady, Pepperberg, and Hauser (2009). The authors analysed videos taken from around the world of a variety of animal species moving rhythmically to human produced music. They found that the ability to entrain was only present in those species that were also capable of vocal mimicry - 14 species of parrot and the Asian elephant. However, all the animals analysed were domesticated and had a history of interactions with humans. Although it provides evidence that these species have the ability to entrain, whether these species naturally entrain their movements to external rhythms remains to be discovered. These animals also only entrained to an external beat - not another member of their species. Again, it remains uncertain whether they have the ability to socially entrain.

Whatever selection pressures may have led to the creation of our ability to socially entrain, it seems a reasonable conjecture that this ability evolved before music and dance. Social entrainment appears to be a general ability seen across a variety of human behaviours. Music and dance, on the other hand, is a specific form of this general ability. Music and dance, therefore, may not be a product of evolutionary selection, but a by-product of other evolved abilities. Pinker (1997), in particular, supports this view claiming that music is: "pure pleasure technology, a cocktail of recreational drugs that we ingest through the ear to stimulate a mass of pleasure circuits at once" (p. 528). Humans designed music to tap into other evolved adaptations that give us pleasure. Pinker's view of music may be valid for modern recorded music for which the life of a song is dependent on how well it tickles people's fancy, but in terms of explaining music and dance in the human species, Pinker's hedonistic by-product theory has been critiqued as being ethnocentric (I. Cross, 2001). In particular, Pinker does not take into account the social nature of music and dance as expressed in most cultures and music's close connection with dance. An alternative view, now gaining in popularity, is that music and dance may have undergone evolutionary selection and become an adaptation due to specific adaptive benefits that performing music and dance produces (Bispham, 2010; Cross, 1999, 2001, 2003, 2009; Hauser \& McDermott, 2003; Huron, 2001; Mithen, 2005; see Wallin, Merker, \& Brown, 2000).

There are a number of elements about music and dance that suggest it could have the hallmarks of an adaptation. As previously mentioned, music and dance have a long history in the human species. Flutes found in Europe date from approximately 40,000 years ago (Conard et al., 2009). However, it is likely that music considerably predates this as the flutes found are highly complex with the earliest music traditions likely employing vocalising or simple percussive instruments made from biodegradable or unmanipulated material (Adler, 
2009). Similarly, cave drawings of dance suggest a history older than 9,000 years (Chakravarty and Bednarik, 1997; cited in Schachner, 2010b). This long history could have provided time for biological and/or cultural evolution to have operated. Furthermore, music and dance are ubiquitous, being found in all cultures and with the vast majority of people partaking in music and dance. Given that music and dance involves the expenditure of large amounts of energy (Huron, 2001) and so in this respect incurs a cost, it would seem unlikely that music and dance would have existed for so long and have spread throughout the world if it did not also produce some important benefit.

If music and dance was an adaption, what function might it perform to have led to a selective advantage for those who practice it? As music and dance is separated from any ostensible functional goal, the answer has not been obvious. One method to explore this question has been to examine potential analogous behaviour in other species. Although entrainment as a general process is very common throughout the animal kingdom, social entrainment of rhythmic interaction behaviours, especially in groups, appears to be rare. Gibbons, along with a number of bird species, do perform tightly coordinated dyadic vocalising known as duetting (Geissmann, 2000; Mann, Dingess, \& Slater, 2006; Wickler, 1976). Such behaviour is thought to be involved in territory signalling and is associated with long-term bonded pairs. Hagen and Bryant (2003) suggested that music and dance may have evolved to perform a similar function: to defend group territories. Music and dance might very well be used for such a display function for other groups, but as Hagan and Bryant themselves note, music and dance often occurs when other groups are not present. Such a theory then would only explain a small proportion of group music and dance performances.

Using the vocal courtship displays of animals as an analogy, Darwin (1871) and later Miller (2000) suggested music may have evolved through sexual selection to attract members of the opposite sex. Although courtship displays may involve the production of rhythmic sound, most courtship displays lack social entrainment. Synchronised group displays of courtship displays have been observed in fireflies, fiddler crabs, katydid, and a few frog species (see Greenfield, 1994, for a review). In these cases, the synchronisation between males is thought to aid in the attraction of females to the group (Backwell, Jennions, Passmore, \& Christy, 1998; Moiseff \& Copeland, 2010). Merker (2000) argues that this function of attracting females to a group could explain group music and dance in humans. However, in both these cases the analogy is probably not apt: courtship displays are generally restricted to the male of the species and of reproduction age. Music and dance in humans, on 
the other hand, are performed by both sexes, of all ages, and in most expressions around the world play no direct role in courtship (see Brown, 2000, for a critique of these theories).

The most popular explanation for the retention of music and dance in human culture is that it increases cooperation within the group resulting in a better functioning group that is more likely to survive and reproduce (Adler, 2009; Bellah, 2003; Benzon, 2001; Bispham, 2010; S. Brown, 2000; Cohen, Ejsmond-Frey, Knight, \& Dunbar, 2010; I. Cross, 2001; Ehrenreich, 2006; Freeman, 2000; Haidt, Seder, \& Kesebir, 2008; Huron, 2001; Kirschner \& Tomasello, 2010; Levitin, 2008; McNeill, 1995; Mithen, 2005; Peretz, 2006; Roederer, 1984; Wiltermuth \& Heath, 2009). This theory I term the music/dance-cooperation hypothesis. In the next chapter I present the logic behind this theory and the evidence that currently exists to support it. 


\section{Chapter 3}

\section{THE MUSIC/DANCE-COOPERATION HYPOTHESIS}

\section{Theoretical overview}

Living in groups is highly adaptive. It increases the survival and reproduction of those in the group by helping in the acquisition of resources, defence against predators and enemies, finding mates, rearing young, and care when sick or injured (Dunbar \& Shultz, 2010). However, to effectively sustain group living individuals need to cooperate - to behave in ways that also benefit other individuals in the group (Hardin, 1968). A number of mechanisms have been highlighted to account for cooperation seen across species (e.g., kin selection, Hamilton, 1964; reciprocal altruism, Trivers, 1971) and cooperation in humans specifically (e.g., social identity theory, Kramer \& Brewer, 1984; morality, Haidt, 2007). The music/dance-cooperation hypothesis posits that music and dance could be another method that cultural groups use to help facilitate group living by increasing cooperative tendencies in practitioners. If music and dance did fulfil this function, the importance of group living for human survival could have been a strong selective pressure for its retention and spread in human populations.

The music/dance-cooperation hypothesis posits that the adaptive benefit of music and dance is at the group-level, rather than the individual level. Individuals expend the energy to dance or create music together with the result that collectively they are better off for it, with the collective benefit outweighing any individual cost (S. Brown, 2000). Theories on group selection have gone through various transitions (see West, El Mouden, \& Gardner, 2011 for a review), but there is general consensus that multi-level evolutionary models including adaptation at group-level are possible (Maynard Smith \& Szathmary, 1995; Nowak, 2006; D. S. Wilson \& Sober, 1994). Importantly for the evolution of music and dance, Boyd and Richerson (2010) theorise that group selection of certain cultural traits can operate in groups of distantly related individuals. A number of social psychological processes - such as conformity bias and moralistic punishment - can stabilise particular behaviour within a group, with various intergroup processes such as in-group bias increased through symbolic markers limiting the flow of ideas between groups. Selection of certain cultural traits can then occur through such mechanisms as variation in extinction rates (for example war has been shown to 
be an important selection pressure, Bowles, 2009), imitation of successful neighbours, and selective migration (i.e., movement to more desirable societies).

As the specific forms of music and dance are passed on culturally, cultural evolution likely played some role in any adaptive selection of music and dance. However, some researchers have argued that our propensity to sing and dance might be the result of geneculture coevolution. Although the ability to socially entrain seems more likely to have evolved for a more general purpose such as executing joint action tasks, Schachner (2010) suggests that the motivation to dance and make music together could be a biological adaptation. For example, the following hypothetical scenario describes how biological and cultural evolution may have interacted to select for group music and dance behaviours. Groups that included people who enjoyed entraining with each other may have been more likely to have gained the benefit of collaborating on a functional task as well as the additional benefit of bonding with other group members. Groups that entrained together at the same time may have created particularly strong group bonds. As the number of people who enjoyed entraining together grew, eventually people may have wanted to entrain irrespective of the functional goal. Thus group music and dancing was born. In groups originating with more individuals with a propensity to dance, dancing could become the norm through various social psychological mechanisms (as per Boyd and Richardson, 2010) - for example by nondancers being berated for not joining in. ${ }^{4}$ Groups who danced together would then create better group cohesion and cooperation and so outperform other groups. Dancing culture and genes could be passed to other groups by successful groups taking over and interbreeding with other groups, or the successful group might become so large it splits passing on dancing culture and the genetic predisposition for dance loving to the resulting groups. If the proportion of dance haters increases enough so that the equilibrium shifts to a no-dancing culture, the group won't perform as well and so the group will have less chance of survival. As groups with a greater proportion of dance lovers would survive, dance loving would eventually spread through the human population.

\footnotetext{
${ }^{4}$ For example Werner (1984) describes the social pressure present in Mekranoti Indian group singing: "Hounding the men still in their lean-tos [is] one of the favorite diversions of the singers. 'Get out of bed! The Kreen Akrore Indians have already attacked and you're still sleeping,' they [shout] as loudly as they [can].... Sometimes the harassment [is] personal as the singers [yell] out insults at specific men who rarely [show] up." (p. 245-247, cited in Huron, 2001).
} 


\section{Anthropological and historical evidence for the music/dance-cooperation hypothesis}

Although an ultimate function for music and dance increasing group cooperation is theoretically plausible, does the evidence support the theory? One line of evidence supporting the music/dance-cooperation hypothesis is ethnographic observation or written descriptions from historical records. Although such observations do not measure cooperation per se, reports of enhanced prosocial sentiments after group music and dance stretch back to the $19^{\text {th }}$ century.

Grosse (1897), who was one of the first to discuss the social bonding of music and dance, cites a few first-hand ethnographic reports discussing the social effects of particular tribal dances: "two tribes desiring to confirm mutual good feeling, dance it together" (Waitz \& Gerland, 1859, cited in Grosse, 1897, p. 229); "the corroborries [indigenous Australian group dances] are usually appointed for the confirmation of peace after the conclusion of wars and disputes" (Lumholtz, 1889, cited in Grosse, 1897, p. 229). Grosse then suggests that social solidarity may be the original purpose of dance in the human species:

During the dance they are in a condition of complete social unification, and the dancing group feels and acts as a single organism. The social significance of the primitive dance lies precisely in this effect of social unification....It introduces order and connection, at least occasionally, into the rambling, fluctuating life of the hunting tribes. (p. 229, emphasis in original)

Similar conclusions were also made by Durkheim (1915/1965) and Ellis (1923) based on their reading of the ethnographic literature:

A collective sentiment cannot express itself collectively except on the condition of observing a certain order permitting co-operation and movements in unison, these gestures and cries naturally tend to become rhythmic and regular; hence come songs and dances. (Durkheim, 1915/1965, p. 216)

The participants in a dance, as all observers of savages [sic] have noted, exhibit a wonderful unison; they are, as it were, fused into a single being stirred by a single impulse. Social unification is thus accomplished. Apart from war, this is the chief factor making for social solidarity in primitive life. (Ellis, 1923, p. 64)

From the 1920s cultural anthropology took a shift away from comparative study to understanding behaviour in the context of a specific culture. A number of anthropologists still noted important social effects from the group musical and dance performances they 
witnessed. An often cited example (e.g., Haidt, Seder, \& Kesebir, 2008; McNeill, 1995; Wiltermuth \& Heath, 2009) is Radcliffe-Brown's (1948) description of the Andaman Islanders dance:

As the dancer loses himself in the dance, as he becomes absorbed in the unified community, he reaches a state of elation... and at the same time, finding himself in complete and ecstatic harmony with all the fellow-members of his community, experiences a great increase in his feelings of amity and attachment towards them. (p. 252)

Similar observations have also been made from a number of disparate other cultures. For example: "The warriors dance and sing at the Incwala so that they do not fight.... When they dance they feel they are one and they can praise each other" (Kuper, 1947, p. 224); "Boundary loss, the submergence of self in the flow" (Hanna, 1977, p. 119); "An organised group dance may become a powerful factor in strengthening social bonds" (Lange, 1975, p. 83); "The group of warriors and initiates assert their unity by dancing together" (Gorer, 1972, p. 39).

Historians, too, have examined the role that music and dance have played in increasing prosocial tendencies (e.g., Ehrenreich, 2006; McNeill, 1995). For example McNeill (1995) describes how both new political and religious movements (e.g., French and American revolutionaries, Ghost dance \& Cargo cult, early Christian churches) would dance to help create group cohesion. McNeill also suggests that group chanting and singing was used to bring together disparate groups (e.g., group rhythmic prostrating at the start of Islam; formation of a more unified Hinduism from a "confused multiplicity of local cults" p. 95). McNeill in particular concentrates on military drill, due in part to his own experiences of military drill in which he felt "a strange sense of personal enlargement; a sort of swelling out" (p. 2). Drill is now the norm in militaries around the word even though the practical benefits of marching in time in battle no longer exist. Why would armies spend so much time expending the energies of troupes in this manner? McNeill suggests that the dominance of European military in the latter half of the last millennium was in part due to the solidarity created by the reintroduction of close-order drill in the late $16^{\text {th }}$ century that "spread across Europe like wildfire" (p. 3) as it allowed for armies of dedicated but poorly paid and disparate soldiers. He suggests that mass muscular movement in time was, and still is, employed as a particularly effective strategy for creating mass solidarity in a number of 
fascist and socialist governments such as Nazi Germany, China, and presently in North Korea. $^{5}$

\section{Limitations of anthropological and historical evidence}

The anthropological and historical data presented above provides evidence of enhanced prosocial tendencies from a wide variety of different collective expressions of music and dance around the world and throughout history. This data is useful in providing ecologically valid data consistent with the hypothesis that music and dance increase cooperation. However, this data has a few important limitations (see Bulbulia \& Reddish, 2012).

Firstly, the examples given above could be a case of cherry-picking - choosing examples that fit a hypothesis, but ignoring those examples that contradict it. Certainly, there are a few examples of collective dances that do end in harmony. In his study of the Azande people of Central Africa, Evans-Pritchard (1928) agrees that "there is also a tendency for the dance to increase goodwill and to produce a feeling of concord" (p. 459). However, he notes that sometimes "such gatherings create dangers disruptive to the unity and concord of the ceremony" (p. 460) such as "slanderous songs, sexual indiscretions, drunkenness, competition" with quarrels often occurring. Lienhardt (1957) describes how Anuak dances sometimes end in violence: "men would cut in on each other during the dance, and latecomers would try to wrest their partners from those who were already dancing. This gave rise to much fighting and many people are said to have been killed" (p. 352). Likewise, Turner, 1964) describes Ndembu night dances which often resulted in "jealous fights and wifebeatings" (p. 256). In all these three cases alcohol was involved along with mixed gender dancing that was not tightly socially regulated. Jealous emotions combined with reduced inhibitions may explain some of the aggression.

These examples highlight the importance of context - the function and effects of any behaviour in a particular culture depend to some degree on local environmental and social factors (Dilley, 2002). Context is likely to be of importance in providing proximal explanations of why a particular individual or culture performs a particular song or dance. The local environment will always have some effect in the development of an individual

\footnotetext{
${ }^{5}$ See this internet link for recent footage (2001) of mass rallies in North Korea:

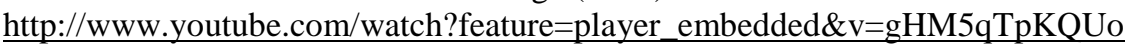


phenotype. However, variation in context is of less importance in ultimate explanations that try and explain general behaviour patterns in a species such as the propensity for humans to move collectively in time. For music and dance to be selected for its cooperative effects, what is essential is that in most cultures in most performances the effect of increased cooperation is produced. As far as I am aware, no study has yet done an in-depth cross-cultural study on the cooperative effects of music and dance performances.

A second limitation of anthropological and historical data is that observations, descriptions, and interviews do not provide precise measurements. Any observation comes with some element of bias and distortion. Ethnographies reduce some of the subjectivity of pure observation by interviewing informants. However, interviews of participants rely to some extent on introspection which has been shown to be an unreliable source for behavioural attribution (Pronin, 2009). People are often not aware why they behave the way do and when prompted give post-hoc rationalisations. To overcome this issue, the construct of interest - cooperation - needs to be operationalised, and more objective measures with good validity and reliability need to be used.

Thirdly, ethnographic methodologies are not designed to establish causation. Even if variables of interest were able to be isolated and objectively measured in the field, without adequate control the causal dynamics is difficult to ascertain. In the field, it is very difficult to control for possible extraneous factors that may have influenced the relationship between a potential causal factor (music and dance) and its effect of interest (increased cooperation). The most effective way to examine causation is with experiments which can compare and evaluate hypotheses by manipulating the variable of interest whilst holding other factors constant and comparing this against a control condition in which the variable of interest in not changed or not present.

\section{Experimental evidence for the music/dance-cooperation hypothesis}

Several experimental studies have been conducted manipulating the presence of music and measuring cooperation. Anshel and Kipper (1988) examined if the presence or absence of music combined with whether participants performed or were passive increased trust and cooperation. Participants in groups of 24 were put into one of four conditions: music participation (singing), music listening, non-music participation (poetry reading), non-music passivity (watching a film). Notably, the poetry reading condition involved participants reading the poem out-loud together in time. Participants then completed a questionnaire on 
how much they trusted the person who sat on their right side, and played a prisoner's dilemma economic game over multiple iterations (30 times) with another randomly allocated group member to test cooperation. In general, the prisoner's dilemma ${ }^{6}$ asks participants to confidentially choose one of two options: (1) a cooperative option $C$ or (2) a defection option $D$. Player one's pay-off in a game (usually money, but in this study points to help become the winner) depends on player two's choice. The maximum payoff for player one occurs if he/she defects (i.e., he/she chooses $D$ ) but player two cooperates (i.e., he/she chooses $C$ ). The next best pay-off is mutual cooperation (both choose $C$ ), then mutual defection (both choose $D$ ), with the lowest payoff for player one being when he/she decides to cooperate when player two defects (i.e., $\mathrm{DC}>\mathrm{CC}>\mathrm{DD}>\mathrm{CD}$ ). Whatever choice player two makes, player one is better off choosing the defection option (i.e., if player two choose cooperate, DC is greater than $\mathrm{CC}$; if player two chooses defect, DD is greater than CD). Defection is therefore the dominant strategy, meaning both players should theoretically converge onto mutual defection, even though mutual cooperation would be better overall. In this study, the number of games out of the 30 that a player chooses the cooperative option was taken to measure their preference for cooperation.

The authors found that self-reported trust was greater in those conditions that involved music, with no difference between whether the music was performed or heard. Moreover, behavioural cooperation (as measured by the economic game) was greater in those conditions that involved participation: singing together in time and reading poetry together in time.

In another more recent study, Kirschner and Tomasello (2010) examined if 4 year old children would be more cooperative with each other after performing a shared musical task compared to children who performed a carefully matched non-musical task. The musical task involved the children singing, dancing, and playing music instruments in time with background music and guided by the experimenter. In the non-musical condition there was no background music, children spoke the lyrics, did not make a sound with the instrument but moved it, and did not dance but walked. Cooperation was measured in two different ways: (1) how helpful a child was in assisting another child who had accidently spilled some marbles; and (2) whether a child chose to cooperate on a task that could be solved either individually or together. For both measures children in the musical condition cooperated more.

\footnotetext{
${ }^{6}$ So called after the metaphor that was originally created to go with the game (see Kollock, 1998).
} 
Both the Anshel and Kipper (1988) and Kirschner and Tomasello (2010) studies provide evidence that performing musically together causes an increase in cooperative behaviour, so supporting the music/dance-cooperation hypothesis. However, it is not clear what specific factor(s) about music might produce these effects. For example, Kirschner and Tomasello's musical and non-musical conditions differed by the presence of a periodic pulse, discrete pitches, a highly repetitive melodic structure, a joint performance context, and expressiveness. Are all of these factors necessary to increase cooperation, or only one or two factors? The finding that dyadic synchrony results in a variety of positive social effects could suggest it is the presence of a periodic pulse allowing for finely tuned socially entrainment that could be critical. To provide stronger support for this hypothesis, group synchrony needs to be manipulated to examine if, isolated from other factors, it leads to greater cooperative behaviour.

\section{Group synchrony and cooperation}

The only paper I have found that experimentally examined the causal link between group synchrony and cooperation was a 2009 article by Wiltermuth and Heath. In their first study, participants walked in groups of three around a university campus either in synchrony or normally. Cooperation was measured with six iterations of the weakest link coordination game. This game followed the basic concept as the prisoner's dilemma, but was different in three key ways. Firstly, mutual cooperation as opposed to defection resulted in the largest pay-out. However, mutual cooperation was also the most risky option - if player one cooperated but player two did not, player one would get the lowest pay-out. This results in two equilibria: (1) a pay-off dominant equilibrium in which a player is attracted to the larger pay-out of mutual cooperation; and (2) a risk-dominant equilibrium in which a player is attracted to the pay-out that will maximise their money irrespective of what the other player decides. Secondly, the choice was not either or, but graded from 1-7: 1 being the most riskdominant choice, 7 being the most pay-off dominant choice. Thirdly, the game was adapted for multiple players, such that a person's pay-out was dependent on the lowest number chosen by a member of the group (hence the name weakest link). The authors found that participants who had walked in synchrony chose higher numbers on average, i.e. they were more willing to cooperate. Participants in the synchrony condition also rated feeling more connected and trusting towards their fellow group members on 7-point Likert scales. 
In their second and third studies, Wiltermuth and Heath investigated the effect of synchronised vocalising as well as synchronised movement. Participants again were placed into groups of three and then took part in one of four conditions. The synchronous singing condition involved participants singing along to a recording of the Canadian national anthem played back through headphones while holding a cup stationary in the air. The Canadian national anthem was chosen "to test whether synchrony can induce cooperation when the soundtrack to the group experience is an out-group anthem" (Wiltermuth \& Heath, 2009, p. 2; their participants were United States residents). Each participant heard the same version at the same time resulting in synchronised singing. The synchronous singing and movement condition was the same as the synchronous singing condition, but participants also waved a cup rhythmically in time with the music played through their headphones. In the asynchronous singing and movement condition, each of the participants in a group heard the Canadian national anthem at a different tempo, resulting in participants moving cups and singing out of time with each other. In the no singing and no movement condition participants simply held the cup in the air and silently read the lyrics to the anthem while listening to the anthem being played through their headphones.

To measure cooperation in the second study, the weakest link coordination game was used again. The authors found that overall participants in both the synchronous conditions were more cooperative than participants in the asynchrony condition and the no singing and no movement condition. No difference was found between both the two synchrony conditions suggesting synchronous singing alone produces a cooperative effect as well as synchronous movement. Participants in the synchronous conditions also rated feeling more on the same team than in the two other conditions.

To measure cooperation in their third study, Wiltermuth and Heath used a public goods economic game. This game is a multi-player version of the prisoner's dilemma in which defection is the dominant strategy: a self-interested participant would do best by defecting, but cooperation would produce the best overall result for the group. Participants were given the opportunity to put 10 tokens in either a group account or a private account. Each token in the group account would earn $\$ 0.25$ for each group member, whereas tokens in the private account would earn $\$ 0.50$ for only that player. Consistent with the results from study two, participants in both synchrony conditions were more cooperative than the asynchrony condition and the no singing and no movement condition. Participants in the synchronous conditions also felt more on the same team than the other two conditions, and felt more similar and trusted marginally more their fellow group members than participants in 
the asynchrony condition. Therefore, Wiltermuth and Heath found data consistent with the hypothesis that group synchrony increases cooperation and replicated this across three different experiments.

\section{Where to go from here?}

Despite Wiltermuth and Heath's promising results, a number of aspects of their manipulation still leave unanswered the specific role that synchrony plays in producing increased cooperation. In their first study, the control condition involved walking normally. However, walking with others often leads to incidental unconscious synchronisation of movement (Zivotofsky \& Hausdorff, 2007). Therefore, the synchrony condition and control condition may not have varied as much in terms of synchrony as anticipated. Moreover, because synchronised walking in public is contra-normative compared to walking normally in a group, the activity may have generated unintended negative affect, which is known to enhance cohesion (Aronson \& Mills, 1959). It could be such shared emotion that bonded the participants together rather or in addition to the synchrony. Although, Wiltermuth and Heath report no difference between conditions in mood, they used a single self-report item ("How happy are you"), which captures pleasant mood rather than emotional arousal (Barrett \& Russell, 1999).

In their second and third experiment these factors were better controlled by asking participants to match their movements and vocalisations in time with music. However, participants were asked to synchronise with a national anthem. National anthems are written to be emotionally arousing, and certainly there is strong evidence for emotional arousal with music (Juslin \& Vastfjall, 2008). Furthermore, singing the anthem of a group that one has a jocular rivalry with may produce humour when several sing in unison. This raises the question of whether prosocial effects are also produced by synchrony with minimal emotional arousal, or if shared emotion is necessary for the effect. Certainly, anthropological theories particularly emphasise the role of shared emotion in ritualised singing and dancing in creating cooperation (e.g., collective effervescence, Durkheim, 1965; communitas, Turner, 1969).

The use of a cultural song could also prime various constructs associated with that song (Seger, Smith, \& Mackie, 2009). A national anthem, for instance, is usually sung together at events where group identity is made very salient (such as before sporting matches). The cultural use of national anthems may have accentuated the participants' own collective identity as Americans. Lyrics of national anthems are also usually written with 
prosocial lyrics and accentuate collective identity. For example $O$ Canada mentions "patriot love" and "we stand on guard for thee". It uses collective pronouns ("we", "our") - there is no "I" or "mine". Although participants sang another country's anthem, it may still have bonded participants together by priming collective identity through such means (Gardner, Gabriel, \& Lee, 1999; Greitemeyer, 2009; Oyserman \& Lee, 2008). The swaying of cups in the air was also probably designed to be similar to the cultural behaviour of swaying alcoholic drinks whilst singing together, again potentially priming collective feelings when performed in synchrony.

To more effectively assess if synchrony is a critical factor in increasing cooperation and to understand how this happens, synchrony needs to be isolated from other emotional, social, and cultural variables. In the next chapter I outline three studies in which I attempted to isolate synchrony by: (1) using a simple metronome beat instead of a cultural song; (2) asking participants to perform simple movements or vocalise simple emotionally neutral words not linked to any particular cultural ritual; and (3) examining synchronised movement and synchronised vocalising separately. Isolating synchrony in this way could also aid in discovering how behaving in synchrony might increase cooperation. In the next chapter, I discuss some potential mechanisms through which the mere perception of moving together in synchrony could increase cooperation and I attempt to assess these mechanisms by measuring some key mediating psychological variables. 


\section{Chapter 4 \\ SYNCHRONY UNDER MINIMAL CONDITIONS}

\section{Is group synchrony sufficient to increase cooperation?}

In my first three studies I examined whether group synchrony fosters cooperation even under conditions in which other social, cultural, and emotional variables are minimised. This helped assess whether simply being in time with others is sufficient to foster cooperation, or whether cooperative effects depend on other social, cultural, or emotional variables. The positive social effects found from dyadic synchrony studies involving the simple manipulation of whether one's finger movements are in time with another suggests that relative timing is critical (Hove \& Risen, 2009; Valdesolo \& DeSteno, 2011). However, it is not clear whether the same processes that produce social effects in dyadic synchrony also apply to groups. In a dyadic interpersonal situation participants are able to give their full attention to their interaction partner. In a group setting, attention is more diffuse amongst the group members. Moreover, in a group activity the interpersonal relationships between any two members may not be as important as the overall group identity. Such collective identity may produce social effects from synchrony that would not otherwise be present in dyadic synchrony. Although Wiltermuth and Heath (2009) examined group synchrony, as discussed in the previous chapter, the use of a national anthem to synchronise made it unclear what role synchrony played in increasing cooperation.

\section{How might group synchrony increase cooperation?}

Through what mechanism might simply being in time with others be sufficient to increase cooperation? Here I examine the potential role of three possible mediating variables: perceived similarity, interdependent self-construal, and entitativity. 


\section{Perceived similarity}

One avenue through which synchrony could foster cooperation is perceived similarity. Valdesolo, Ouyang, and DeSteno (2010) found that participants who rocked chairs in synchrony rated feeling more similar in various personality attributes to their partner than those in the asynchrony condition. Valdesolo and DeSteno (2011) also found the same result with simple finger tapping, but moreover, feelings of similarity mediated feelings of compassion which in turn predicted greater helping behaviour. How might synchrony induce feelings of being similar in personality attributes? One possibility is based on the perceptionaction link. Our perception system and motor system are tightly linked, with perception of another performing an action increasing the probability that the perceiver would perform that action (Prinz, 1997). The discovery of mirror neurons which fire both when an action is conducted and when the same action is perceived supports this prediction (Di Pellegrino, Fadiga, Fogassi, Gallese, \& Rizzolatti, 1992). This perception-action link has been used to explain, in-part, our ability to imitate (Iacoboni, 2009) including the phenomenon of mimicry (see Chartrand \& van Baaren, 2009). The common coding for both self-generated and othergenerated behaviour proposed by the perception-action link raises the question of how do people distinguish their own action from that of another? Hurley (2005) in her Shared Circuits Model suggests differentiating self-generated action from other-generated action is dependent on: (1) monitoring whether there is inhibition of automatic mirroring, and (2) whether predicted feedback from an action matches actual feedback from a perceived action (see also Hurley, Clark, \& Kiverstein, 2008). If a behaviour is inhibited or does not match predicted feedback the brain classifies the behaviour as externally generated. No inhibition or the predicted and actual feedback match, indicates to the brain that the behaviour is internally generated. Hove (2008) suggests that in cases when perceived behaviour is not inhibited, for example with dyadic mimicry, the other person's behaviour may - at some level of neural processing - be attributed to the self. This could result in a blurring of the self with another. Likewise, when the timing of another's action matches the expected timing of one's own action, for example with phase-locked interactional synchrony, this could also result in selfother blurring (Hove, 2008; Smith, 2008). When both timing and form are matched (i.e., moving in the same way at the same time) this could lead to particularly pronounced sensations of another's actions feeling like they are yours.

The importance of timing for the creation of a sense of self can be seen in bodily illusions. The rubber hand illusion (Botvinick \& Cohen, 1998) occurs when a person 
perceives a rubber hand being stroked with a paintbrush at the same time as their own visually occluded hand is also being stroked. People perceive the stroking sensation as coming from the rubber hand, as if they had integrated it into their own person. A similar illusion has also been found when movement of a real arm is synchronised with movement of a fake arm (Dummer, Picot-Annand, Neal, \& Moore, 2009). Other studies have found this effect generalises to the face and even the entire body (Blanke \& Metzinger, 2009; Sforza, Bufalari, Haggard, \& Aglioti, 2010). Importantly, this illusion also leads to increased feelings of closeness to another and increased similarity of personality traits based on projection of their own personality and inner states (Mazzurega, Pavani, Paladino, \& Schubert, 2011; Paladino, Mazzurega, Pavani, \& Schubert, 2010). This suggests that moving in synchrony could lead to an expansion of the self to include another person such that a person projects their own characteristics on to another and sees similarities in another based on their own attributes (Smith, 2008). Such an effect could explain why there is an increase in perceived similarity in personality attributes after synchrony.

The next question is why would perceived similarity lead to greater cooperation? Valdesolo and DeSteno (2011) suggest that people might use similarity as a cue that someone will likely reciprocate in a cooperative interaction - although it is not clear how similarity might have become such a cue. Notably, both perceived attitudinal and physical similarity are used as cues of genetic relatedness (Bressan, Generale, \& Padova, 2009). Through the process of kin selection (Hamilton, 1964), people might have evolved a tendency to cooperate with people that are perceived as similar to them because people who are similar are more likely to have been related to them. Another possibility centres on projection: perceiving our personal attributes in another person might allow for richer representations of that person's mental processes and so could help facilitate empathy (Preston \& de Waal, 2002). Such empathy, might motivate people to help another (Van Lange, 2008). Support for an empathy-mediated mechanism comes from evidence in Valdesolo and DeSteno's (2011) study that found that compassion mediated the similarity-cooperation relationship. A third, and perhaps related mechanism, operates through trust: if I feel that another is similar to me, I might believe that the other person will be more likely to act cooperatively if I do. This amplification of trust might then increase my confidence in predicting reciprocity. 


\section{Interdependent self-construal}

Another potential variable mediating the synchrony-cooperation relationship is interdependent self-construal. Self-construal in general refers to "how individuals define and make meaning of the self" (S. Cross, Hardin, \& Gercek-Swing, 2011, p. 143). Markus and Kitayama (1991) suggested that a person's self-construal could be classified as independenta person perceives their self as unique and separate from others; or interdependent - a person perceives their self in terms of their relationships to other people. Self-construal was initially examined as more of a dispositional variable, with a particular focus on differences in selfconstrual between cultures (e.g., Markus \& Kitayama, 1991). Further studies have found that priming people to focus on others increases interdependent self-construal (Gardner et al., 1999; Stapel \& Koomen, 2005). Of particular relevance here, Ashton-James et al. (2007) found that mimicry modified people's self-construal, with participants who had been mimicked having a more interdependent self-construal. Although mimicry and synchrony are different phenomena, shared perception-action circuits have been argued to underlie both (Chartrand \& van Baaren, 2009; Hove, 2008). It is therefore possible that synchrony, too, might increase interdependent self-construal. One way that synchrony could increase interdependent self-construal is through the same self-other blurring produced through shared action-perception systems as described above. After synchronising with another, a person might perceive their self as less distinct from another. Another possibility is that the increased similarity that one feels after performing synchrony could lead one to feel as if their self is not so unique, but more connected to others. Being in synchrony could also affect people's cognition in more general ways that in turn influence their self-construal. For example, after being mimicked, people process information in a more context dependent manner (Van Baaren, Horgan, Chartrand, \& Dijkmans, 2004). In both synchrony and mimicry this shift in cognitive style could be because the similarity people observe in others' movements draws their attention to the social context. An increase in awareness of context is strongly associated with interdependent self-construal (Kühnen, Hannover, \& Schubert, 2001).

Interdependent self-construal has been found to result in increased cooperative behaviour. Utz (2004) primed interdependent self-construal by asking participants to unscramble words such as friendship or together. Those participants who unscrambled such relationship orientated words cooperated more in a social dilemma game. Ashton-James et al. (2007) found that an interdependent self-construal mediated the relationship between mimicry and cooperative behaviour. Of note, Ashton-James et al. found that participants after 
being mimicked felt more connected to other people in general, not just the person who mimicked them. Such generalised self-construal could explain the finding that mimicry results in increased cooperation towards not only the person that was mimicked or did the mimicking, but to a stranger who they had never seen before or faceless charity organisations (Ashton-James et al., 2007; Stel et al., 2008; Van Baaren, Holland, et al., 2004).

How could a shift in interdependent self-construal lead to greater cooperation towards other people? As well as increased similarity leading to greater self-construal, the reverse could also be true: self-construal might mediate the relationship between synchrony and similarity. An independent self-construal defines the self as unique and therefore different from others, whereas an interdependent self-construal sees the self as connected to others with shared features. Indeed, people who have interdependent self-construal personalities see more similarities with others than those with more independent self-construal (Kühnen, 1999, cited in Kühnen \& Hannover, 2000). Likewise, subtle priming of interdependent selfconstrual also results in participants seeing more similarities with others (Kühnen \& Hannover, 2000). Synchrony might then increase interdependent self-construal, which would increase perceived similarity, which in turn increases cooperation (through the mechanism described on page 35).

Interdependent self-construal might also increase cooperation through empathy. A number of studies on chronic or manipulated self-construal have found that a more interdependent self-construal is connected with thinking about other people more in terms of their knowledge, views, wishes and desires (Gore \& Cross, 2006; Haberstroh, Oyserman, Schwarz, Kühnen, \& Ji, 2002; Ybarra \& Trafimow, 1998). For example, Utz (2004) found that a desire to maximise another's gain in a social dilemma game mediated the relationship between self-construal and cooperation. Thinking more about others could lead to a greater tendency to empathise with them and therefore motivate one to help or cooperate. Thinking about other people may also increase awareness of social norms. Certainly, previous research has found that interdependent self-construal has been linked with a greater motivation to conform and follow social norms (Haberstroh et al., 2002; Wiekens \& Stapel, 2008). If people are placed in a situation in which helping or cooperating is the norm or the socially desirable behaviour, those with interdependent self-construal may be more likely to cooperate. 


\section{Entitativity}

A mediating variable that may be of particular importance with group synchrony is entitativity. Entitativity is the degree to which a collection of people are perceived as a group. The term was coined by Campbell (1958), who argued that the same cognitive processes through which people perceive objects in the world as entities can also be applied to the perception of groups of humans. For example, we group together elements that are similar, elements that are close together, elements that have good spatial organisation, and elements that move together in the same direction. A number of these processes could be directly relevant to group synchrony, in particular, elements that move in the same direction. Group synchrony, then, might engage heightened feelings of entitativity through human's evolved perceptual system grouping together people, giving performers the feeling that they are not separate entities but a collective. In support of synchrony increasing entitativity, Lakens (2010) found that the perception of dyadic synchrony appears to increase perceived entitativity with real or schematic people. Likewise, Wiltermuth and Heath found in their third experiment that synchrony increased feelings of being on the same team. Moreover, Wiltermuth and Heath found that feeling as a team partially mediated the synchronycooperation effect that they found.

Entitativity could increase cooperation through influencing participants' group identity. The idea that certain factors can trigger group identity links with self-categorisation theory (J. C. Turner, Hogg, Oakes, Reicher, \& Wetherell, 1987). Self-categorisation theory posits that people can perceive themselves as having personal identities and various social identities. As social identities are made more salient, depersonalisation of the self occurs, which involves: "a cognitive redefinition of the self - from unique attributes and individual differences to shared social category memberships and associated stereotypes" (Turner, 1984, p528). Self-categorisation theory was based predominantly on categorisation with existing groups for which stereotypes already exist. However, similar processes could also exist for new groups, as research on minimal groups suggest (e.g., Billig \& Tajfel, 1973).

If group synchrony does increase group identity, this would link in with a well-known finding in social psychology that even minimal cues of belonging to a group result in increased cooperation with-in that group (Billig \& Tajfel, 1973; Kramer \& Brewer, 1984; Yamagishi \& Mifune, 2009). A redefinition of the self in terms of a group identity could result in a reduced feeling of an $I$ that could be cheated during a cooperative interaction, and an increased feeling of a collective we that share the same fate (Dawes, Van de Kragt, \& 
Orbell, 1988; De Cremer, van Knippenberg, van Dijk, \& van Leeuwen, 2008). The creation of a group identity could also result in participants thinking more about the wishes and desires of the other group members and in greater perceived similarity of personality traits (Coats, Smith, Claypool, \& Banner, 2000; Otten \& Epstude, 2006; Smith \& Henry, 1996).

Similarity, interdependent self-construal, and entitativity could therefore all be important mediating variables in explaining why synchrony increases cooperation. However, because these constructs are all closely related it may be difficult to elucidate the exact path from which one may cause another. One differentiation that might be possible is whether performing in group synchrony results in people feeling closer to other people in general (generalised interdependent self-construal) or produces a group identity through entitativity. A shift in generalised interdependent self-construal could be measured by more generalised cooperation, for example an increase in prosocial behaviour towards another who is not part of the immediate performance group. A shift in generalised cooperation has so far been investigated only for dyadic mimicry (e.g., Stel et al., 2008; van Baaren et al., 2004). Whether synchrony increases cooperation to others outside of those performing synchrony has yet to be established. If the synchrony-cooperation effect is mediated by entitativity, this could suggest that increased cooperation would be limited to the performance group. A large number of studies using the minimal group paradigm have found group-specific discriminations from subtle indications of group membership (see reviews by Hewstone, Rubin, \& Willis, 2002; Mullen, Brown, \& Smith, 1992). An entitativity mediated mechanism may be especially of relevance with group synchrony as commonly seen in music and dance.

\section{Experiment 1}

\section{Overview}

My first experiment had three main aims: (1) to determine if the group synchronycooperation effect could be reproduced under minimal conditions; (2) to assess whether an increase in cooperation is restricted to the performance group or generalised to nonperformers; and (3) to examine whether synchrony also affects some of the proposed mediators. Following Wiltermuth and Heath's procedure, a synchrony condition was compared to two control conditions: one in which participants moved at different speeds (asynchrony) and one where participants were not required to move at all (they sat passively 
watching a video). The synchrony and asynchrony conditions were similar in all respects except the relative timing of participants' movements. The passive no-movement condition was used as a baseline to assess the cooperative tendencies of a group of people who are in the same room doing the same activity (watching a video of people moving) but not moving or interacting in anyway.

Cooperation was measured with a helping scenario that involved a confederate asking participants if they were willing to take part in an unrewarded study. To examine whether increased cooperation is restricted to the performance group or more generalised, I used two conditions: one where the confederate took part in the activity (performance condition), and another where the confederate came in afterwards (non-performance condition). The experiment was therefore a factorial design with two independent variables (IV): movement (the synchrony manipulation), and membership (whether or not the confederate is part of the performance group). I then compared two hypotheses: (1a) that the synchrony condition will increase cooperation towards both a performance and non-performance member (generalised cooperation); (1b) that the synchrony condition will only increase cooperation towards a performance member (group-specific cooperation). Hypothesis 1a predicted that there should be a main effect of the synchrony manipulation on helping, but no interaction between the movement IV and the membership IV. Participants in the synchrony condition should be more willing to help the confederate irrespective of whether the confederate participated in the activity or not. Participants in the synchrony condition should be willing to donate significantly more time to help the confederate than participants in both the asynchrony and passive conditions. Hypothesis $1 \mathrm{~b}$ predicted that there should be a significant interaction between the movement IV and the membership IV. It predicted that the level of cooperation in the synchrony condition would only be significantly greater than the asynchrony and passive conditions when the confederate is part of the performance group. There will be no significant differences across conditions in the non-performance condition.

Perceived similarity and interdependent self-construal were measured through selfreport questionnaires. Interdependent self-construal was targeted at a number of different groups including other people in general and the performance group. ${ }^{7}$ Hypothesis 1a predicted that a higher level of perceived similarity and interpersonal self-construal would be seen in the synchrony condition compared to the other two conditions for both the performance and non-performance conditions. Hypothesis 1b, predicted that a higher level of

\footnotetext{
${ }^{7}$ Entitativity was not directly measured in experiment 1 , but indirectly measured through interdependent selfconstrual directed towards the performance group.
} 
perceived similarity would only be seen in the performance condition along with a higher level of interdependent self-construal targeted only at the performance group.

\section{Method}

\section{Participants}

Eighty-two undergraduate psychology students (61 female; mean age $=20.36$ years, range: 18-70 years) participated in the study for course credits in groups of three or four. Participants signed up for the experiment through a website and chose from a limited range of experimental sessions. Groups were randomly assigned to a particular condition. The distribution of females in each cell of the factorial design was reasonably even varying from 9 to $12 .^{8}$

\section{Procedure}

A $3 \times 2$ between subject design was used, with three conditions of the movement IV (synchrony, asynchrony, and passive) and two conditions of the membership IV (performance and non-performance). The dependent measure of cooperation was tested directly after the synchrony manipulation. After this measure, participants filled out a questionnaire (post-activity questionnaire) which included demographic questions, self-report scales to test control variables, and self-report scales to test potential mediating variables.

\section{Synchrony manipulation}

For the synchrony and asynchrony conditions, participants were asked to do three different simple movements each lasting two minutes: (1) move their left arm up from a resting position at the side of the body and then down, then their right arm up and down; (2) sway their upper body from an upright position to the left and up, then to the right and up; and (3) move their left leg up from a standing position and down, then their right leg up and down. The use of different movements was to prevent muscle fatigue and boredom. Participants stood in a row to try and reduce emotional contagion from observing each other's facial expressions. However, they could still observe each other's movements through their

\footnotetext{
${ }^{8}$ A log-linear analysis with movement, membership and sex found no significant interactions, only a main effect of sex (there were more females than males in the sample).
} 
peripheral vision. I used simple body movements (adapted from Naruse \& Hirai, 2000) rather than the finger tapping paradigm used by Hove and Risen (2009) and Valdesolo and DeSteno (2011) as body movements should be easier to observe in a group. Body movements also more closely approximate the synchrony observed in group dance. It was anticipated that the movements would need minimal strength and flexibility so should be able to be performed by most people. The activity took a total of six minutes.

Participants were asked to move in time with a metronome beat played through headphones. The metronome beats were written on ProTools version 7.1. The beat was in 4/4 time signature (four beats per repetitive unit) with the first beat accented by using a higher pitched and louder percussion tone than the following three beats. Metronome beats were converted to $\mathrm{mp} 3$ format and copied on to a portable digital audio player. In the synchrony condition, group members heard the same metronome beat being played through their headphones (tempo at 65 beats per minute [bpm]). This was accomplished by using a fourway headphone splitter, allowing all four headphones to be connected to the same digital audio player. Participants were also instructed to always start on their left with the first accented beat so as to create perfect synchrony. In the asynchrony condition, a slightly different tempo was played through the headphones from different digital audio players resulting in the participants moving out of synchrony with each other. These tempos were at $60 \mathrm{bpm}, 65 \mathrm{bpm}, 70 \mathrm{bpm}$, and 75bpm (if the group had three participants the $75 \mathrm{bpm}$ tempo was not used). I conducted a small pilot study testing a variety of different tempos and found that this range of tempos was the easiest and most comfortable for the participants to perform the movements to. These particular tempos also produced complex ratios with each other which would result in minimal incidental momentary synchrony - i.e., when 2 or more participants would occasionally come into phase with each other. For example, if I used a tempo of $30 \mathrm{bpm}$ and $60 \mathrm{bpm}$ then every second movement of the participant moving at $60 \mathrm{bpm}$ would coincide with the participant moving at $30 \mathrm{bpm}$. The tempos were also different enough that movements would quickly diverge if any incidental momentary synchrony did occur. Graphical modelling in Excel software of the resulting sine waves produced by these frequencies confirmed that they would produce minimal synchrony.

The experimenter gave a brief demonstration with instructions on how to synchronise the movements with the beat by playing the metronome rhythm over computer speakers. Four tambourine beats were played just before the metronome beat began to prepare participants for the tempo so as to help them synchronise to the beat. On the first beat the left side (arm, body or leg) was moved. On the second beat it came back to rest. The right side was moved 
on the third beat. On the fourth beat it came back to rest again. Participants then practised each of the movements twice to make sure they understood the instructions. In the synchrony condition, participants were informed that they would hear the same beat as the other participants and in the asynchrony condition that they would hear different beats. Participants were instructed to try and keep in time with the beat being played through their headphones. The order of movements was always arms, body and legs. This was written on a board in front of the participants to remind them of the order. During the activity the beat was temporarily suspended at two minute intervals, at which time participants were instructed to change to a new movement.

In the passive condition a video was created of four people doing the same movement activity in synchrony with an audible metronome click in the background. Participants sat in a row on four chairs whilst watching this video for approximately six minutes.

\section{Cooperation measure}

Cooperation was assessed with a helping measure adapted from Ashton-James et al. (2007). A female confederate, who was unaware of the experimental hypothesis, was used to recruit volunteers for a subsequent unrewarded study. In the performance condition, the confederate took part in the group activity. She was introduced before the activity under the guise that a certain number of participants were needed for the group activity and as not enough participants had signed up she had volunteered to fill in. In the non-performance condition, the confederate was introduced after the movement manipulation. The experimenter informed participants (who were seated in chairs): "next you will need to fill in a short questionnaire, but while I am organising these questionnaires my colleague who has been helping me with my study will talk to you for a few moments about a study that she is involved in." The confederate then handed participants a sheet outlining the unrewarded study. The confederate read from the sheet to make sure participants in all conditions received the same information. Participants were asked if they were willing to complete a voluntary and anonymous on-line survey. Because participants may have had lectures or other commitments directly after the experiment it was decided to send participants a link for an on-line survey rather than ask volunteers to fill out the survey then and there. Participants could choose to complete either a 20 minute survey, a 40 minute survey, or could indicate that they would not like to take part in either survey. The two lengths of survey were offered to help assess the magnitude of any prosocial response i.e., choosing the longer survey would indicate that they were willing to donate more time. To emphasise that the survey was 
unrewarded, participants were told that they would not receive course credits nor would they be paid, but any help they were willing to give "would be very much appreciated and invaluable to the project". Participants anonymously indicated on the form which version (if any) they would be willing to complete, along with their email address. Participants then placed the form into a sealed box. After the experiment, the recruitment sheets for the unrewarded study were marked as to the condition the participant was in. Within 24 hours of a group completing their activity, participants who were willing to partake in the unrewarded survey were emailed a link to an internet based survey. The email stated that the questionnaire should take no more than 20 minutes or 40 minutes - depending on which survey they had volunteered to do; however, both surveys were exactly the same.

\section{Post-activity questionnaire}

After the cooperation measure, participants completed the post-activity questionnaire. This questionnaire involved scales to measure perceived similarity and interdependent selfconstrual. Perceived similarity was measured with the attitude homophily subscale (Cronbach alpha $=.86$ ) from the homophily scale (McCroskey, McCroskey, \& Richmond, 2006). The scales were adapted slightly so that the target was the performance group rather than a singular person. Participants were asked to rate on a 7-point Likert scale from 1 (strongly disagree) to 7 (strongly agree) how strongly they agreed or disagreed with 15 items (7 reverse coded) about the people they had just done the activity with. For example: "these people have thoughts and ideas that are similar to mine".

Self-construal was measured in two different ways: with the Six-fold self-construal scale (Harb \& Smith, 2008) and the Inclusion of the Other in the Self scale (IOS; Aron, Aron, \& Smollan, 1992). The Six-fold Self-construal scale integrates self-construal as per Markus and Kitayama (1991), self-categorisation theory (Turner et al., 1987), and Brewer and Gardener's (1996) tripartite self-representational model which includes relational, collective and personal self-definitions. It measures self-construal on four key levels: personal, relational, collective self, and humanity. The humanity self is when "the self is defined by its belonging to the human species...this overarching category transcends the norms and properties associated with social interactions (both relational and collective) and rests on a universal representation" (Harb \& Smith, 2008, p. 183). The scale involves five questions asking about participants' relationships with six groups representing these four different levels: their own self (personal), family (relational), friends (relational), students at their university (collective), social grouping (collective), and humanity in general (humanity). I 
modified the social grouping subscale to be "the people I have just done the activity with" to directly measure participants' self-construal towards their activity group. Because participants have a limited history with the performance group I removed the questions: "I control my behavior to accommodate the wishes (interests) of " and "I am affected by events that concern (relate) to " which would not make sense in this context. The remaining three questions were: "I think of myself as connected (linked) to "; "I am aware of the needs, desires and goals of "; "I feel I have a strong relationship with ". Participants were asked to respond on a 7-point Likert scale from 1 (to a very small extent) to 7 (to a very large extent). Despite the reduction in the number of questions for each subscales from five to three, the reliability for each of the subscales was still good (Cronbach alphas: family $=.88$; friends $=.84 ;$ students $=.87$; performance group $=.75$; humanity $=.87$; myself $=.78)$. A principle components analysis with varimax rotation still found six factors matching the six subscales (lowest factor loading $=.69$, factors explained $77.61 \%$ of the variance).

The IOS was developed as a tool to measure closeness in interpersonal dyadic relationships. As feelings of connectedness are a key characteristic of an interdependent selfconstrual (Markus \& Kitayama, 1991) the scale has also been used as a measure of selfconstrual (e.g., Ashton-James et al. 2007) and was used to validate the six-fold self-construal scale (Harb \& Smith, 2008). The one item scale asked participants to rate how close they feel to a targeted other. It involved seven pictures of two circles - one labeled self, the other circle labeled other - with various degrees of overlap (see Figure 1 on page 46). I used two different versions of the IOS. One version asked participants to select the picture that best describes how close they currently feel to all the people they just did the activity with. This version was used to measure interdependent self-construal specific to the performance group. The second version asked participants to rate how close they feel to other people in general. This second version was used to measure generalised interdependent self-construal. The sixfold self-construal measure targeted at the performance group was significantly correlated with the IOS targeted at the performance group $(r=.33, p<.01)$. Likewise, the six-fold selfconstrual measure targeted towards humanity was significantly correlated with the IOS targeted at other people in general $(r=.31, p=.01)$. 

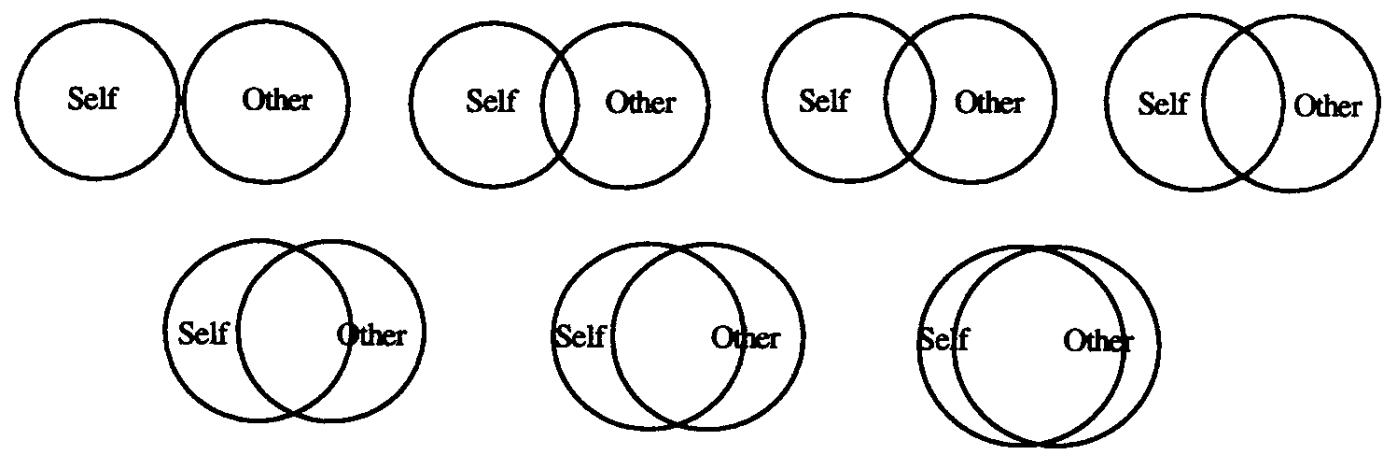

Figure 1. The Inclusion of Other in the Self scale (IOS). Note. From Aron, Aron, and Smollan (1992).

To check that my synchrony manipulation was minimal and did not affect participants emotions or arousal in any way, mood was measured using the University of Wales Institute of Science and Technology (UWIST) Mood Adjective Checklist (UMACL; Matthews, Jones, \& Chamberlain, 1990). This scale involved participants rating on a 4-point Likert scale how applicable 24 adjectives were to their present mood (1: definitely not applicable; 4: definitely applicable). The scale included three subscales: hedonic tone (Cronbach alpha $=.81$ ), tense arousal (Cronbach alpha $=.76)$, and energetic arousal (Cronbach alpha $=.73)$. Each subscale had 8 items with 4 of the items reverse coded.

Because it was possible that some participants knew each other before the experiment, participants were also asked to rate how well they knew each of the other participants before the study on a 5-point scale with the following headers: 1 (I had never seen him/her before), 2 (I had seen him/her before), 3 (I had talked briefly with him/her before), 4 (I had spent time with him/her before), 5 (I know him/her very well). Because there were three or four participants in each group, this resulted in two or three ratings for each participant. To create a single summary statistic for a participant's pre-experimental bonding, both the highest rating a participant made across the participants in their group and the mean rating was used.

Two open-ended questions were also included, one asking participants to write any general comments they would like to make about the group activity they just did and one asking for comments about the survey or the study in general. 


\section{Results}

\section{Pre-experiment bonding}

Across all groups, $67 \%$ of participants had never seen any of the other participants in their group before i.e., they were total strangers. A 3 x 2 Analysis of Variance (ANOVA) using either the mean rating for each participant or the highest rating for each participant as the dependent variable with movement and membership as the independent variables found no significant main effects or interactions in how well participants knew each other beforehand $\left(F_{\max }=1.67, p=.20\right)$. This indicates that pre-experimental bonding should not be a significant factor in any differences between the experimental conditions.

\section{Cooperation measure}

The unrewarded study scenario appeared credible: $81 \%$ of participants volunteered for one of the two versions and only one participant expressed some suspicion about the volunteering measure in the post-activity questionnaire (this participant was removed from further analysis).

To compare hypothesis $1 \mathrm{a}$ and $1 \mathrm{~b}$, a 3 x 2 ANOVA was conducted with movement and membership as independent variables and cooperation (the amount of time willing to donate) as the dependent variable. The only significant effect was a main effect for movement: $F(2,75)=3.41, p=.04, \eta_{p}{ }^{2}=.08{ }^{9}{ }^{9}$ The Movement X Membership interaction was not significant: $F(2,75)=.68, p=.51, \eta_{p}{ }^{2}=.02$; nor was the main effect of membership: $F(1,75)=1.65, p=.20, \eta_{p}{ }^{2}=.02$. Planned comparisons between the synchrony $(M=28.57$, $S D=13.80)$ and asynchrony conditions $(M=23.70, S D=13.63)$ was not significant, $t(53)=$ $1.32, p=.19, d=0.36$, but the synchrony condition was significantly higher than the passive condition $(M=18.46, S D=14.88), t(52)=2.59, p=.01, d=0.72$. There was also no significant difference between the asynchrony and no-movement condition, $t(51)=1.34, p=$ $.19, d=0.38 .{ }^{10}$ Comparison of the overall means across movement conditions (see Figure 2 on page 48) shows that the greatest cooperation occurred after synchronous movement, the lowest cooperation occurring after the passive condition, with asynchronous movement in

\footnotetext{
${ }^{9}$ An alpha level of .05 is used for all analyses.

${ }^{10}$ I did not have an a priori prediction for a difference between the asynchrony and no-movement condition, but even with a more conservative correction for multiple comparisons that difference would, of course, still be nonsignificant.
} 
between. Polynomial contrast analysis found that a linear trend significantly fitted the data, $F(1,78)=6.93, p=.01$.

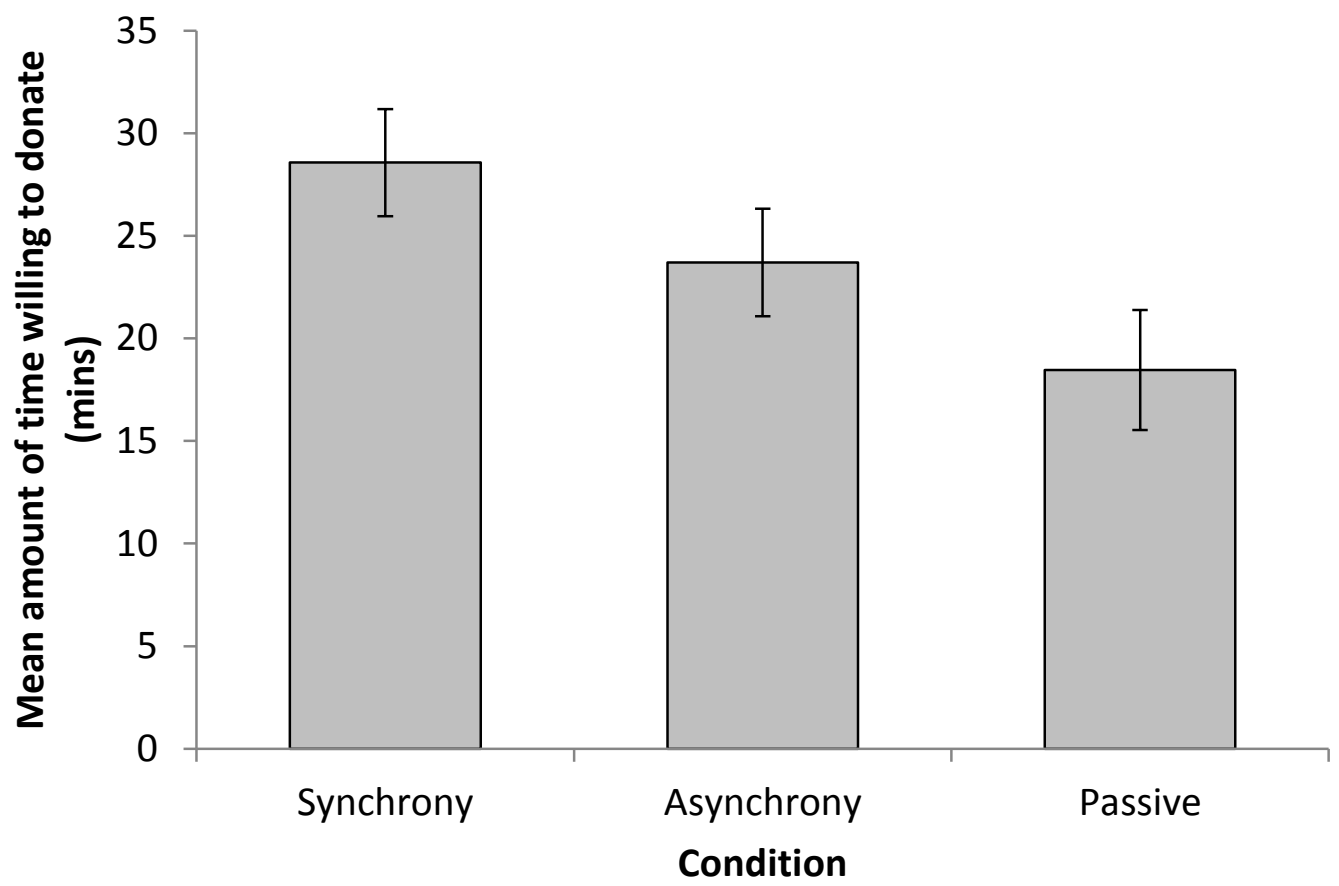

Figure 2. Mean amount of time willing to donate (minutes) for each of the movement conditions. Error bars are +/- SE.

Across conditions, $52 \%$ of participants who said they were willing to complete the survey actually went on-line and completed it. This shows that willingness to complete the questionnaire did respond to actual behavior for the majority of participants. To examine if there was any effect of movement or membership on the proportion of volunteers who completed the survey a three-way hierarchical log-linear analysis with backward elimination was conducted with movement (3 levels), membership (2 levels), and completion (2 levels: did complete, did not complete). The analysis revealed no main effects or interactions.

\section{Post-activity questionnaire}

Looking to the questionnaire measures, $3 \times 2$ ANOVAs with movement and membership as the independent variables were performed for attitude homophily (perceived similarity), IOS directed at the group, and IOS directed at other people in general. No significant main effects or interactions were found for any of these measures $\left(F_{\max }=2.05, p=\right.$ .16). To assess self-construal as measured with the six-fold self-construal scale a multivariate 
ANOVA with the six subscales as the dependent variables and condition and group membership as the independent variables was performed. ${ }^{11}$ Using Pillai's Trace, no main effects or interactions were found for condition or group membership $\left(F_{\max }=0.68, p=.77\right)$.

One-way ANOVAs ${ }^{12}$ with movement as the independent variable were performed for each of the three mood subscales. There were no significant differences with tense arousal, $F(2,75)=1.03, p=.36, \eta_{p}{ }^{2}=.03$, but there was a marginally significant main effect of condition for hedonic tone, $F(2,75)=2.80, p=.07, \eta_{p}{ }^{2}=.07$, and a significant main effect of condition for energetic arousal, $F(2,75)=3.50, p=.03, \eta_{p}{ }^{2}=.09$. For hedonic tone, Gabriel post-hoc tests ${ }^{13}$ revealed no significant differences between any of the conditions (synchrony vs. asynchrony, $p=1.00$; synchrony vs. passive, $p=.14$, asynchrony vs. passive, $p=.12$ ). For energetic arousal, Gabriel post-hoc tests found that the synchrony $(M=2.59, S D=0.48)$ and asynchrony conditions $(M=2.61, S D=0.53)$ were not significantly different $(p=1.00)$. The difference between the synchrony and passive conditions $(M=2.29, S D=0.45)$ and the asynchrony and passive conditions were marginally non-significant $(p=.08, d=0.64$, and $p$ $=.06, d=0.65$ respectively). Although the pairwise post-hoc comparisons for both hedonic tone and energetic arousal were not significant, the largest differences were found between the passive condition and the synchrony or asynchrony condition. It was these differences with the passive condition that produced the significant main effects in the ANOVAs seen with hedonic tone and energetic arousal.

\section{Discussion}

My first experiment provided data consistent with the hypothesis that moving in synchrony results in greater cooperation, even under minimal conditions. A significant main effect of movement was found, with the synchrony condition producing the highest level of helping, with a significantly greater degree of cooperation than the passive condition. The significant linear trend across the three groups is certainly suggestive of group synchrony increasing cooperation compared to asynchrony, and the effect size between the synchrony and asynchrony conditions (Cohen's $d=0.36$ ) is comparable to the difference between the

\footnotetext{
${ }^{11}$ Multivariate analysis was preferred to repeated measures as there was a large violation of sphericity $(\varepsilon>.7)$ and I had a reasonably large sample size (Field, 2009).

12 The membership variable was not included in the ANOVAs on mood as there was no reason to expect whether the confederate took part in the activity or not should have any effect on mood. My interest was primarily whether the synchrony manipulation influence mood.

${ }^{13}$ The Gabriel post-hoc test was chosen as this test has the greatest power when sample sizes are slightly different and population variance should be similar (Field, 2009).
} 
synchrony and asynchrony condition that Wiltermuth and Heath found in their third study (Cohen's $d=0.42$ ).

The significant linear effect across the three conditions in cooperative behaviour also suggests that asynchronous movement results in slightly higher cooperation compared to passive no-movement, but being in-synchrony has an even more pronounced effect. One possible reason that asynchronous movement might increase cooperation compared to the passive condition is that movement in general increases cooperation compared to passive sitting. Such an effect could be mediated by mood. For example, exercise has been linked to an increase in endorphins and a good mood (Thoren, Floras, Hoffmann, \& Seals, 1990) and a good mood in turn has been linked to greater prosocial behaviours (see Batson, 1998). The finding that the mean levels of energetic arousal in the synchrony and asynchrony conditions were higher than in the no-movement condition (although marginally non-significant) lends support to this idea. ${ }^{14}$ Asynchronous movement might also increase cooperation through a similar mechanism through which mimicry increases cooperation. Although the movements of participants in the asynchrony condition were not matched in time, the specific behaviours they performed were matched in form. Data from mimicry studies indicate that moving in the same way even out of time increases prosocial behaviour (e.g., Van Baaren et al. 2004).

What do the data suggest about the generalisability of the effect? The finding of a main effect of synchrony but no significant main effect of membership nor a Movement $\mathrm{x}$ Membership interaction lends greater support to hypothesis 1a: that moving in synchrony results in a generalised prosocial effect. Examining the individual cell means, for none of the movement conditions was cooperation greater in the performance condition than in the nonperformance condition. The finding of a generalised prosocial effect could suggest that the cooperative effects from synchrony are mediated by a shift towards a more generalised interdependent self-construal, rather than synchrony increasing a group specific interdependent self-construal. However, the lack of significance across conditions with any of the potential process variables I measured provides no support for synchrony affecting selfconstrual or perceived similarity.

A potential confound in the experimental design comes from the use of a confederate in the performance condition. Although the confederate was naïve to the hypothesis, she may have unintentionally adapted her behaviour dependent on the condition of the synchrony

\footnotetext{
${ }^{14}$ Because the volunteering measure was completely anonymous participants' responses in the self-report measure could not be linked to their behaviour. Therefore any mediational analyses to investigate the role energetic mood may have played could not be conducted.
} 
manipulation. For example, performing the passive no-movement condition multiple times may have induced boredom in the confederate resulting in a less enthusiastic recruitment of participants, so producing a lower level of helping in the passive condition. In the next experiment I used another measure of in-group cooperation which does not involve a confederate so as to by-pass this issue.

The lack of a significant difference between the synchrony and asynchrony condition in both behavior and self-report variables are in contrast to Wiltermuth and Heath's (2009) findings. One possibility for why no significant differences were found was due to the minimal nature of the synchrony manipulation. Other variables such as shared emotion and cultural group identifiers might have helped accentuate the small effect in cooperation that I found. Another possibility is that the modality of synchrony is important. In Wiltermuth and Heath's second and third study all of their active conditions (i.e., synchronous singing, synchronous singing and moving, asynchronous singing and moving) involved vocalising. Synchronised vocalising may have a stronger effect than synchronised movement. There are a few reasons to suspect why this could be the case. Firstly, humans appear better at entraining to auditory rhythms than visual rhythms (Repp \& Penel, 2004). This could be because people more commonly entrain to another's auditory rhythms (both in terms of language, e.g., entraining to speech rhythms, and musical productions) and therefore more readily interpret synchronous vocalising behaviour as indicative of affiliation (see page 13). Secondly, people perceive synchronised movement through the visual channel which is much more focused than audition. It is not possible for humans to take in all of the visual information that is around them - people are restricted by the direction their head is facing. Even within a visual scene the foveal region that we can focus on is quite small. Audition, on the other hand, is the sum of all the auditory information that is occurring around us irrespective of which direction our head is facing. In terms of synchrony, this means that to assess or create synchronous movement people need to be facing and focusing on the whole group. This may not be possible for someone participating in a dance. In my first experiment, participants may have been facing forward ignoring the other participants or focusing on just one of the other participants. That is, they may not have been fully attentive to the synchrony happening around them. With vocalising it is difficult to ignore other people - participants will always be aware if they are in synchrony or not. This difficulty in completely ignoring other participants who are vocalising could also make the asynchronous condition more difficult. The discordance produced by asynchronous rhythmic chanting could produce frustration and annoyance in participants leading to reduced cooperative behaviour. Many 
voices also build a greater sound, so synchronised vocalising may produce a greater sensation of creating something together than synchronised movement. Therefore, in my next study I investigated the effects of synchrony in a vocalisation task.

\section{Experiment 2}

\section{Overview}

In experiment 2, I created synchronised vocalising instead of synchronised movement, with the prediction that this would produce a larger synchrony-cooperation effect. As with experiment 1, synchronised vocalising was compared against two control conditions (vocalising manipulation): asynchronous vocalising and passive listening of rhythmic vocal chanting. Cooperation towards both fellow performance members as well as towards a nonperformance member was measured. The same volunteering measure used in experiment 1 was used to measure cooperation towards non-performance members (volunteering measure). Because of the potential confound in having the confederate take part in the performance, another naturalistic cooperation measure was trialled. This involved participants being asked to complete a jigsaw puzzle together (puzzle measure). The measure of cooperation was the number of times a participant spontaneously helped another participant in trying to complete the puzzle. All participants took part in both measures.

If vocalised synchrony results in an increase in generalised cooperation (hypothesis 1a) there should be a significant main effect of vocalising with both the volunteering measure and the puzzle measure. Specifically, it was predicted that participants in the synchrony condition will help significantly more than participants in the asynchrony and passive conditions. Based on the results of experiment 1, a significant linear effect was also predicted, with the asynchrony condition producing a significantly higher level of cooperation than the passive condition.

If vocalised synchrony results in an increase in cooperation only directed towards a fellow performance member (hypothesis $1 b$ ), there will only be a main effect of vocalising with the puzzle measure. It was predicted that the synchrony condition would produce significantly more spontaneous helping in the puzzle measure than the other conditions. There should be no main effect with the volunteering measure. Again, a linear effect would be predicted with the asynchrony condition producing a significantly higher level of cooperation than the passive condition. 
Perceived similarity, interpersonal self-construal directed towards the performance group, interpersonal self-construal directed toward other people more generally, and entitativity were measured through self-report questionnaires. Perceived similarity was predicted to be greater in the synchrony condition than the other two conditions. Hypothesis 1a predicted a higher level of generalised interpersonal self-construal would be seen in the synchrony condition compared to the other two conditions. Hypothesis $1 \mathrm{~b}$, predicted that a higher level of self-construal directed at the performance group and entitativity will be seen in the synchrony condition compared to the other two conditions.

\section{Method}

\section{Participants}

Ninety-one undergraduate psychology students (62 female; mean age $=20.43$ years, range: 17-45 years) participated in the study for course credits. Participants were recruited through the same method as described in experiment 1 . The distribution of females was evenly spread across conditions. ${ }^{15}$

\section{Procedure}

A between subject design was used, with 3 conditions of vocalising (synchrony, asynchrony, and passive). Participant groups were randomly assigned to one of the three conditions. Two dependent measures for cooperation were used - the amount of time participants were willing to freely offer to an unrewarded charitable task (volunteering measure) and the number of instances of spontaneous cooperation on a collaborative jig-saw puzzle task (puzzle measure). The volunteering measure was tested directly after the synchrony manipulation. Participants then completed the post-activity questionnaire with demographic questions and self-report scales measuring potential mediating variables and control variables. The puzzle measure was conducted last after the questionnaire.

\footnotetext{
${ }^{15}$ A chi-square analysis found no difference in the proportion of females across groups, $\chi^{2}(2, \mathrm{~N}=89)=0.30, p=$ .86 .
} 


\section{Synchrony manipulation}

For the synchrony and asynchrony conditions, participants in groups of three or four were asked to read out loud a list of 59 English words (see Appendix 1 on page 139) while seated in chairs. Words were selected from the Affective Norms for English Words database (Bradley \& Lang, 1999) - a database of 1034 words with mean ratings of emotional valence from 1(negative) to 9 (positive) and emotional arousal from 1 (low) to 9 (high) for male raters and female raters. To make the stimulus as minimal as possible, emotionally neutral words were chosen. Emotionally neutral was classified as words with a mean emotional valence rating between 4 and 6 and a mean emotional arousal rating less than 5 (for both males and female ratings). Only one syllable words were selected from these so as to make synchronising pronunciation easier. The word 'board', sounding like 'bored' (with a combined emotional valence of 2.95), was also removed as it was thought it might increase thoughts of boredom in what was a potentially monotonous task. Participants in the synchrony condition were given the same sheet of words listed in a random order. In the asynchrony condition participants were given different sheets with the words in different random orders. Each participant was given a pair of headphones through which a metronome beat was played from a digital audio player. Participants were instructed to read the words in time with the beat and given a brief demonstration on how to do so by the experimenter.

In the synchrony condition, all group members heard the same metronome beat being played from a digital audio player through their headphones with the use of a 4-way headphone splitter. This meant that all participants heard the same beat at the same time and so were able to speak the same words at the same time. Similar to experiment 1 , the metronome beat was in $4 / 4$ time signature with the first beat accented. The tempo of the metronome beat was at $45 \mathrm{bpm}$. Four tambourine beats were played initially to give participants an indication of the speed of the beat before the metronome started. This metronome beat lasted 6 minutes. Participants were asked to try and read in-time with the beat. The headphones sat lightly on participants' ears so participants were able to hear each other vocalise.

In the asynchrony condition, a slightly different tempo was played through the headphones from different digital audio players resulting in the participants speaking out of synchrony with each other. These were at a tempo of $40 \mathrm{bpm}, 45 \mathrm{bpm}, 50 \mathrm{bpm}$, and $55 \mathrm{bpm}$. A small pilot study testing a variety of different tempos found that this range of tempos to be the most comfortable to read at. Participants were instructed to try and keep in time with the 
beat and not the other participants. As with experiment 1, these tempos produced complex ratios meaning any incidental momentary synchrony was limited.

In the passive condition participants listened to a recording of one person reading out loud the list of words at 45bpm. This lasted 6 minutes.

\section{Volunteering measure - generalised cooperation}

The same prosocial behavioural measure as used in experiment 1 was also used in experiment 2 but with a slight variation: to help increase the variance, participants were given four survey lengths that they could choose from depending on how much time they were willing to give - 10 minutes, 20 minutes, 30 minutes, or 40 minutes. Participants were informed that "the longer surveys will provide vital added detail that will be invaluable to the study". Participants could also choose to not take part in any survey. A confederate was used to recruit participants for the unrewarded study. The confederate was always a nonperformance member. As the confederate had to enter the laboratory immediately after the chanting activity half way through the experiment, the pretence was created that the experimenter had to leave the room to calculate a variable from the first (chanting) activity, and that while participants were waiting "a colleague of mine who has been helping me with this study will speak to you for a moment about a study he's doing”. Within 24 hours after participating in the experiment, participants who expressed a willingness to partake in the unrewarded survey were emailed a link to an internet based survey.

\section{Puzzle measure - cooperation directed towards in-group members}

For the puzzle measure, participants in their groups were given two minutes to put as many pieces of a jigsaw puzzle as they could correctly in its place. The jigsaw puzzle was a 60 piece puzzle with cartoon characters from the Ben Ten Alien Force television series (see Figure 3 on page 56). Participants were videotaped during the activity. Two independent coders, naïve to the hypothesis, counted from the videos the number of instances of spontaneous helping during the two minute puzzle activity (intra-class correlation $=.70$ ). The coding criterion was: "The number of times a participant initiated helping another participant to place a piece. This may be actually touching the piece(s), going to help another move a piece but not actually touching (e.g., withdraws because they do not need help), pointing to a position, or saying directions such as 'I think that piece goes in the corner'". Coders discussed any disagreements until consensus was reached. One group (from the asynchrony condition) was not video-taped due to a camera malfunction. 


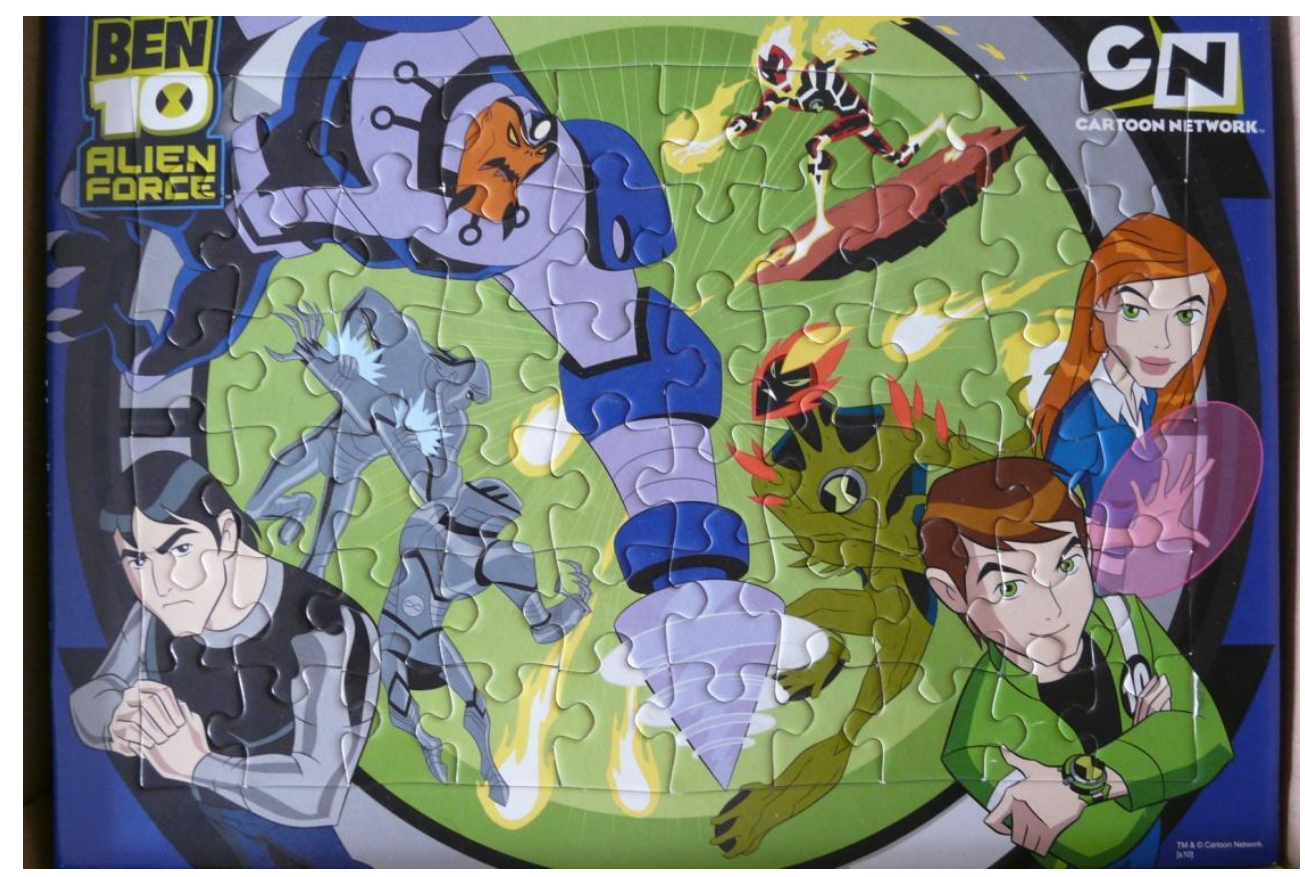

Figure 3. Photograph of the jigsaw puzzle used in the puzzle cooperation measure.

\section{Post-activity Questionnaire}

After the volunteering prosocial measure a questionnaire was administered to participants. To measure perceived similarity only the question "these people have a lot in common with me" was used from the attitude homophily scale as it captures the key component of the scale. The motivation for reducing the scale was that a number of participants in experiment 1 responded primarily in the middle of the scale for most of the attitude homophily items.

IOS targeted at other people in general was dropped, as having to compare how close participants felt to the group and other people in general in the same format might produce demand characteristic that influences their responses. Instead, the IOS targeted towards the group was used as a measure of group-specific interdependent self-construal and the Relationship-Interdependent Self-Construal (RISC; Cross, Bacon, \& Morris, 2000) was used as a measure of generalised interdependent self-construal (Cronbach alpha $=.71$ ). The RISC involves participants indicating how strongly they agree or disagree with 11 statements (2 reverse coded) about the relationship between their self and others on a 7-point likert scale from 1 (strongly disagree) to 7 (strongly agree).

As Wiltermuth and Heath found a significant difference and partial mediation with the question "how much did you feel you were on the same team with the other participants", it 
was also included as a measure of entitativity, with a 7-point Likert scale from 1 (not at all) to 7 (very much).

Participants were asked about their current mood using the UMACL (Cronbach alphas: hedonic tone $=.82$; tense arousal $=.78$, energetic arousal $=.80$ ); how well they knew each participant before the study using the same scale as in experiment 1; and two openended questions asking participants to write any general comments they would like to make about the group activity they just did and one asking for comments about the survey or the study in general. Because vocalising asynchronously might be difficult for participants, participants were also asked to rate if they though the task was difficult to do on a 7-point Likert scale from 1 (strongly disagree) to 7 (strongly agree).

\section{Results}

\section{Pre-experiment bonding}

Across all groups, 55\% of participants had never seen any of the other participants in their group before. A one-way ANOVA found no differences across conditions in how well participants knew each other beforehand using either the mean rating for each participant or the highest rating for each participant as the dependent variable $\left(F_{\max }=0.10, p=.91\right)$.

\section{Cooperation - volunteering measure}

The unrewarded study scenario appeared believable: $91 \%$ of participants volunteered for one of the four versions and only two participants gave some indication in the postactivity questionnaire that they thought it might be a test (these participants were removed from further analysis).

A one-way ANOVA with vocalising as the independent variable and cooperation as the dependent variable (the amount of time willing to donate) was conducted. The ANOVA found a significant main effect of condition: $F(2,86)=3.83, p=.03, \eta_{p}{ }^{2}=.08$. Planned comparisons between the synchrony $(M=24.00, S D=12.20)$ and asynchrony conditions $(M$ $=27.24, S D=12.22$ ) were not significant, $t(57)=1.02, p=31$, but neither was the synchrony condition significantly higher than the passive condition $(M=18.33, S D=13.15)$, $t(58)=1.73, p=.09, d=0.45$. Post-hoc examination of the difference between the 
asynchrony and passive condition was significant even with a conservative Bonferroni correction $t(57)=2.69, p=.02, d=0.71$, (see Figure 4 below).

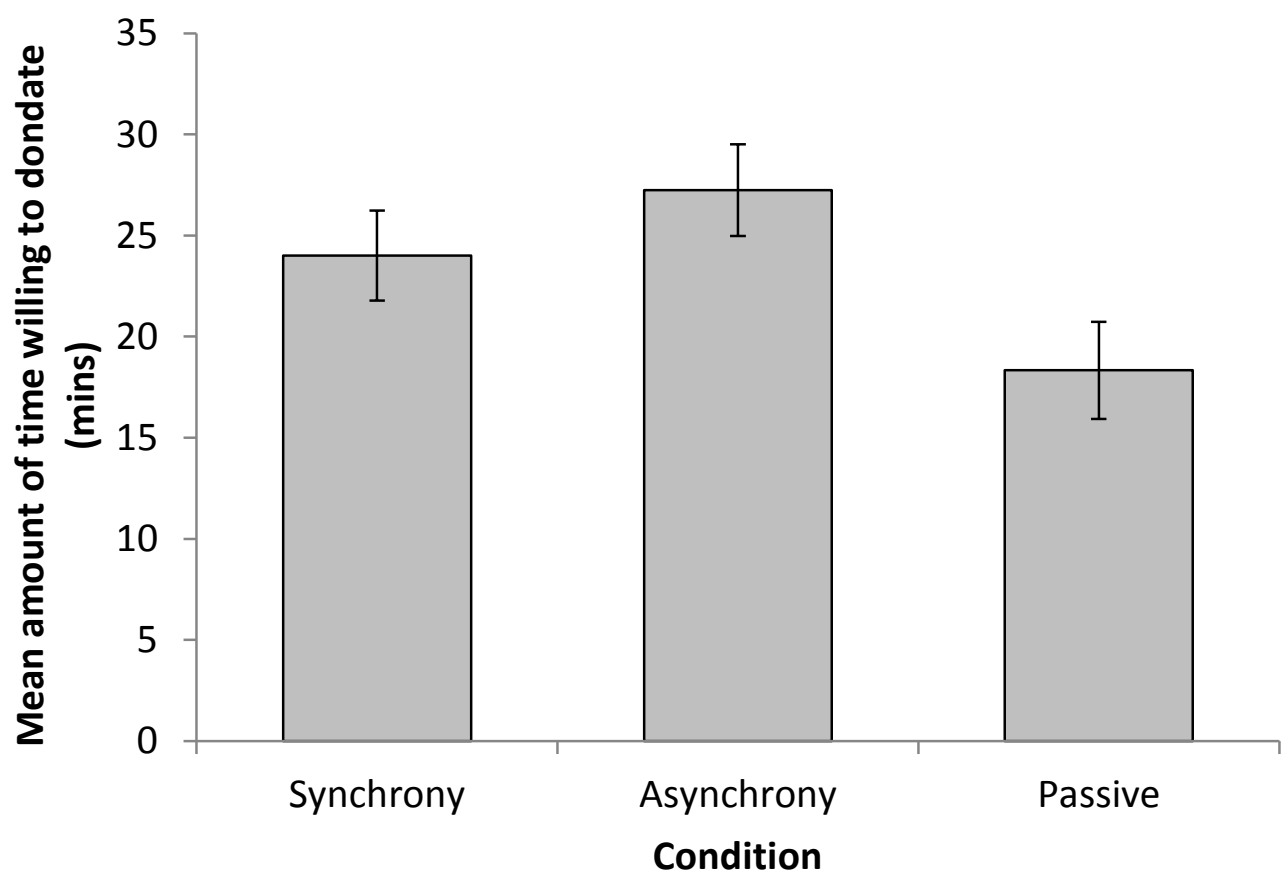

Figure 4. Mean amount of time willing to donate (minutes) for each of the vocalising conditions. Error bars are +/- SE.

Across conditions, $44 \%$ of participants who said they were willing to complete the survey actually went on-line and completed it. A lower proportion of volunteers completed the survey in the synchrony condition (36\%) than in the asynchrony (52\%) and passive condition $(46 \%)$, however, these proportions were not significantly different, $\chi^{2}(1, N=81)=$ $1.50, p=.47$.

\section{Cooperation - puzzle measure}

In terms of spontaneous helping, 16 participants helped once, 4 helped twice, and one participant helped three times. Because the number who helped more than once was small, spontaneous helping was collapsed into a dichotomous variable: helped or did not help. The overall chi-square was marginally significant, $\left.\chi^{2}(1, \mathrm{~N}=87)=5.66, p=.06\right)$. There was no difference in the distribution of helping between the synchrony ( $30 \%$ helped) and asynchrony conditions $(35 \%$ helped $),\left(\chi^{2}(1, \mathrm{~N}=56)=0.14, p=.71\right)$. However, there was a significant 
difference between the synchrony and passive condition ( $10 \%$ helped $), \chi^{2}(1, \mathrm{~N}=61)=3.99, p$ $=.05$, and between the asynchrony and passive condition, $\chi^{2}(1, \mathrm{~N}=57)=5.29, p=.02$. Odds ratios indicated that the odds of a participant helping after vocalising in synchrony were 4.00 times higher than after being passive, and the odds of helping after vocalising in asynchrony were 4.94 times higher than after being passive (see Figure 5 below).

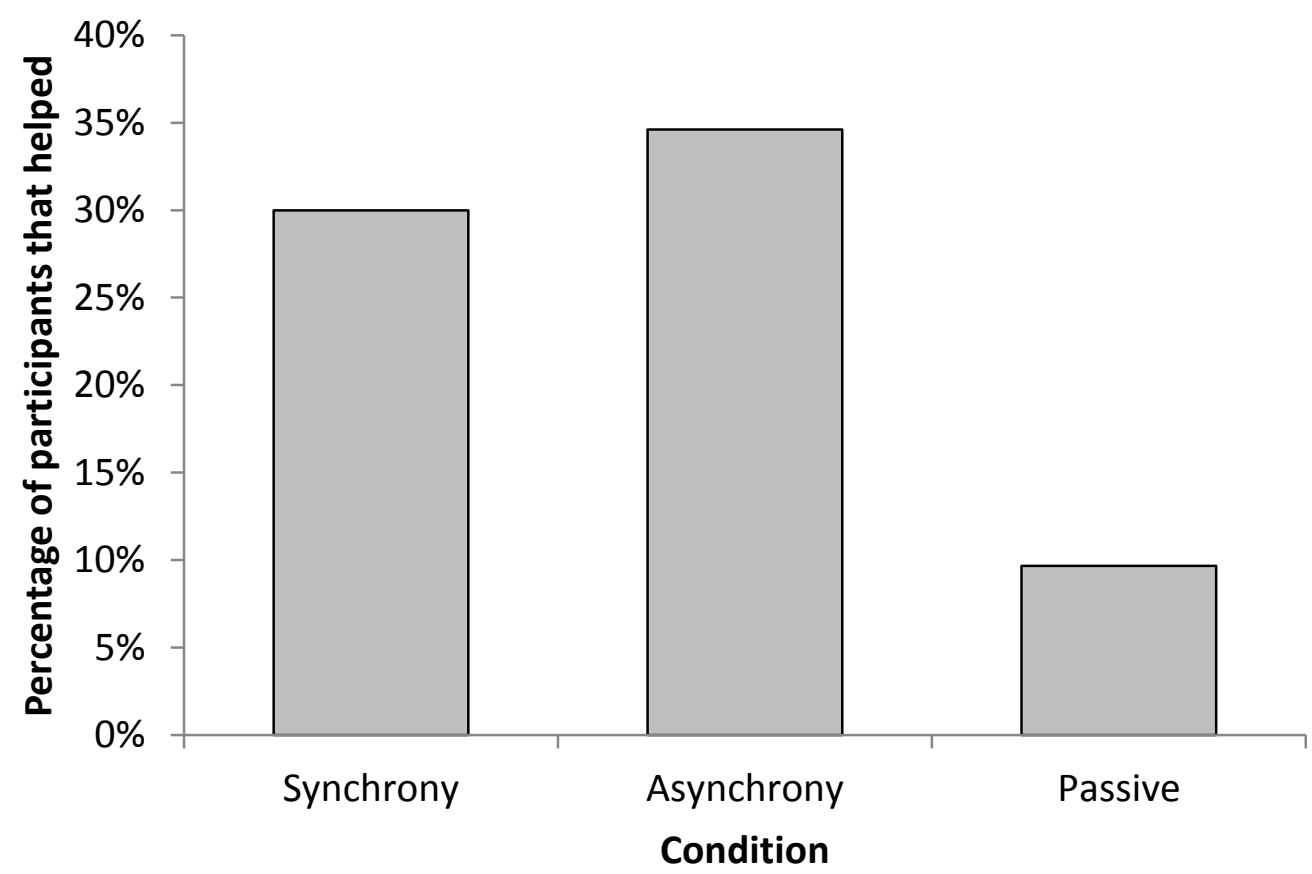

Figure 5. Percentage of participants who spontaneously helped during the puzzle task for each vocalising condition.

\section{Post-activity questionnaire}

One-way ANOVAs were performed for attitude homophily (perceived similarity), IOS and RISC (interdependent self-construal), feeling on the same team (entitativity), hedonic tone, tense arousal, and energetic arousal. No significant main effects across conditions were found for any of these self-report measures $\left(F_{\max }=1.69, p=.19\right)$.

A significant main effect was found for perceived difficulty, $F(2,86)=3.35, p=.04$, $\eta_{p}{ }^{2}=.07$. Gabriel post-hoc tests found that the asynchrony condition $(M=2.31, S D=1.65)$ had a significantly higher mean than the passive condition $(M=1.40, S D=1.13 ; p=.04, d=$ $0.64)$. The difference between the synchrony $(M=1.97, S D=1.27)$ and passive conditions 
and synchrony and asynchrony conditions were not significant $(p=.30, d=0.47$, and $p=.71$, $d=0.23$, respectively).

\section{Discussion}

The results from experiment 2 were inconsistent with the hypothesis that synchronised chanting increases cooperation. Counter to expectations, the asynchrony condition produced the highest level of cooperation for both the volunteering measure and the puzzle measure. Notably, the difference between the synchrony and asynchrony conditions was not significant, but the differences between the asynchrony and passive condition in the volunteering measure and between both of the vocalising conditions and the passive condition in the puzzle measure were significant. This indicates that it was likely some shared attribute between the synchrony and asynchrony conditions that increased cooperation compared to passive listening rather than being in synchrony. One possibility is that the slow rhythmic vocalising made participants more relaxed and in a more positive mood. However, this hypothesis is not supported by the data which found no differences with any of the mood subscales across the three conditions. Another possibility is that it is the feeling of creating a shared performance that produced greater cooperation.

Whatever may have produced the greater level of cooperation in the synchrony and asynchrony condition, the finding of greater cooperation towards both non-group members (volunteering measure) and fellow group members (puzzle measure) lends more support towards hypothesis 1a (generalised cooperation) than hypothesis 1b (group specific cooperation). However, a potential issue with the measure of cooperation towards fellow group members was that the puzzle measure was performed by the same participants after the volunteering measure and the post-activity questionnaire. The puzzle measure, therefore, was not independent from the other measures - participants' behaviour in the volunteering measure or their responses in the questionnaire may have influenced their behaviour during the puzzle measure. For example, if a participant agreed to help the confederate with the unrewarded survey, this might have made them feel good about helping (Batson, 1998) and so increased the probability that they would have helped during the puzzle activity. Therefore, in both experiment 1 and 2 there may have been confounds in the behavioural measurement of in-group cooperation (confederate influence in experiment 1; a lack of independence in experiment 2). It is possible that synchronous behaviour may produce significantly greater cooperation than asynchronous behaviour but this is only directed 
towards fellow performance group members. To explore this option, in my third experiment I only measured in-group cooperation with a commonly used economic measure: the public goods game.

\section{Experiment 3}

\section{Overview}

In experiment $3 \mathrm{I}$ repeated the same manipulation as used in experiment 1 but $\mathrm{I}$ changed the dependent variable. I predicted that participants in the synchrony condition would produce a significantly higher level of cooperation than participants in the asynchrony and no-movement conditions.

\section{Method}

\section{Participants}

Participants were 94 volunteers recruited through posters placed around Victoria University of Wellington campus (62 female; mean age $=23.08$ years, range: 17-42 years). The experiment was advertised as taking 30 minutes with participants receiving \$10 with the opportunity to earn up to $\$ 17.50$. Interested individuals were asked to e-mail for more details, with which they were sent a brief overview of what they would be asked to do (some simple body movements in a group and a short questionnaire) and were given a list of time slots they could sign up for. They were also informed that they would be given $\$ 5$ for showing up on time and an additional $\$ 5$ to play an investment game from which they could potentially earn more money. All participants were paid in cash at the end of the experiment with all participants receiving the $\$ 5$ show up fee and the accurate amount they earned from the investment game rounded to the nearest $\$ 0.10$. All groups contained exactly four participants. The distribution of females was evenly spread across conditions. ${ }^{16}$

\footnotetext{
${ }^{16} \mathrm{~A}$ chi-square analysis found no difference in the proportion of females across groups, $\chi^{2}(2, \mathrm{~N}=90)=0.69, p=$ .71 .
} 


\section{Procedure}

\section{Synchrony manipulation}

The same procedure used for manipulating synchrony in experiment 1 was also used for this experiment. Again three conditions were used: synchrony, asynchrony and passive no-movement.

\section{Public Goods Game}

Similar to Wiltermuth and Heath (2009), I used a public goods economic game to test cooperation behaviourally. I decided to use a one shot game rather than the repeated game used by Wiltermuth and Heath. Repeated games, as well as being more complicated to run, also involve reputation effects and strategies (Fischbacher, 2001; Ones \& Putterman, 2007; M. A. Spencer, Swallow, Shogren, \& List, 2009). Here I wanted to tap into participants' automatic preferences for cooperation after synchrony without additional complexity.

Participation in the game took place directly after the synchrony activity. To play the game the four participants were seated in chairs facing separate walls to minimise any communication. Participants were also told not to communicate with any of the other participants. Instructions were read out by the experimenter and also written on a sheet handed to participants so that the instructions were known to be common knowledge (Friedman \& Sunder, 1994). Participants were told they would be given $\$ 5.00$ for which they could contribute some or all of this $\$ 5$ to a group investment (detailed instructions for the game can be found in Appendix 2 on page 140). All money in the group investment would then be doubled and divided equally among all members of the group. Participants were given four different examples of potential outcomes depending on how much money people invested and also completed a quick quiz to check they understood the rules (Croson, 2005).

Participants were then handed a slip in a lettered envelope for which they could confidentially indicate how much they would like to contribute to the group investment (in $\$ 0.50$ increments). Envelopes were then collected and taken to an assistant in another room to calculate each player's earnings. Earnings were placed back in the same lettered envelope for participants to collect at the end of the experimental session. This ensured that participants' contributions remained confidential. Participants received the correct amount of money they earned (rounded to the nearest \$0.10) so there was no deception in the game. 


\section{Post-activity questionnaire}

After the economic game, participants were handed a questionnaire that had the same letter as their envelope in the economic game to allow behavioural and self-report responses to be matched. A number of the same scales used in the previous experiment were included again in the post-activity questionnaire: the UMACL to measure mood (Cronbach alpha : hedonic tone $=.75$; tense arousal $=.77$, energetic arousal $=.77$ ); the IOS targeted at the people participants just did the activity with to measure interdependent self-construal; how much participants felt on the same team to measure entitativity; perceived difficulty of the activity; how well they knew each participant before the study; and the two open ended questions asking if they had any comments about the activity or the study in general. Perceived similarity was measured with the same item Wiltermuth and Heath (2009) used in their studies: "How similar do you think you are you to the other participants?" from 1 (not at all similar) to 7 (very similar). Trust was also measured with the item: "How much did you trust the other participants going into the group investment exercise?" from 1 (did not trust at all) to 7 (trusted very much).

\section{Results}

\section{Pre-experiment bonding}

One group was removed from further analyses due to three out of the four group members indicating that they knew each other very well before the experiment. Although one member of this group was a stranger to the other three group members, all participants were removed as the group-dynamics were quite different due to the three members being very good friends. For the remainder of the 90 participants, $73 \%$ had never seen any of the other participants in their group before. One-way ANOVAs using either the mean rating for each participant or the highest rating for each participant as the dependent variable found no significant differences across conditions in how well participants knew each other beforehand $\left(F_{\max }=0.89, p=.45\right)$.

\section{Cooperation measure}

Across both conditions the amount of money placed in the investment ranged across all possible responses $(\$ 0-\$ 5)$. A histogram suggested a negative skew in particular for the 
synchrony condition, but the skewness was not significantly different from 0 (synchrony skewness $=-.79, S E=.43, z=1.85, p>.05)$.

A one-way ANOVA found no significant differences across condition for the mean amount invested in the public good (synchrony: $M=\$ 3.53, S D=1.76$; asynchrony: $M=$ $\$ 2.87, S D=1.72$; no-movement: $M=\$ 3.22, S D=1.65), F(2,87)=1.13, p=.325, \eta_{p}{ }^{2}=.05$, (see Figure 6 below).

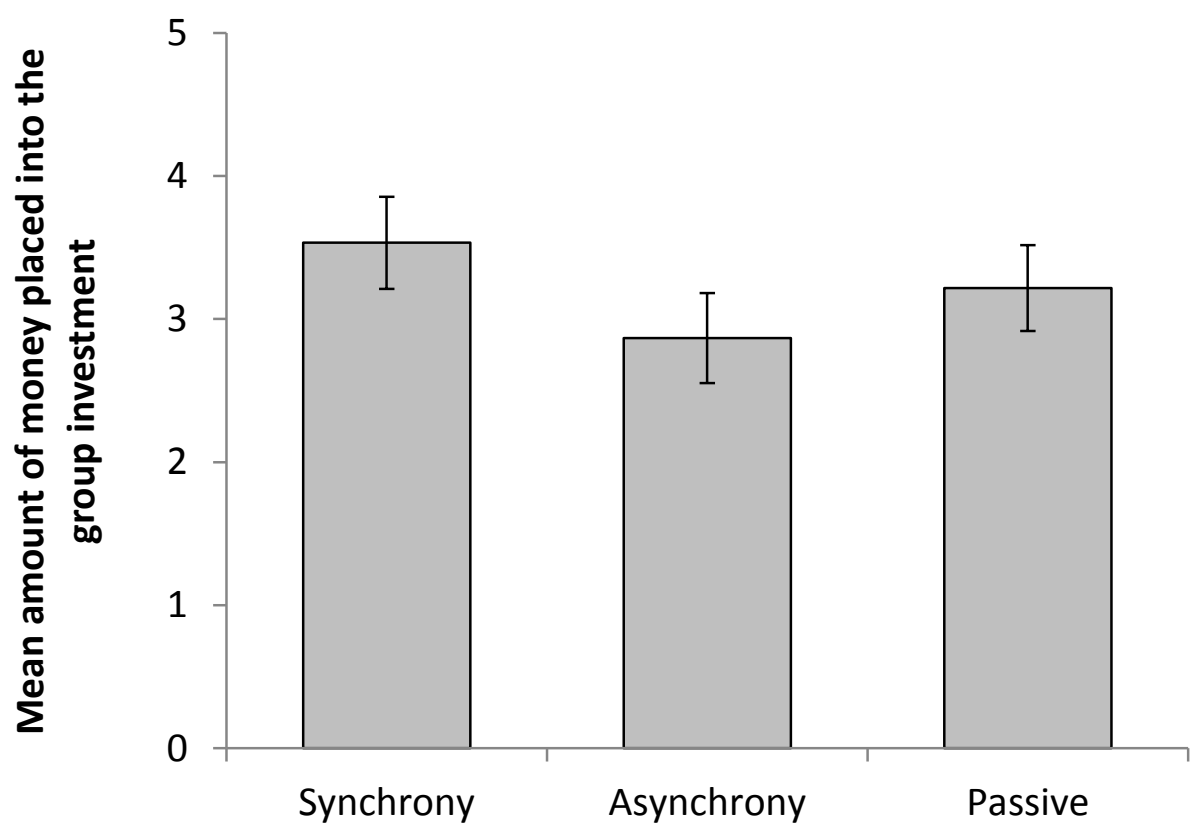

Condition

Figure 6. Mean amount of money place into the group investment (dollars) for each of the movement conditions. Error bars are +/- SE.

Even though the the differences in the means between conditions were not significant, $50 \%$ of participants in the synchrony condition put all of their money in the public good compared to $23 \%$ in the asynchrony condition. A chi-square examining the difference between conditions in the proportion of participants who invested the full $\$ 5$ was significant, $\chi^{2}(\mathrm{~N}=60,1)=4.59, p=.03$, with the odds of a participant helping after synchronous movement being 3.29 times higher than after asynchronous movement. However, the proportion who invested everything in the synchrony condition was not significantly different for the $33 \%$ who invested everything in the no-movement condition, $\chi^{2}(\mathrm{~N}=60,1)=1.71, p=.19$, with the odds of a participant cooperating after synchronous 
movement being 2.00 times higher than after passive no-movement, (see Figure 7 on page $65)$.

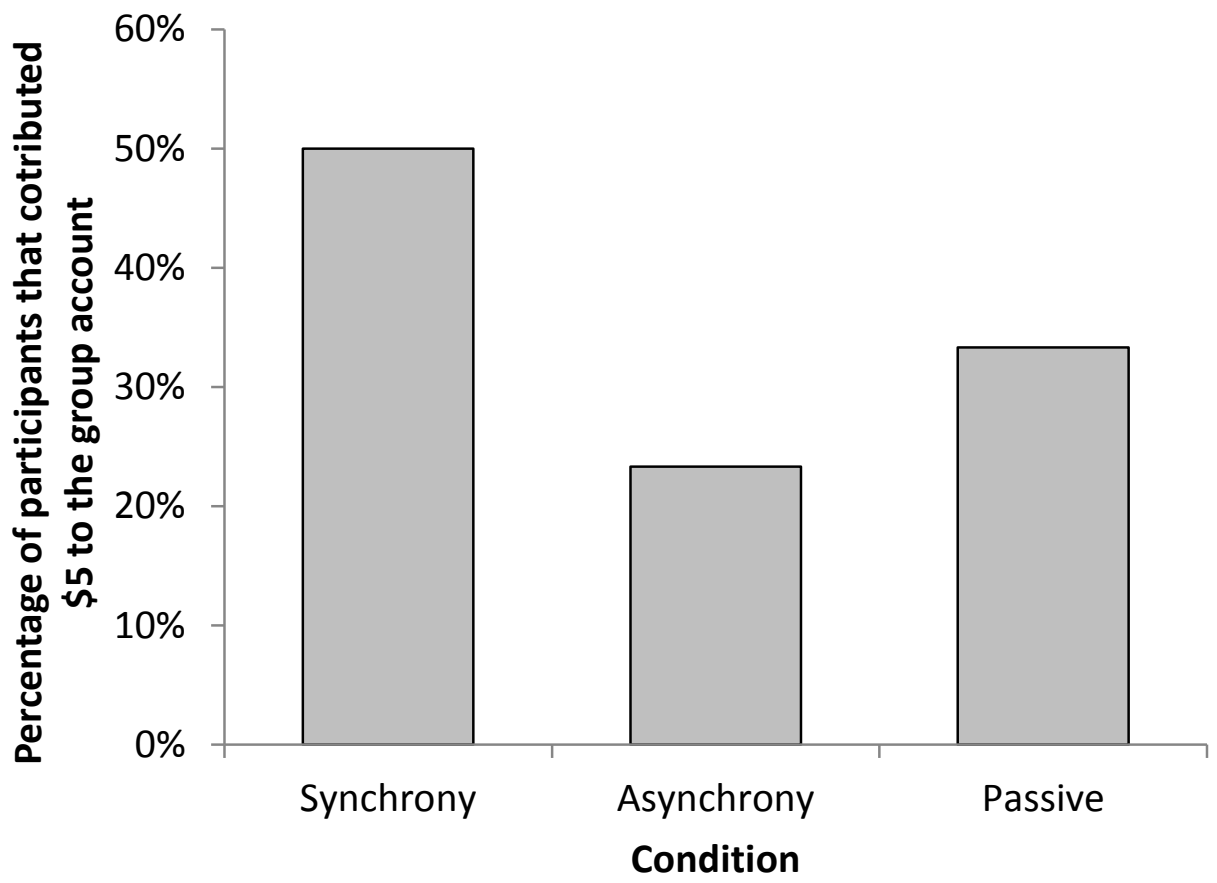

Figure 7. Percentage of participants that placed all of their money into the group account for each movement condition.

Post-activity questionnaire

One-way ANOVAs were performed for each of the self-report measures with condition as the independent variable. There were no significant differences for hedonic tone, tense arousal, energetic arousal, IOS, how similar participants felt, how much they trusted the other participants, and perceived difficulty $\left(F_{\max }=2.50, p=.09\right)$. However, there was a significant difference in entitativity, $F(2,86)=4.16, p=.02, \eta_{p}{ }^{2}=.11$. Planned comparisons between the synchrony $(M=4.78, S D=1.80)$ and asynchrony conditions $(M=4.13, S D=$ 1.71) were not significant, $t(58)=.143, p=.16, d=0.04$, but the synchrony condition was significantly higher than the passive condition $(M=3.41, S D=1.96), t(57)=2.80, p=.01, d$ $=0.74$, (see Figure 8 on page 66). Polynomial contrast analysis found that a linear trend significantly fitted the data, $F(1,86)=8.31, p<.01$. However, how much a participant felt on the same team was not correlated with how much money they invested $(r=.12, p=.26)$. 


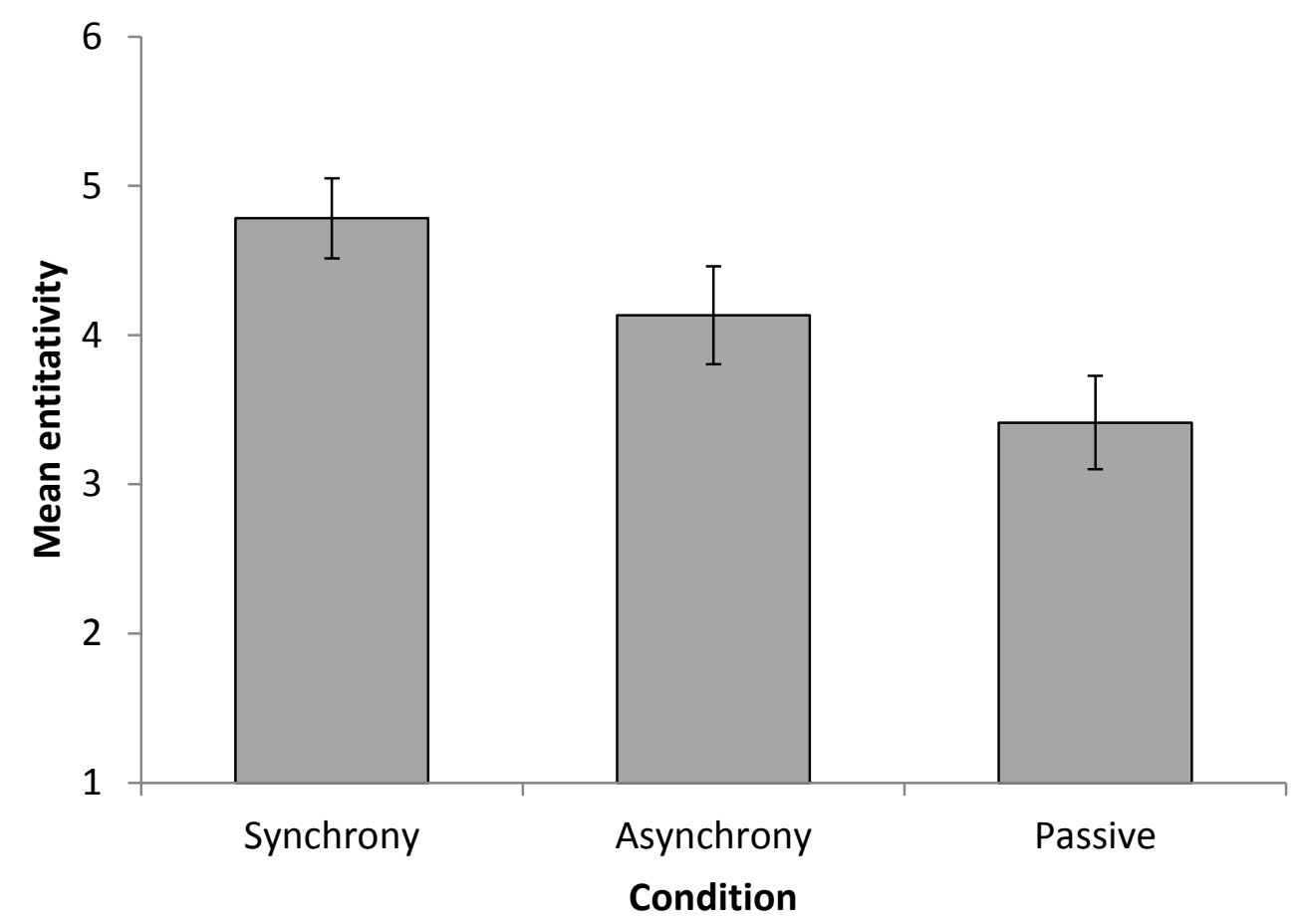

Figure 8. Mean level of entitativity for each of the movement conditions. Error bars are +/SE.

\section{Discussion}

Although I found no significant differences across the three conditions in the average amount of money given to the group investment, I did find that a significantly higher proportion of participants gave the full $\$ 5$ in the synchrony condition than in the asynchrony condition. This provides evidence supportive of the hypothesis that synchrony increases cooperation under minimal conditions. There are a couple of potential reasons why I found a difference with the proportion giving the full $\$ 5$ but not the overall mean. One possibility is that people vary in their susceptibility to synchronous activities, and under minimal circumstances only some people are affected. This could lead to an increase only at the cooperative end of the distribution rather than a shift in the whole distribution. A second possibility is the presence of a ceiling effect. Participants in the synchrony condition may have been willing to contribute more money to the group investment but could only contribute a maximum of $\$ 5$, so restricting the 'true' effect. Cooperation, even in the asynchrony condition, was quite high - participants on average were willing to give $57 \%$ of their earning to the public good, compared to around $40 \%$ in the first round of the asynchrony 
condition in Wiltermuth and Heath's study. With the $\$ 5$ ceiling, this left less room for the synchrony condition to produce a significantly higher score.

Although a lower level of cooperation was found in the passive condition compared to the synchrony condition, for both the mean amount of money given to the group investment and the proportion giving the full $\$ 5$, these differences were not statistically significant. This finding was surprising as both experiment 1 and experiment 2 found the lowest level of cooperative behaviour was with the passive condition. In the passive condition, participants watched a video of a group of four people moving in synchrony. One possibility is that observation of synchrony also increases cooperation. For example, Lakins (2010) found that participants who observe others moving in synchrony perceive those people as having greater entitativity. Perception of synchrony could prime participants to think about group cohesiveness, increasing perception of cohesiveness in their own group, and so increasing cooperation. However, the finding that entitativity was lowest in the passive group and entitativity was unrelated to cooperation does not support this particular conjecture. Moreover, as the same stimulus was used for both experiment 1 and experiment 3 it would be anticipated that both of these studies should have found similar results.

In terms of potential mediating factors, a significant main effect of entitativity was found with a significant linear effect across the three conditions. This could suggest that synchrony influences group identity potentially leading to an increase in the salience of group-level self-categorisation. However, entitativity was not related to cooperation so it is unclear what role, if any, entitativity may play in increasing cooperation with synchronous movement.

\section{Chapter Discussion}

Both experiment 1 and experiment 3 found evidence consistent with the hypothesis that synchrony produces greater cooperative behaviour. In each of these experiments synchrony produced the greatest level of cooperation. Although, in experiment 1 the difference between the synchrony and asynchrony condition was not significant, the significant linear trend across the conditions supported synchronous movement having a cooperative effect, with asynchronous movement showing intermediate levels of cooperation. Moreover, in experiment 3, a significantly higher level of cooperation was found after synchronous movement compared to the asynchronous movement. This suggests that 
synchrony can increase cooperation even when other social, emotional, and cultural factors are minimised.

Experiment 2, on the other hand, found no evidence of synchronous vocalising having an effect compared to asynchronous vocalising. This suggests that synchronised vocalising does not affect cooperation under minimal conditions. One possibility for why no synchrony effect was found in experiment 2 was due to the way synchrony was created. In the experiments in this chapter, synchrony was artificially and experimentally manipulated through an external pulse so as to standardise the movements and vocalisations. However, many social interactions require purposeful coordination by the actors, i.e., synchrony is created by the performers. In the next chapter I explore the possibility that working together to create synchrony produces large more consistent synchrony-cooperation effects. 


\section{Chapter 5 \\ SYNCHRONY AND SHARED INTENTIONALITY}

\section{Shared intentionality in music and dance}

Chapter 4 explored the idea that a person merely perceiving others acting in synchrony with them is sufficient to increase their tendency to cooperate. Although I found some evidence in support of this hypothesis, the effect was small and not replicated with chanting. This chapter examines if larger and more reliable increases in cooperation can be created by making the synchrony manipulation more realistic for how group synchrony is created in natural ecologies. Specifically, I examined the effect of creating synchrony through shared intentionality (Tomasello \& Carpenter, 2007; Tomasello et al., 2005).

To manipulate synchrony in chapter 4 , participants were asked to move or chant in time with their own beat playing through their headphones - a rather individualistic goal. Synchrony was created incidentally, as participants were directed to attune to the same metronome beat. Although synchrony created by individuals entraining to a shared recorded beat can be observed in the real world - for example in modern dance clubs - most group music and dance is the outcome of teamwork (Lomax, 1968). By teamwork, I mean that participants have a shared goal of creating a music and/or dance performance, a shared action plan of how to obtain that goal, and shared attention focused on the group's unfolding performance. The sharing of such psychological states to produce collaborative behaviours is known as shared intentionality. As discussed in chapter 2 (see page 10), the shared goal of most performances of group music and dance is the creation of rhythmic synchrony (Kirschner \& Tomasello, 2009, 2010). Entrainment during music and dance is not accidental, nor is it an unintended consequence of interaction with like-minded conspecifics. Music and dance is a deliberately communal practice. Performers intentionally modify the timing of their rhythmic movement to entrain to the behaviour of others with the knowledge that others also share the goal of entrainment (what I term intentional group synchrony). Performers are aware that only by working together will they achieve their shared goal of acting in synchrony. By using shared intentionality to create synchrony, performers create cognitive coordination as well as body coordination. 
Kirschner and Tomasello (2010) suggest that the use of shared intentionality to create group music and dance could be a key proximal mechanism for the generation of music's cooperative effects. One possibility is that the process of sharing intentionality facilitates future cooperation independently of whether synchrony is involved or not. Such an effect could be mediated by psychological variables including interdependent self-construal, entitativity and perceived similarity. For example, creating shared intentionality involves cognitively representing and integrating another person's mental states with one's own (Tomasello et al., 2005). Sharing mental states could lead to a broader integration of the self with other, thereby producing a more interdependent self-construal. Working together to achieve a shared goal could induce feelings of common fate - that is the outcome of their endeavour is dependent on each other - with shared fate being found to increase feelings of entitativity (Castano, Yzerbyt, \& Bourguignon, 2003). Furthermore, believing that one shares mental states with others could also plausibly increase perceived similarity.

\section{Synchrony and shared intentionality}

Another possibility is that shared intentionality and synchrony could combine to foster cooperation to a greater extent than either synchrony or shared intentionality alone. Here, I suggest two plausible psychological mechanisms by which synchrony could combine with shared intentionality: (1) increased attention directed towards the other participants; and (2) reinforcement of cooperation.

\section{Increased attention}

Shared intentionality usually involves joint attention (Tomasello et al., 2005). This is particularly the case when people are working together to create synchrony - performers need to be constantly paying attention to the other performers' behaviour to check that they are in time. If synchrony increases cooperation through perception-action links or through entitativity (as described in chapter 4), increased attention directed towards the behaviour of others moving in synchrony could increase the effect of joint synchrony. For example, the more time a performer observes another performer moving in the same way at the same time, the greater the degree of self-other blurring. This theory could also explain why small synchrony-cooperation effects were found in experiments 1-3: when there is no shared intentionality participants had little motivation to pay attention to the other participants. 


\section{Reinforcement of cooperation}

As described in chapter 2, Hagen and Bryant (2003) suggest that music and dance were selected because they provided a signal for other groups that the performing group is effective at acting collaboratively to achieve joint goals. Groups who effectively sing and dance together can effectively fight together. Such a signal through music and dance could ward off potential threatening groups, and attract potential allies.

A compatible alternative to the Hagen and Bryant hypothesis is that music and dance might (also) provide an internal signal for those performing the dance that they are effectively collaborating together. As mentioned above, working together towards a common goal could increase feelings that the collaborators are a team. However, such an effect may be dependent on whether the goal is perceived to have been successfully achieved. If a number of people work together to achieve a shared goal but fail to achieve that goal, this could result in reduced confidence in the other actors and in turn reduced cooperation. Notably, for many collaborative tasks success is not guaranteed. On a collective hunt, for example, success of actually catching an animal may be unpredictable. Furthermore, it may be difficult to assess everybody's contribution - if an animal is caught it may have been due to the efforts of only a few people, not everybody involved in the hunt. The others in the hunting group may have put little effort into it. What might be needed for cooperation to be reliably fostered through shared intentionality is a group activity in which there is a high probability of achieving the goal, in which people can easily monitor others to assess their contribution, and in which success can be easily determined. Intentional group synchrony would appear an ideal candidate for such cooperation reinforcement. Working together to attain synchrony has a very high likelihood of success - people the world over appear very competent at creating group synchrony. Because a performance of intentional group synchrony involves people coming together to the same place at the same time, it is often easy to observe everybody's contribution as the performance is unfolding before everyone's eyes. If somebody in the group stops trying to entrain their movement to the others, participants will quickly fall out of time. Because the margin of error for synchrony is so small the other participants will soon notice that one of the participants is not pulling their weight. Moreover, the observation of synchrony provides instantaneous feedback that participants are working successfully together. Each time they repeat a rhythmic action in concert, it tells all members of the group they are effectively cooperating. As the action is repeated again and again, performers receive a consistent message that the group can cooperate together. Perceiving that everybody was 
successful at achieving their shared goal of synchrony intensifies the feeling that such a result has been achieved together, which in turn increases feelings of entitativity. Subsequently, such increased feelings of entitativity could lead to a shift in interdependent self-construal or greater feelings of similarity. Such psychological effects might in turn increase cooperation through trust or through other psychological mechanisms described in chapter 3 (such as empathy and social norms).

\section{Current studies}

The primary aim of the studies in this chapter was to examine if synchrony created through shared intentionality produced a larger and more reliable effect than synchrony that was incidental. Shared intentionality was created by giving participants an explicit shared goal. Experiment 3b, was a continuation of experiment 3. I ran an additional condition involving participants intentionally moving together in synchrony and compared this new condition to the previous conditions. Experiment 4 examined the effect of using shared intentionality to produce synchronous vocalisations. This condition was compared against a condition in which participants intentionally chanted out of phase with each other. Finally, in experiment 5, shared intentionality was manipulated along with synchrony so that the interaction between synchrony and shared intentionality could be fully explored.

\section{Experiment 3b}

\section{Overview}

As an initial test of the effect of using shared intentionality to create synchrony, a new condition was trialled that involved participants intentionally moving in synchrony. This condition was run directly after experiment 3 . I hypothesised that combining synchrony with a shared goal would produce significantly higher cooperation than observed in the previous conditions which did not include a shared goal. I also hypothesised that the shared intentionality condition would produce significantly greater levels of entitativity, perceived similarity and interdependent self-construal when compared to each of the other conditions which lacked shared goals. 


\section{Method}

An additional 29 participants were recruited through the same method described in experiment 3 ( 23 female; mean age $=23.31$ years, range: $18-38$ years). The procedure of the new shared goal condition was exactly the same as the synchrony condition in experiment 3 except participants were not played a metronome beat with which to keep time. Instead, they were explicitly told that the goal of the task was to move in time with each other. Participants were given a brief 10 second practice to check that everyone was able to perform the movements in time. They were told when to start and when to stop, and after each two minute interval to change movement (arm swinging, body swaying, and stepping).

\section{Results}

\section{Manipulation check}

From visual observation participants appeared able to be able to effectively keep in time with each other with no metronome beat to guide them. Participants did not rate working together to create synchrony in the shared goal condition $(M=1.59, S D=1.24)$ any more difficult than keeping in time with the metronome beat in the synchrony condition $(M=1.63$, $S D=0.89), t(57)=0.17, p=.87$.

\section{Pre-experiment bonding}

For the participants in the new shared goal condition, $69 \%$ of participants were complete strangers. Independent t-tests ${ }^{17}$ comparing the shared goal condition to each of the other conditions found that there was no differences in how well participants knew each other before the study using either the mean rating for each participant or the highest rating for each participant as the DV, $t_{\max }=.91, p=.37$.

\footnotetext{
${ }^{17}$ Because I was only interested in the effect of the new shared goal condition, planned contrasts comparing this condition to the original three conditions were performed.
} 
The mean amount of money contributed to the public good for the new condition was $\$ 3.88(S D=1.59)$, (see Figure 9 on page 74 ). Similar to the original synchrony condition of experiment 3 , the data was strongly negatively skewed, with $55 \%$ of participants choosing to place the full $\$ 5$ into the group investment (see Figure 10 on page 75). As this skewness was highly significant (skewness $=-1.31, S E=.43, z=-3.05, p<.01$ ) to compare this new condition to the original conditions in experiment 3, non-parametric statistics were used. Mann-Whitney $U$ tests found that the level of investment in the shared goal condition $(M d n=$ $\$ 5.00)$ was significantly higher than the asynchrony condition $(M d n=\$ 3.25), U=277.50, z=$ $-2.47, p=.01, r=.32$, but was not significantly higher than the synchrony condition $(M d n=$ $\$ 4.50), U=395.00, z=-.66, p=.51, r=.09$, nor the passive condition $(M d n=\$ 2.75), U=$ $329.50, z=-1.68, p=.09, r=.22$.

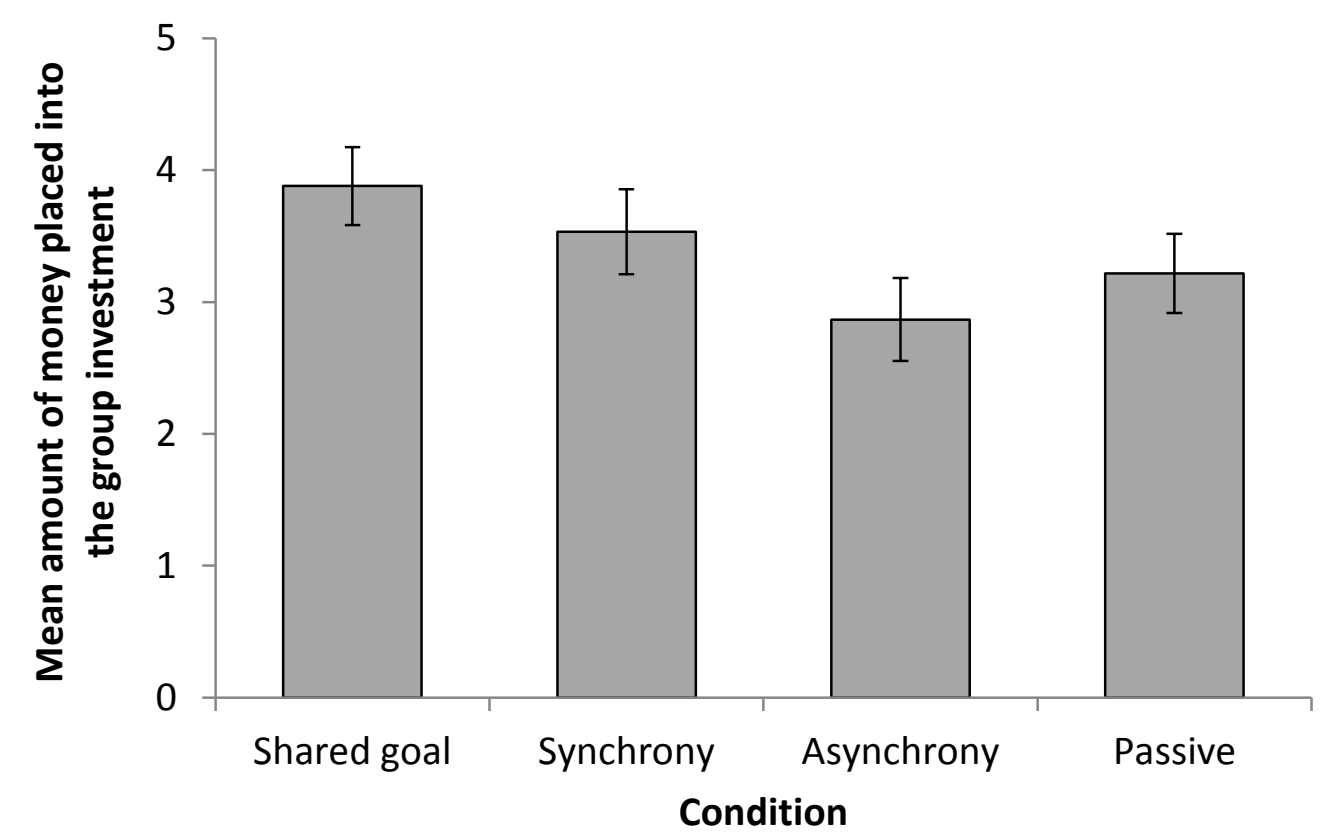

Figure 9. Mean amount of money placed into the group investment (dollars) for the new shared goal condition compared to the original three conditions from experiment 3. Error bars are +/- SE. 


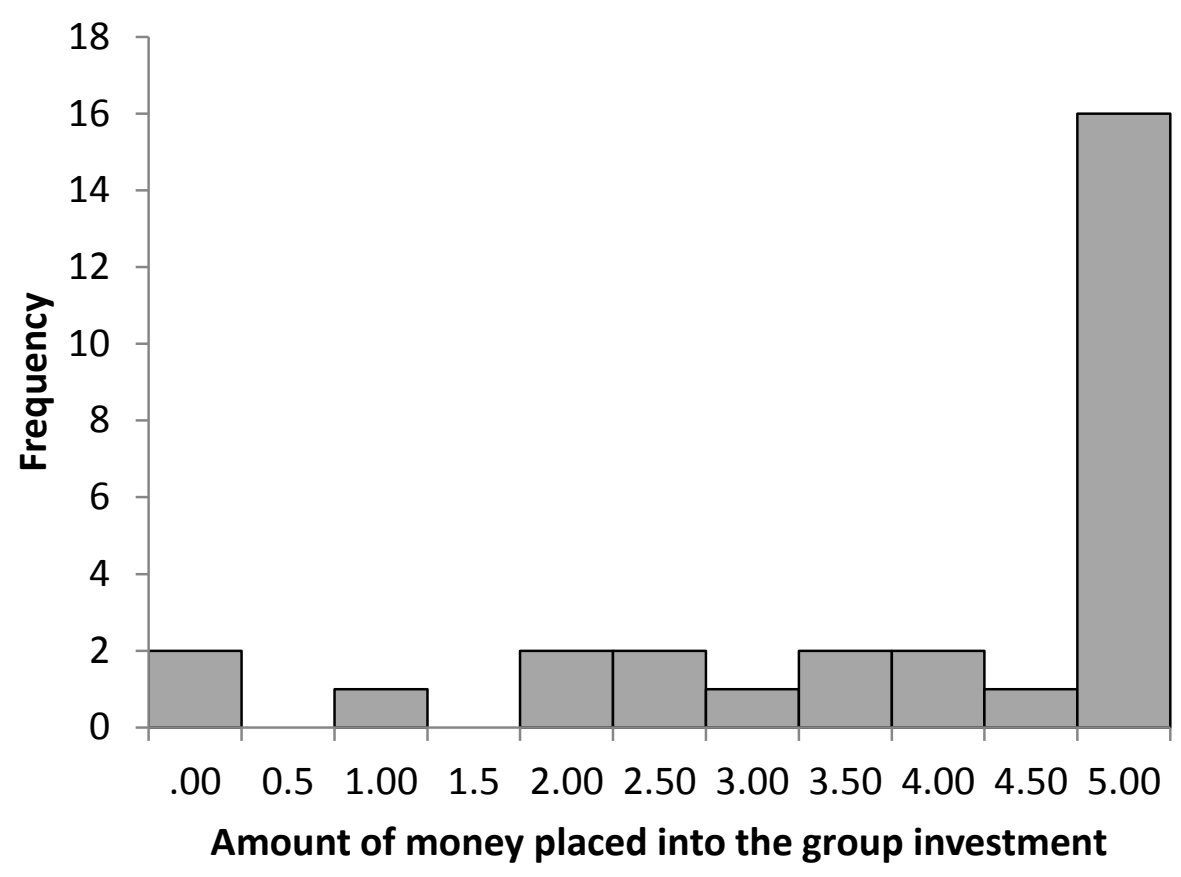

Figure 10. Histogram of the distribution of the amount of money (dollars) placed into the group investment for the shared goal condition.

The proportion of participants who donated the full $\$ 5$ in the shared goal condition was also compared to the other original conditions (see Figure 11 on page 76). Significantly more participants contributed everything in the shared goal condition than in the asynchrony condition, $\chi^{2}(\mathrm{~N}=59,1)=6.28, p=.01$, the odds of a participant helping after synchronous movement were 4.04 times higher than after asynchronous movement. However, the proportion who donated the full $\$ 5$ in the shared goal condition was not significantly different for the synchrony condition, $\chi^{2}(\mathrm{~N}=59,1)=.16, p=.69$, or the passive condition, $\chi^{2}(\mathrm{~N}=59$, 1) $=2.85, p=.09$, with odds ratios of 1.23 and 2.46 respectively.

\section{Post-activity questionnaire}

Interdependent self-construal, as measured by the IOS scale, was significantly higher in the shared goal condition $(M=3.69, S D=1.71)$ than in each of the other three conditions: versus synchrony $(M=2.47, S D=1.25), t(57)=3.14, p<.01, d=.81$; versus asynchrony $(M$ $=2.47, S D=1.43), t(57)=2.97, p<.01, d=.77$; versus passive $(M=2.28, S D=1.25), t(56)$ $=3.59, p<.01, d=.94$, (see Figure 12 on page 76 ) 


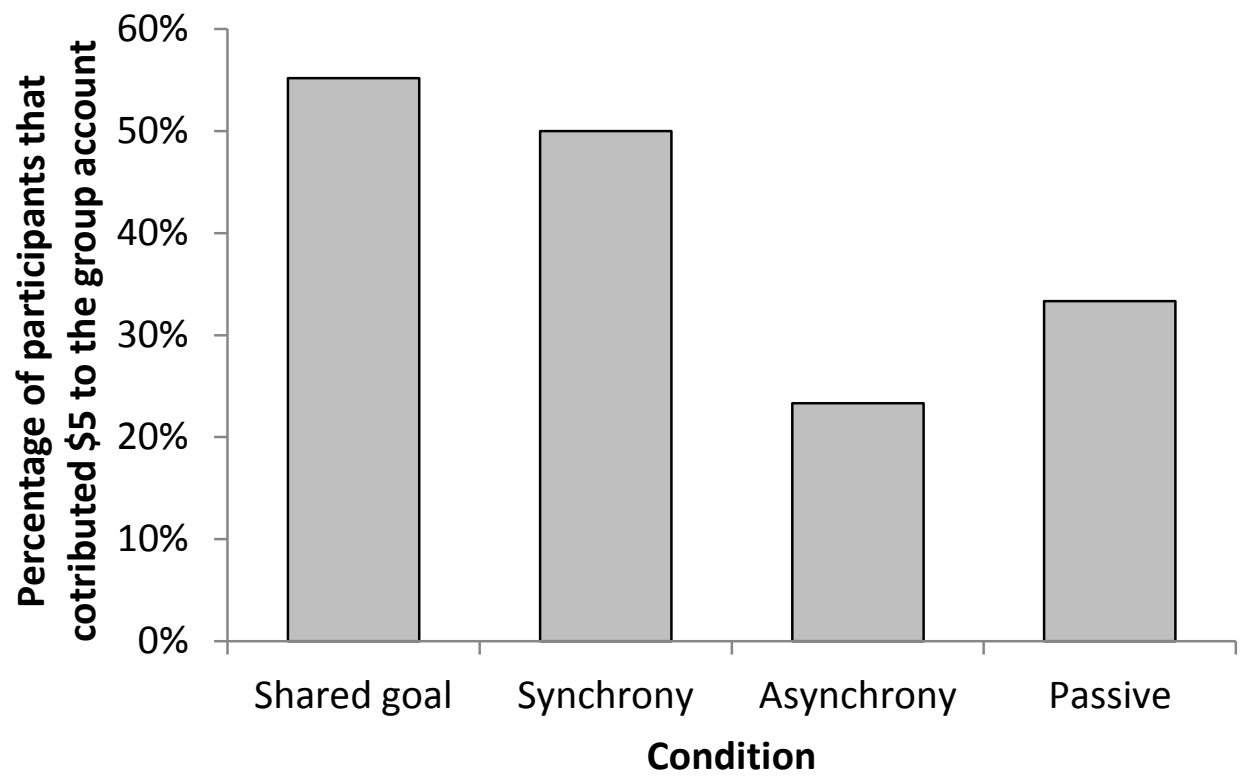

Figure 11. Percentage of participants that placed all of their money into the group account for the new shared goal condition compared to the original three conditions from experiment 3.

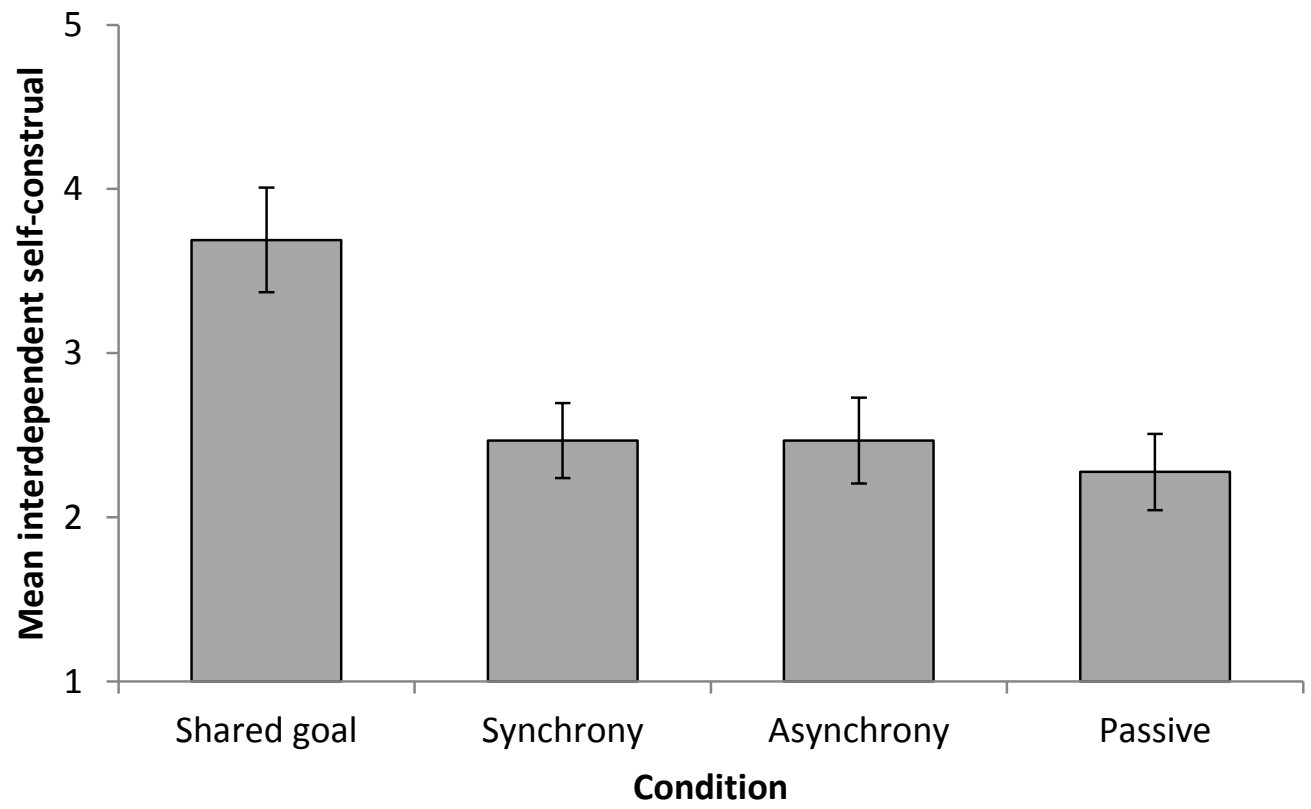

Figure 12. Mean interdependent self-construal for the new shared goal condition compared to the original three conditions from experiment 3. Error bars are $+/-\mathrm{SE}$.

The mean level of entitativity for the shared goal condition $(M=4.90, S D=1.45)$ was only significant higher than the passive condition $(M=3.41, S D=1.96), t(56)=3.28, p<$ $.01, d=.94$. The difference between the shared goal and asynchrony conditions $(M=4.13$, 
$S D=1.71)$ was marginally non-significant, $t(57)=1.84, p=.07, d=.49$. The difference between the shared goal and the synchrony condition was not significant $(M=4.78, S D=$ 1.80), $t(57)=0.27, p=.79, d=.07$.

The mean level of similarity for shared goal condition $(M=4.24, S D=1.66)$ was only significantly higher than the passive condition $(M=3.34, S D=1.32), t(56)=2.28, p=.03, d$ $=.60$. Both the differences between the shared goal and the asynchrony conditions $(M=3.80$, $S D=1.47), t(57)=1.08, p=.28, d=.28$, and the shared goal and the synchrony conditions $(M=3.77, S D=1.52), t(57)=1.14, p=.26, d=.30$, were not significant.

Even with the inclusion of the new shared goal condition, entitativity and similarity were not significantly correlated with the amount of money participants placed into the group investment ( $r=.13, p=.18$ and $r=.06, p=.55$ respectively). However, interdependent selfconstrual was marginally significantly correlated with the money placed into the group investment $(r=.18, p=.05)$, and a point-biserial correlation between interdependent selfconstrual and whether or not a participant put in the full $\$ 5$ was significant $\left(r_{\mathrm{pb}}=.20, p=.03\right)$.

\section{Discussion}

This first trial of using shared intentionality to produce synchrony provided some encouraging results. The shared goal condition produced a slightly higher level of cooperation than the other three previous conditions - although the degree of cooperation in the shared goal condition was not significantly greater than the synchrony condition and the passive condition. However, as suggested in chapter 4, this cooperation measure may have been influenced by a ceiling effect. Certainly, the significant negative skew in the shared goal condition with the majority of participants (55\%) choosing the full $\$ 5$ would support this interpretation of the results.

More impressive were the large differences across the experimental conditions observed for interdependent self-construal. Participants in the shared goal condition felt significantly closer to their fellow performance group members than compared to all of the other conditions. This finding supports the idea that working together to create synchrony results in participants seeing their self as more integrated with the group.

This first experiment introducing shared goals, therefore, provided some evidence in support of shared intentionality increasing the synchrony-cooperation effect. However, the greater cooperation and self-construal seen in the shared goal condition might have arisen solely from shared intentionality - being in synchrony may have contributed little or nothing 
on top of shared intentionality to modulate cooperative cognition and behaviour. Indeed, working together to move out of time with each other might also increase cooperation. To examine this possibility, experiment 4 compared the cooperative effects of intentional synchrony to the cooperative effects of intentional asynchrony.

\section{Experiment 4}

\section{Introduction}

Experiment 4 evaluated whether intentional synchrony produces greater cooperation than intentional asynchrony. To manipulate synchrony, chanting was used instead of movement. Recall that experiment 2 found no evidence that synchronous chanting produced greater cooperation relative to the chanting out of time. Indeed, descriptive statistics revealed that cooperation in the asynchrony condition was slightly higher than in the synchrony condition - although analysis revealed no significantly statistical difference. If intentional synchronous chanting were to produce a significantly higher mean than intentional asynchronous chanting this finding would offer impressive evidence that shared intentionality combines with synchrony to influence future cooperative tendencies.

An initial challenge to exploring the interaction of synchrony and shared intentionality came from selecting an appropriate control condition. Recall that in experiment 2, participants found chanting at different speeds significantly more difficult than chanting at the same speed (see page 59). I ran a small trial to determine whether participants could intentionally chant in groups of three at different speeds without a guiding beat. Results were not encouraging. The interference from the vocalisations of others was too great for participants to maintain their own rhythmic chanting. This was the case even with guidance from the experimenter specifying who should speed up and who should slow down if they found themselves in time. Either participants ended up matching in time, or their chanting would break down, sometimes punctuated by expletives of frustration.

Rather than comparing synchronous chanting to chanting at different speeds (i.e., manipulating frequency-locked synchrony), a new control condition was devised in which participants moved out of phase with each other (i.e., manipulating phase-locked synchrony). Although no study has yet examined the impact of relative phase on cooperative behavior, several studies have examined the effect of phase on social cognition. Both Macrae, Duffy, Miles, and Lawrence (2008) and Miles, Nind, Henderson, \& Macrae (2010) found that 
participants who moved in-phase remembered more information about another participant than participants who moved anti-phase ( $180^{\circ}$ out of time).

Testing anti-phase with groups is not possible, as when there are more than two people moving anti-phase at least two people would always end up moving in-phase with each other as a by-product of moving anti-phase to a common target. To create a manipulation in which everybody would be out of phase with each other, participants were asked to move one after another in a systematic rhythmic way - that is, they moved sequentially.

For this experiment, the passive condition was dropped, as creating a passive condition in which there was also a shared goal proved difficult. However, because my key interest was to determine whether there was a significant difference between the synchrony and asynchrony conditions the lack of a passive control condition was not of critical importance to this study.

To test cooperation I used a different economic game from the public goods game. The decision to shift games was driven by two main motivations: (1) concerns over confusion on how to play the game, and (2) whether the prisoner's dilemma, for which the public goods game is a multi-player version, is the best model for cooperation. Confusion has been cited as a potential confound in the public goods game (e.g., Andreoni, 1995), with confusion being found to explain a sizeable proportion of the level of contribution, especially in early rounds of repeated games (Houser \& Kurzban, 2002). Certainly, the instructions in experiment 3 to explain the game were long (see Appendix 2 on page 140). One participant in the study did explicitly say after the experiment they didn't understand the game and another participant wrote in the post-activity questionnaire that "the gaming part requires quite some thinking". What I really needed was a game in which participants didn't have to think what the most rational option was.

A number of theorists have questioned whether the prisoner's dilemma is the best or most common model for cooperation (Binmore, 1998; Bulbulia, 2012; Calcott, 2008; Schelling, 1960, 1973; Skyrms, 2004). Although scenarios in which cheating is an issue do obviously exist, in many forms of cooperation mutual cooperation is actually the most desirable outcome and maximises the benefits of all involved. To model such cooperation problems where social coordination is a problem that involves risk (but not necessarily defection), economists use the stag hunt, a game inspired by a passage in Rousseau's (1754) Discourse on Inequality: 
If a deer was to be taken, every one saw that, in order to succeed, he must abide faithfully by his post: but if a hare happened to come within the reach of any one of them, it is not to be doubted that he pursued it without scruple, and, having seized his prey, cared very little, if by so doing he caused his companions to miss theirs. (If9)

What Rousseau suggests is that everybody would do best by hunting the deer. However, there is always the risk that one of the hunters will go for the no-risk but lower stake option of the hare. In the stag hunt there are two equilibriums - a pay-off dominant equilibrium in which players are attracted to the larger pay-out of mutual cooperation (the deer); and a risk-dominant equilibrium in which players are attracted to the pay-out that will maximise their money irrespective of what the other may decide (the hare). What a player chooses depends on his/her expectations about what others will choose. The stag hunt is therefore simply a test of social prediction. Everybody wants to cooperate, but do they think the other players will cooperate as well? The key difference between the prisoner's dilemma and the stag hunt, then, is that in the prisoner's dilemma defection is the dominant strategy, whereas in a Stag Hunt there are two equilibria, all cooperate and all defect. All would prefer the cooperation equilibrium because it pays best. Yet if one loses nerve and selects the safe option, so should all. ${ }^{18}$

We see stag hunts in collective hunting, but we also see stag-hunts in mundane conventions of ordinary life, for example in coordinating on which side of the road to drive (Bulbulia, 2012). Skyrms (2004) argues that even when a one-off cooperation problem can be modeled as a prisoner's dilemmas, over multiple games with the same participants this problem turns into a stag-hunt. If one adopts a strategy of always defecting, although initially this may pay better when playing with conditional cooperators, ${ }^{19}$ other players will learn not to cooperate with the compulsive defector. The defector will end up only ever obtaining the certain but lower paying mutual defection option. Those who are conditional co-operators, on the other hand, will eventually reach a pay-off dominant equilibrium - the higher risk (there is always a chance someone will change strategy) but higher paying mutual cooperation option.

Wiltermuth and Heath used a variant of the Stag-hunt in their second study - the weakest link game (see page 28). However, the matrix of choices that Wiltermuth and Heath

\footnotetext{
${ }^{18}$ The classic stag hunt requires full participation, however coordination problems with risk also arise when less than full cooperation is needed to secure the benefit (Binmore, 2008)

${ }^{19}$ Conditional co-operators are those who cooperate with others with a past history of cooperating but defect with those with a past history of defecting.
} 
used was complex and could have proved difficult for participants to understand. I instead decided to use the game as a binary choice: cooperate or defect. There is a downside to using a binary choice: a dichotomous dependent variable reduces variance, which meant I could not assess the degree to which participants were willing to cooperate. However, the benefit of this choice was that it resulted in a simple decision problem, with few instructions, that should be easy to understand and interpret. Furthermore, a binary choice provides a suitable model for many cooperative situations. For example, you join the Union or you do not; you trust that the airport mechanics have done their job and so board the plane, or you do not; you trust that your friends will meet you at an agreed location, or you do not. While many important cooperation problems do involve degrees of investment in a cooperative outcome, it is important to develop appropriate psychological measures for those that do not.

In summary, in experiment 4 , the cooperative tendencies of participants who had intentionally vocalised in-phase (synchrony condition) were compared against the cooperative tendencies of participants who had intentionally vocalised sequentially (sequential condition). It was hypothesised that a greater proportion of participants vocalising in synchrony will choose the cooperative option on the stag hunt game than those who chanted sequentially. To help elucidate the psychological mechanism behind the effect, the experiment again measured similarity, entitativity and interdependent self-construal. It was hypothesised that the mean of each of these constructs would be higher in the synchrony condition than in the sequential condition. Because the stag-hunt game taps into how much participants trust the other participants to cooperate, self-reported trust was measured. The degree of trust was predicted to be higher in the synchrony condition with trust predicting cooperative behaviour. Three new scales were created to measure perceived synchrony, perceived cooperation, and attention directed towards the other participants. The degree of perceived synchrony operated as a manipulation check - it is predicted that participants in the synchrony condition should rate their activity as significantly more synchronous than those participants who chanted sequentially. If any difference in cooperative behaviour between the two conditions was caused by synchrony being used as a cue for successful collaboration, it would be predicted that the synchrony condition would have a higher mean level of perceived cooperation. If any difference in cooperation between the conditions is caused by the greater attention directed at the other participants, it would be predicted that the synchrony condition would result in a greater level of attention directed at the other participants. 


\section{Method}

\section{Participants}

Participants were 27 volunteers assigned to groups of three. Participants were recruited through posters placed around Victoria University of Wellington campus advertising a paid psychology study (18 female; mean age $=22.23$, range: $18-58$ years). The experiment was advertised as taking 30 minutes with participants receiving $\$ 12$ with the opportunity to earn more. A website was created containing additional information for interested individuals to sign up for experimental sessions. Participants were paid in cash at the end of the experiment. All participants received the $\$ 5$ show up fee with some participants receiving an additional $\$ 7$ or $\$ 10$ depending on the outcome of the economic game. The distribution of females was evenly spread across conditions. ${ }^{20}$

\section{Procedure}

A between subject design with two conditions of vocalising (synchrony and sequential) was used. Participants initially performed the chanting activity, then played the economic game and finally completed the post-activity questionnaire.

\section{Synchrony manipulation}

For the chanting activity, participants sat in chairs in a row facing a blank wall. Participants chanted 54 of the emotionally neutral words selected in experiment 2 from the Affective Norms for English Words database (Bradley \& Lang, 1999). ${ }^{21}$ Twelve pages of the words in random order were created for participants to read, with all 54 words listed on every page in three columns. Participants read the words until the 6 minute activity was over. In the synchrony conditions all the participants in a group read the same words. In the sequential condition each participant read the words from only one column.

Participants were given headphones through which a metronome beat was played through a portable digital audio player to help participants initially coordinate with each other. All participants heard the same metronome beat via a four-way headphone splitter.

\footnotetext{
${ }^{20} \mathrm{~A}$ chi-square analysis found no difference in the proportion of females across groups, $\chi^{2}(1, \mathrm{~N}=27)=0.07, p=$ 79 .

21 'Bland', 'black', 'odd' and 'tank' were removed from the original 59 used in experiment 2 as they potentially had negative connotations. 'Nun' was also removed because of its religious connotations.
} 
After 20 seconds the metronome beat was stopped and participants had to work together to chant in time or sequentially. The metronome beat used was the same in both conditions except for the tempo. In the synchrony condition the tempo was set at $60 \mathrm{bpm}$; for the sequential condition the tempo was set at $80 \mathrm{bpm}$. These tempos were selected after preexperimental trialling found these tempos were the easiest for participants to follow. The metronome beat was written on ProTools version 7.1. The rhythm was in a 3/4 time signature (three beats per repetition) with each beat assigned a different tone. The first beat consisted of a high pitched percussion instrument; the second beat consisted of a mid-pitched percussion instrument; and the third beat consisted of a low pitched percussion instrument. For the synchrony condition, participants were told to read words from the left hand column on the first beat, the middle column on the second beat, and the right hand column on the third beat. For the sequential condition the participant in the left-most chair was told to read words from the left hand column on the first beat, the participant in the middle chair from the middle column on the second beat, and the participant in the right-most chair from the right hand column on the third beat. Three tambourine beats were played before the metronome beat began.

In the synchrony condition participants were instructed that the goal of the activity was to speak the words in time with each other - meaning that they needed to consistently say the same words as each other at the same time. For the sequential condition participants were told that the goal of the activity was to speak the words out of time with each other - meaning that the leftmost participant would speak the leftmost words on the sheet, the middle participant the middle words, and the rightmost participants the rightmost word. To help motivate participants to follow instructions and to give them a sense of shared fate, participants were also told that the experimenter would be measuring how accurately they spoke in time/ out of time with each other through the use of a voice recorder.

\section{Stag hunt game}

Because the stag hunt game used a dichotomous variable, it was essential to find the appropriate pay off schedule. If the risk dominant choice was too attractive then it was possible that everyone would choose the safe, but lower option. If the pay-off dominant choice was too attractive then it was possible that everyone would choose the higher risk, but higher payoff option. This would lead to either a floor effect or to a ceiling effect. Ideally, both options needed to be attractive, with the safe option narrowly edging out the risky option 
so I could test whether the synchrony manipulation would be enough to tip the balance in favour of risky cooperation.

To find the appropriate payoff schedule I ran a small on-line survey keeping the norisk option payoff constant and varying the payoff of the risky option (labeled $x$ and $y$ respectively to prevent any label influencing participants' decision). Participants were asked to imagine they were in a group of three with two other strangers. Would they prefer option $x$ - a guaranteed payment of $\$ 5$; or option $y$ a conditional payment - if they choose $y$ and both of the strangers choose $y$, then they will get $\$ \mathrm{P}$, but if they choose $y$ and one or more of the strangers choose $x$, then they will get $\$ 0$. \$P varied over 10 questions from $\$ 6$ to $\$ 15$ in $\$ 1$ intervals. The order that $\$ \mathrm{P}$ was presented in was randomised for each participant. A total of 19 participants answered the survey. The average pay-off that participants switched from the risk dominant equilibrium to the pay-off dominant equilibrium was a $\$ \mathrm{P}$ value of $\$ 10$. At this payoff, 12 of the 19 participants (63\%) had chosen the no-risk option.

Of course, how participants think they might respond may not correspond to how participants actually behave when there is real money on the line. I piloted this pay-off matrix with real money on 21 participants in groups of 3. Only 4 participants (19\%) chose the norisk option, indicating a potential ceiling effect. To make the no-risk option more attractive, I increased the pay-off of the fixed reward option to $\$ 7$, and accentuated the risk of the conditional reward option by writing the risk of getting $\$ 0$ first before the potential pay-off of $\$ 10$. The final choice participants were given can be seen in Table 1 below.

Table 1. Participants' choices for the stag hunt game.

\section{Option X}

Option Y

\begin{tabular}{ll}
\hline A guaranteed payment of \$7, no matter & $\bullet \quad \$ 0$ if any of the other participants \\
what the other participants decide & choose X, BUT
\end{tabular}

- $\$ \mathbf{1 0}$ if all 3 participants choose $\mathrm{Y}$

You will not know what the other participants will choose

As with the public goods game used in experiment 3, participants were asked to sit in chairs facing separate walls to minimise any communication. Participants were also told directly not to communicate with any of the other participants. Instructions were read out by 
the experimenter and also written on a sheet handed to participants so that the instructions were known to be common knowledge (Friedman \& Sunder, 1994). Participants were then handed a slip in a lettered envelope for which they could confidentially indicate if they would prefer option X or Y. Envelopes were then collected and taken to an assistant in another room to calculate each player's earnings. Earnings were placed back in the same lettered envelope for participants to collect at the end of the experimental session. This ensured that participants' contributions remained confidential.

\section{Post-activity questionnaire}

Four items were created to measure perceived synchrony, two positive items: "how much did you feel you were coordinated with the other participants?", "how much did you feel the other participants and yourself spoke in unison with each other?"; and two negative items that were reverse coded: "how much did you feel you were disjointed with the other participants?", "how much did you feel the other participants and yourself spoke out-of-time with each other?" (Cronbach alpha $=.72)$. Participants were asked to rate their response on a 7-point Likert scale from 1 (Not at all) to 7 (Very much so). Participants were also asked to rate how difficult they found the speaking activity on the same 7-point scale.

One item was included to measure perceived cooperation on the same 7-point Likert scale: "how much did you feel you and the other participants cooperated during the task?".

Four items were created to measure the amount of attention paid to the other participants, two positive items: "how much did you pay attention to the other participants?", "how much did you find the other participants aided in doing the activity?"; and two negative items that were reverse coded: "how much did you try to ignore the other participants?", "how much did you find the other participants distracting?" (alpha=.72).

Similarity was measured with two items on the same 7 point Likert scale: "how much did you feel similar to the other participants?" and "how much did you feel different to the other participants" (reverse coded), (Cronbach alpha $=.76)$.

Four items were used to measure entitativity adapted from Lakens and Stel (2011). There were three positive items: "How much did you experience a feeling of togetherness with the other participants?", "How much did you feel you were on the same team with the other participants?", "How much did you feel you and the other participants were a unit?"; and one reverse coded item: "How much did you feel disconnected from the other participants?" (Cronbach alpha $=.79)$. 
To measure interdependent self-construal the IOS (Aron et al., 1992) was used again, but modified further. Examination of the distribution in the previous experiments revealed a positive skew with most participants responding in the lower half of the scale. Following Schubert and Otten's (2002) modified IOS scale, I extended the beginning of the scale with two further diagrams where the circles were separated at different distances. The self circle was reduced in size to be smaller than the group circle, and a horizontal line included. The amount of circle that overlapped followed the scale developed by Swann, Gomez, Seyle, Morales, and Huici (2009) and included the self being totally engulfed in the group in the final diagram (see Figure 13 below).

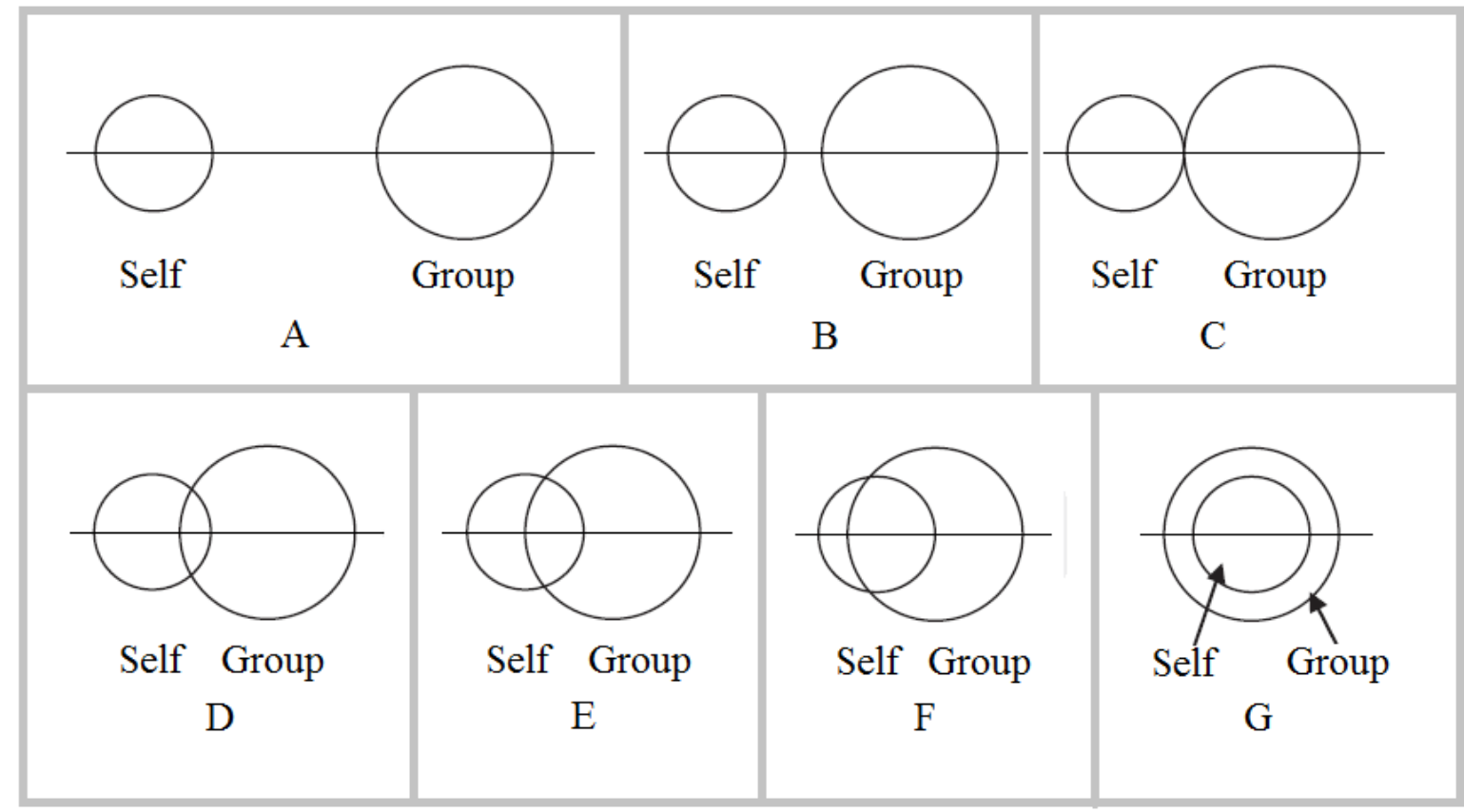

Figure 13. Modified Inclusion of Other in the Self scale (IOS).

Two trust questions about the economic game were used: "how much did you trust the other participants going into the payment choice game?" from 1 (did not trust at all) to 7 (trusted very much), and "how confident were you that the other group members would choose option Y (the $\$ 10$ or $\$ 0$ option )?” from 1 (not confident at all) to 7 (very confident) $($ Cronbach alpha $=.67)$.

As the 24-item University of Wales Institute of Science and Technology Mood Adjective Checklist (UMACL; Matthews, Jones, \& Chamberlain, 1990) was quite long and not of primary interest to the current study, the scale was reduced to 6 items - a positive and negative item for each of the 3 subscales. The items: happy, sad (hedonic tone; Cronbach alpha $=.32$ ), tense, relaxed (tense arousal; Cronbach alpha $=.67$ ), and energetic, tired 
(energetic arousal; Cronbach alpha =.12), were chosen for having high factor loadings for their relevant subscale on Matthews, Jones, and Chamberlains (1990) original factor analysis and having high face validity for what the subscales are meant to be measuring. The scale was also extend to a 7-point Likert scale to allow for more variance, anchored by not at all (1), moderately (4), and very much (7). Due to the low reliability for the hedonic tone and energetic arousal sub-scales, the individual items were tested separately.

As with the previous experiments, participants were also asked how well they knew each other before the study, their age, sex, and two open ended question asking participants to write any general comments they would like to make about the group activity they just did and one asking for comments about the survey or the study in general.

\section{Results}

\section{Pre-experiment bonding}

Across all groups, $89 \%$ of participants had never seen any of the other participants in their group before. Independent sample t-tests found no significant differences between the conditions in how well participants knew each other beforehand - using either the mean rating for each participant, $t(25)=1.73, p=.10$, or the highest rating for each participant, $t(25)=1.60, p=.12$.

\section{Manipulation check}

The manipulation produced a significant difference in perceived synchrony with participants in the synchrony condition $(M=5.50, S D=0.51)$ rating their condition as significantly more synchronous than participants in the sequential condition $(M=4.48, S D=$ $1.23), t(17.61)=2.84, p=.01, d=1.08 .^{22}$ The sequential condition $(M=3.29, S D=1.44)$ was rated as significantly more difficult than the synchrony condition $(M=2.08, S D=1.32)$, $t(25)=2.27, p=.03, d=.88$. However, both means were still on the lower half of the scale. This suggests that participants did not find either condition too difficult.

\footnotetext{
${ }^{22}$ In this thesis, when the degrees of freedom of a $t$-test in is not a whole number this indicates that Levene's Test for equality of variance was significant so Welsh's t-test (which doesn't assume equal variance) was used.
} 
A Pearson chi square found a significant association between the synchrony manipulation and the degree of cooperation, $\chi^{2}(1,27)=4.49, p=.03$, with $62 \%$ of participants in the synchrony condition choosing the cooperative $\mathrm{Y}$ option compared to $21 \%$ in the sequential condition (see Figure 14 below). Odds ratios indicated that the odds of a participant cooperating after moving in synchrony were 5.87 times higher than after moving sequentially.

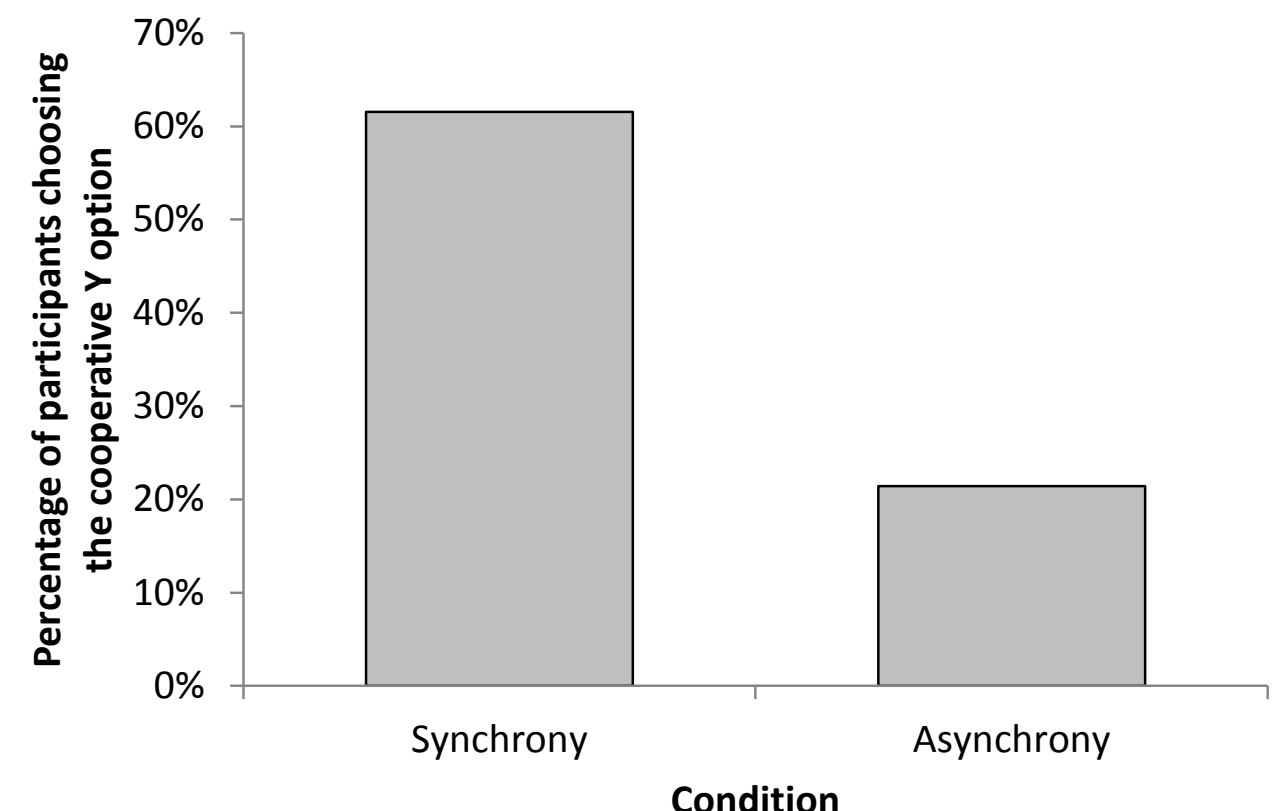

Figure 14. Percentage of participants choosing the cooperative $\mathrm{Y}$ option for each of the two vocalising conditions.

\section{Post-activity questionnaire}

There was no significant difference in perceived cooperation, $t(25)=0.07, p=.95$, attention, $t(25)=0.62, p=.54$, similarity, $t(25)=1.15, p=.26$, entitativity, $t(25)=0.87, p=$ .39 , self-construal, $t(25)=0.75, p=.26$, and trust, $t(25)=0.33, p=.75$. Participants in the sequential condition $(M=5.17, S D=1.19)$ were significantly happier after the activity than participants in the synchrony condition $(M=4.15, S D=1.07), t(23)=2.24, p=.04, d=.90$. However, happiness was not significantly correlated with the degree of cooperation $(r=-.07$, $p=.74)$ nor with any of the other self-report variables $\left(r_{\max }=.29, p=.17\right)$, except a 
marginally non-significant correlation with perceived similarity $\left(r_{\max }=.37, p=.07\right)$. The only self-report variable that was correlated with cooperative behavior was trust $(r=.75, p<.01)$.

\section{Discussion}

The aim of experiment 4 was to evaluate whether phase-locked synchronous chanting created with shared intentionality would produce a significantly greater level of cooperation than non-phase-locked chanting created with shared intentionality. Analysis revealed that participants who chanted in phase-locked synchrony were more willing to cooperate than participants who chanted sequentially. If we compare this result to experiment 2 in which no difference between the synchrony and asynchrony conditions were found, the difference between the vocalising conditions in experiment 4 suggests that shared intentionality is an important component in producing a synchrony-cooperation effect for activities involving vocalisation.

The lack of any significant differences in the self-report measures (except for the manipulation check of perceived synchrony) was in contrast to the finding of experiment $3 \mathrm{~b}$ where analysis revealed large differences in interdependent self-construal between the synchrony with shared goal condition and the original asynchrony condition. This discrepancy could suggest that it was the use of shared intentionality rather than an interaction between synchrony and shared intentionality that produced the large effects seen in the self-report data from experiment $3 \mathrm{~b}$. Another possibility for why no difference in the self-report measures was found was that chanting sequentially, although perceived as not being as in-sync as the synchrony condition, is still perceived as being more coordinated than the asynchrony conditions previously used. In the sequential conditions, although participants are not chanting at the same time they are still entrained to a common underlying beat. If it was possible for participants to chant at different speeds so that there was no entrainment at all (as in the original asynchrony condition used in experiment 2) differences in self report measures may have occurred.

To better assess the interaction of synchrony and shared intentionality on cooperative behaviour and self-report measures, it was important to manipulate both synchrony and shared intentionality. This was the objective of experiment 5. Experiment 5 also included an asynchrony condition as well as a sequential condition to examine the relative importance of phase-relationship in producing a synchrony-cooperation effect. Given the difficulties with 
manipulating synchronous chanting, I returned to body movement manipulations of synchrony.

\section{Experiment 5}

\section{Overview}

Experiment 5 sought answers to three questions: (1) do synchrony and shared intentionality interact; (2) what psychological processes underlie the synchrony-cooperation effect; and (3) does frequency-locked synchrony as well as phase-locked synchrony produce an increase in cooperation? To help answer the first question, synchrony was manipulated with either a shared goal or an individual goal. Based on the mechanisms discussed in the chapter introduction (see page 70), I hypothesised that a significant interaction between synchrony and shared intentionality would be found. Specifically, I predicted that the difference in cooperation between the synchrony and control conditions should be greater when shared intentionality is used to create synchrony than when only an individual goal is used to create incidental synchrony.

Concerning the second question, as with experiment 4, similarity, interdependent selfconstrual, entitativity, trust, perceived synchrony, perceived cooperation and attention directed towards the other participants were measured to examine their role in mediating any effect. Path analysis was used to assess the two theoretical mechanisms discussed in the chapter introduction. The joint attention model (Figure 15 on page 91) suggests that having a shared goal increases the attention paid towards the other participants, which in turn increases perceived synchrony in the synchrony condition. Greater perceived synchrony leads to greater perceived similarity and self-construal (through shared perception-action circuits) and greater perceived entitativity (through gestalt grouping mechanisms) ${ }^{23}$. This in turn increases trust $^{24}$ and cooperative behaviour. The reinforcement of cooperation model (Figure 16 on page 91) suggests that having a shared goal increases perceived cooperation if participants obtain feedback that the group has been successful in achieving that goal. If the goal is to create synchrony, success is gathered from whether or not they perceive synchrony.

\footnotetext{
${ }^{23}$ The relationship between entitativity, similarity and self-construal is not modelled due to the potential bidirectional relationship between all three constructs (see page 37 ).

${ }^{24}$ Trust specifically is used in these models because it is reasoned that it is key for cooperating in the stag hunt game (see page 78).
} 
Perceived cooperation could lead to greater trust mediated by entitativity, similarity and interdependent self-construal, and so in turn greater cooperation.

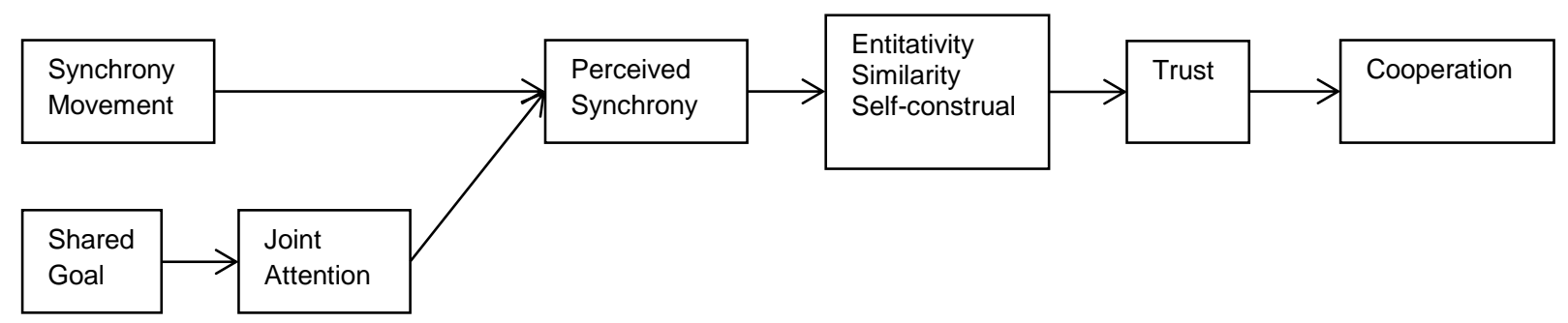

Figure 15. The joint attention model

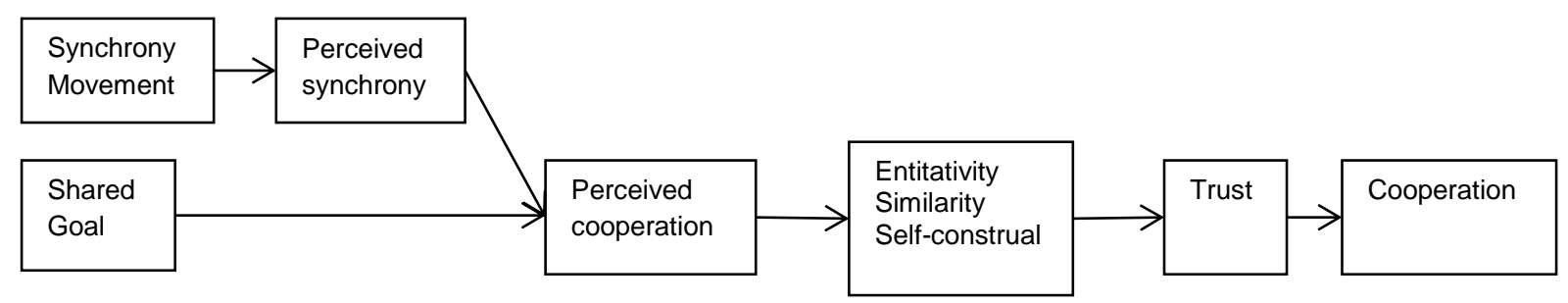

Figure 16. The reinforcement of cooperation model

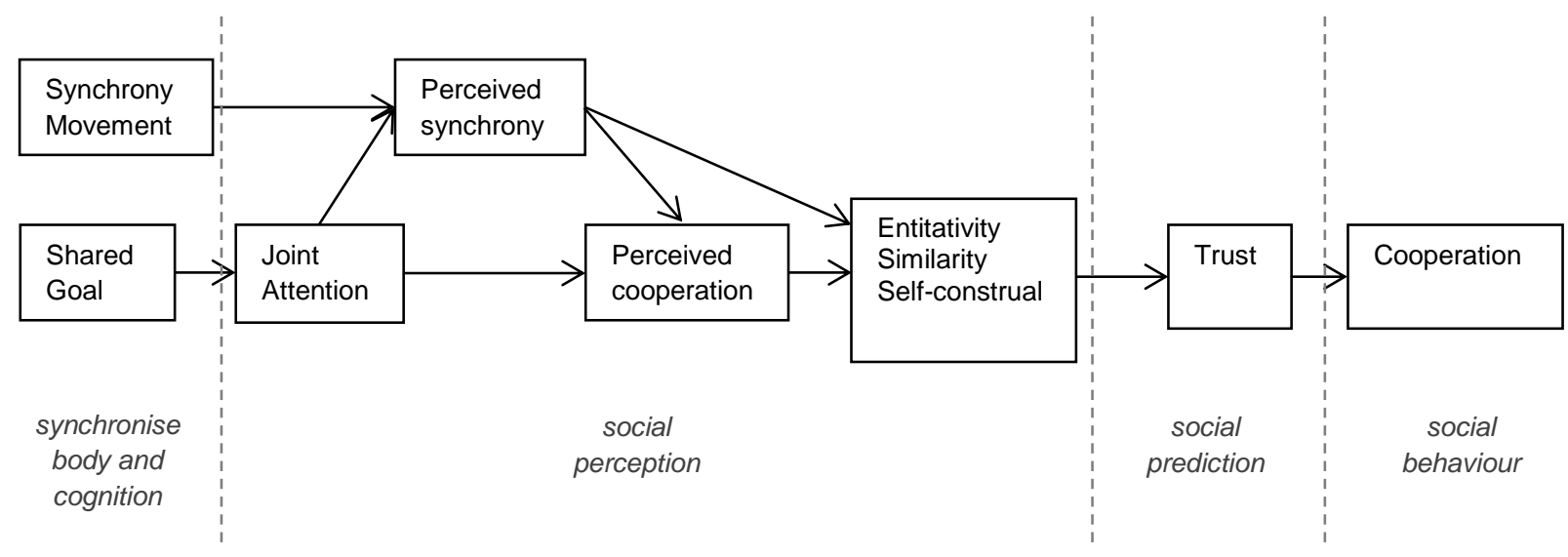

Figure 17. The combined joint attention and reinforcement of cooperation model

Note that these models are not mutually exclusive: it is possible that both could be operating. A combined model (Figure 17 above) was tested for the path analysis. This combined model suggests that the perceived synchrony used as evidence for perceived cooperation could be further strengthened by the increased joint attention produced by the shared goal; i.e., when a group shares the goal of moving in synchrony, the increased joint attention needed to create synchrony also results in increased feedback that they are 
successful at their goal. The models could therefore reinforce each other. The combined model also includes a direct pathway from perceived synchrony to entitativity, interdependent self-construal and similarity (as per the joint attention model), as well as an indirect one mediated through perceived cooperation (as per the reinforced cooperation model). To put simply, the model suggests that synchronising both body and cognition influences the performer's social cognition, which influences their social predictions, which influences their social behaviour.

To help answer the third question, two control conditions were used for comparison to the synchrony condition: a condition where participants were entrained to a common beat but moved out of phase with each other (sequential); and a condition where participants moved at different speeds so were neither phase-locked nor frequency-locked to each other's movements (asynchrony). The significant difference in cooperation between the synchrony and sequential conditions found in experiment 4 suggest that being in-phase does increase cooperation compared to being out of phase but entrained. It was therefore predicted that the synchrony condition would be significantly greater than both the sequential and asynchrony condition. If being phase-locked is the critical factor for producing a synchrony-cooperation effect then it would be predicted that there should be no difference in the level of cooperation between the sequential and the asynchrony condition. If being frequency entrained has an effect - even out of phase - then there should also be a difference between the sequential and asynchrony conditions.

Because of the difficulty in asking participants to chant out of time with each other, silent movement was used again. Unlike with the chanting in experiment 2 , in experiment 3 participants did not rate moving out of time significantly more difficult than the synchrony condition. However, the question remains whether intentionally trying to move out of synchrony is possible. A number of studies have shown that people often automatically become entrained to other people's rhythms if they are performing similar actions at a similar speed (e.g., Zivotofsky \& Hausdorff, 2007; see page 9). Even though participants are consciously trying to move out of time they may still end up falling in time with each other (see Kirschner \& Tomasello, 2010). For example, Issartel, Marin, and Cadopi (2007) showed that even when participants are consciously trying not to coordinate they are still affected by each other's movements. The authors found that the movement of two participants who were explicitly told not to coordinate was more coordinated than each participant acting on their own. This suggests that information and adaptation of movement to each other was occurring between participants. However, the condition in which participants were explicitly trying not 
to coordinate was still much less coordinated than the condition in which participants were trying to coordinate i.e., participants could still move out of time even though each other's movements had some influence on the other. This could suggest that although intentionally moving out of synchrony will be less synchronous than the synchrony condition, it will be more coordinated than an asynchrony condition in which participants are able to entrain to a metronome beat. Furthermore, in Issartel et al.'s study participants were not required to move rhythmically. Intentionally trying to move rhythmically out of time may be easier as one could concentrate on their own rhythm; or it could be harder because it is more difficult to ignore rhythmic information. I ran a few trial groups to see if participants were able to intentionally move out of time. I found that it was possible if participants knew how to adjust the speed of their movements if they found themselves falling in time: if one of the participants was instructed to speed up if they found themselves in time and another asked to slow down. However, the degree of asynchrony was just assessed visually. A more precise method was needed to measure the timing of participants' behavior. To do this, foot-pedals were introduced that relayed information about the timing of participants' movements to a computer.

\section{Method}

\section{Participants}

Participants were 86 volunteers recruited through the same method as described for experiment 4 (61 female; mean age $=23.71$, range: $16-60$ years). All groups included three participants. The distribution of females was evenly spread across conditions. ${ }^{25}$

\section{Procedure}

I investigated the interaction of shared goals and synchrony using a 3 (movement) $x 2$ (goal) factorial design. The three levels of the movement manipulation were: (1) synchrony participants moved the same way at the same time; (2) sequential - participants moved at different times but still entrained to the same beat; and (3) asynchrony - participants moved at different speeds resulting in them moving out of time with each other. The two levels of

\footnotetext{
${ }^{25}$ A log-linear analysis with movement, membership and sex found no significant interactions, only a main effect of sex (there were more females than males in the sample).
} 
the goal manipulation were: (1) shared goal - participants worked together to complete a shared goal (that is they used shared intentionality); and (2) individual goal - participants were told to move in time with the metronome beat coming through their headphones. (See Table 2 for a summary of the conditions).

Table 2. Summary of the six conditions and the goals participants were given

$$
\text { Movement condition }
$$

\begin{tabular}{llll}
\cline { 2 - 4 } Goal condition & Synchrony (Sy) & Sequential (Sq) & Asynchrony (As) \\
\hline Group Goal (GG) & Work together to & Work together to & Work together to \\
& move in time & move sequentially & move out of time \\
& (SyGG) & (SqGG) & (AsGG) \\
Individual Goal (IG) & $\begin{array}{l}\text { Move in time with } \\
\text { beat (SyIG) }\end{array}$ & Move in time with & Move in time with \\
& & & beat (SqIG) \\
\hline
\end{tabular}

\section{Synchrony manipulation}

The individual goal condition was similar to experiment 1 and experiment 3 where participants heard the metronome beat for the whole length of the movement activity. Instead of the three different movements, participants were asked to step for the full six minutes. This allowed for the use of foot-pedals to measure the relative timing of participants' movements. In the synchrony condition (SyIG) participants were played the same metronome beat (55bpm) using the headphone splitter connected to the same digital audio player. The metronome beat was the same stimulus used in experiment 1 and $3-$ a rhythm in $4 / 4$ time with the first beat accented. For the asynchrony condition (AsIG) each participant was played a metronome beat at a different speed $(45 \mathrm{bpm}, 55 \mathrm{bpm}, 65 \mathrm{bpm})$ from different digital audio players. The tempos used were slightly slower than in studies one and three because a small trial found these tempos were easiest for participants to do the stepping motion. Four tambourine beats were played before the metronome beat began to prepare participants for the start of the beat. Participants were told to move their left foot on the first accented beat, the left foot back on the second beat, the right foot forward on the third beat, and the right foot back on the fourth beat. Participants were also asked to sway their arms. To make the 
movements seem less like a normal walking motion and to make it easy to coordinate with other participants, participants moved their left arm forward with their left leg, and right arm forward with their right leg. The experimenter explicitly told participants that the goal was to move in time with the rhythmic pulse played through their headphones.

For the sequential condition (SqIG) a different metronome beat had to be created to aid in coordinating participants. As there were 3 participants each moving in turn, the beat was in $6 / 8$ time ( 6 beats per repetitive unit) at $45 \mathrm{bpm}$. The first two beats were a low pitched drum, the next two beats were a mid-tone drum, and the last two beats were a high pitched drum. The left most participant moved their left foot forward (along with their left arm) and back on the first 2 beats, middle participant moved their left foot forward and back on the next 2 beats, then right-most participant moved their left foot forward and back on the last two beats. This procedure was repeated with the right foot, then again with the left, and so on.

For the synchrony-group goal condition (SyGG) participants were instructed that the goal of the movement was to move in time with each other meaning that that they needed to consistently press the foot-pedals at the same time as each other, and move at the same speed. For the sequential-group goal condition (SqGG) participants were instructed that the goal of the movement was to move sequentially and at the same speed as each other. For the asynchrony-group goal condition (AsGG) participants were instructed that the goal of the movement was to move out of time with each other meaning that that they should not be consistently pressing the foot-pedals at the same time as each other, but should move at different speeds. Participants in the AsGG condition were also told that occasionally they may end up pressing the pedals at the same time as another - this possibility is inevitable, but as long as simultaneous foot-pedal presses were not consistently the case. To help participants initially coordinate, the appropriate metronome beat for the condition was played for the first 20 seconds of their movement. Participants were told that after 20 seconds the pulse will stop and they will need to work together to stay in time/ move sequentially/ stay out of time. To help keep participants out of time in the asynchrony condition, the left-most participants who initially heard the slowest $45 \mathrm{bpm}$ metronome was told to slow the speed of their movement down a little if they found themselves moving in time, the rightmost participants who initially heard the fastest $65 \mathrm{bpm}$ metronome was told to speed up a little if they found themselves moving in time. This procedure was developed from pre-experimental trials as an effective method for getting participants to move out of time with each other.

The salience of the goals was accentuated by having foot-pedals $(250 \mathrm{~V}, 10 \mathrm{amp}$, from www.jaycar.co.nz cat. no.: SP0760; see Figure 18 on page 96) and a video camera and telling 
participants that their own performance (individual goal condition) or the group performance (group goal condition) was being measured. Participants in the individual goal conditions were also told that "it is important that you do the best you can to keep in time with the pulse" and in the group goal conditions that "it is important that you work together to keep in time/ keep coordinated/ keep out of time with each other."

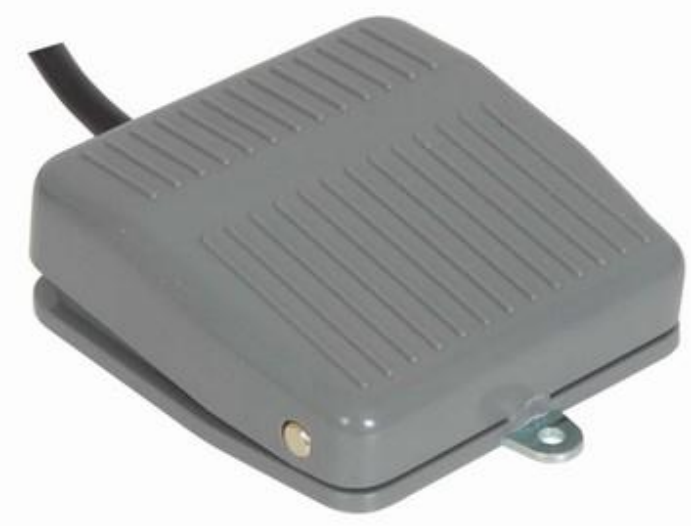

Figure 18. Photograph of the foot-pedal used in experiment 5. Note. From www.jaycar.co.nz.

\section{Foot-pedal timings}

Analysis of foot-pedal data was used as a manipulation check. A program was written in C programming language and run on MS-DOS to record timings of the foot-pedals through a parallel port on to a Pentium D, Microsoft Windows XP, Dell Optiplex 745. This left a time stamp for the appropriate foot-pedal. The resolution was to $10 \mathrm{~ms}$. The time stamp was in absolute time (hour-minute-second-millisecond), so was converted into relative timing by converting to milliseconds then subtracting each time stamp from the very first pedal press of the experimental session. To measure synchrony within a group, the mean phase difference and the variance of phase differences was calculated using circular statistics (Fisher, 1993; see Appendix 3 on page 142 for details on the statistical analyses of these data). All participants in the synchrony condition should be in-phase with each other all of the time. This should result in the distribution of phase differences between any two participants in a group being centered on $0^{\circ}$ with little variance. All participants in the sequential condition should be $240^{\circ}$ out of phase with each other all of the time. This should result in the distribution of phase differences between any two participants in a group being centered on 
$240^{\circ}$ with little variance. All participants in the asynchrony condition should have no stable phase relationship with each other. This should result in the distribution of phase differences between any two participants in a group being evenly distributed across all phase relationships.

\section{Stag hunt game \& post-activity questionnaire}

Experiment 5 used the same stag-hunt protocols as per experiment 4 . The same postactivity questionnaire from experiment 4 was also used with measures for perceived synchrony $($ Cronbach alpha $=.83)$, similarity $($ Cronbach alpha $=.56)$, attention $($ Cronbach alpha $=.72)$, entitativity $($ Cronbach alpha $=.77)$, trust $($ Cronbach alpha $=.81)$, and mood (hedonic tone: Cronbach alpha $=.49$; tense arousal: Cronbach alpha $=.68$; energetic arousal: Cronbach alpha $=.51)$ and the one item measures of cooperation and interdependent selfconstrual.

\section{Results}

\section{Pre-experiment bonding}

Two participants were removed who were in the same group and were in a committed romantic relationship together. Two participants were also removed due to poor English (they spent nearly 3 times as long as other participants completing the questionnaire). Of the remaining 82 participants, $85 \%$ had never seen any of the other participants before. Two $3 \times 2$ ANOVAs with movement and goal as the independent variables and either the mean rating for each participant or the highest rating for each participant as the dependent variable found no significant main effects or interactions in how well participants knew each other beforehand, $F_{\max }=1.69, p=.19$.

\section{Manipulation check}

\section{Foot-pedal timings}

Analyses of the foot-pedal timings with circular statistics (see Appendix 3 on page 142) found, as expected, that the distribution of phase relationships for all participants in both SyGG and SyIG conditions were highly unimodal and centred on in-phase $\left(0^{\circ}\right)$ synchrony. Participants in the SyIG condition had slightly less variance in phase match than participants 
in the SyGG condition - that is, participants were slightly more synchronised in the SyIG condition. Importantly, comparison of the variance of phase match between the synchrony and asynchrony condition found that participants in the synchrony condition were significantly better entrained than in the asynchrony condition. In the asynchrony condition, most participants had highly distributed phase relationships suggesting limited entrainment. There were 5 participants in the AsGG condition ( 3 from one group, 2 from another group) that appeared to have a slight bias towards being anti-phase $\left(180^{\circ}\right)$ with each other. However, visual inspection of the distribution of phase angles for these participants was still highly distributed. All participants in the sequential condition had highly unimodal distributions centred on $240^{\circ}$ out of phase. There was no difference in in the variance of phase match between the SqGG and SqIG conditions indicating that participants in both conditions were equally good at entraining. There was also no difference in the variance of phase match between the synchrony and sequential conditions, but participants in the sequential condition were significantly better entrained than in the asynchrony condition. Overall, although the SyGG was not as synchronous as the SyIG condition and AsGG was not as asynchronous as the AsIG condition, these differences were minor compared to the differences between movement conditions. Therefore, the movement manipulation worked.

\section{Perceived synchrony}

The movement manipulation was also tested with a 3 (movement) x2 (goal) factorial ANOVA on perceived synchrony. As expected there was a main effect of synchrony, $F(2,76)$ $=42.86, p<.01, \eta_{\mathrm{p}}{ }^{2}=.53$, but no main effect of goal, $F(1,76)=0.83, p=.37, \eta_{\mathrm{p}}{ }^{2}=.01$. However, there was a significant Movement $x$ Goal interaction, $F(2,76)=4.59, p=.01, \eta_{\mathrm{p}}{ }^{2}=$ .11 , (see Figure 19 on page 99). Exploration of this interaction using Gabriel post-hoc tests found that for the group goal condition, both the sequential $(M=4.02, S D=1.11)$ and asynchrony $(M=3.25, S D=1.03)$ conditions were significantly different from the synchrony condition $(M=5.27, S D=0.90 ; p=.01, d=1.24$ and $p<.01, d=2.09$ respectively). The sequential condition was not significantly different from the asynchrony condition $(p=.15, d$ $=0.72)$. For the individual goal condition, the asynchrony condition $(M=2.33, S D=0.90)$ was significantly different from the synchrony condition $(M=4.98, S D=0.65, p<.01, d=$ 3.38). The sequential condition $(M=4.65, S D=1.05)$ was not significantly different from the synchrony condition ( $p=.70, d=0.38$ ) but was significantly different from the asynchrony condition $(p<.01, d=2.37)$. Independent t-tests comparing group goal to individual goal for each of the three levels of the movement variable only found a significant difference between 
group goal and individual goal for the asynchrony condition, $t(25)=2.469, p=.02, d=0.95$, with the individual goal condition having a significantly lower perceived synchrony than the group goal condition. These data suggest that in terms of perceived synchrony the manipulation worked for the synchrony and asynchrony conditions. However, the sequential condition still appears to be perceived as reasonably synchronous in the individual goal condition. The lower level of perceived synchrony for AsIG compared to AsGG could be because participants were better at moving asynchronously when they had the beat to guide them. This matches the foot-pedal data.

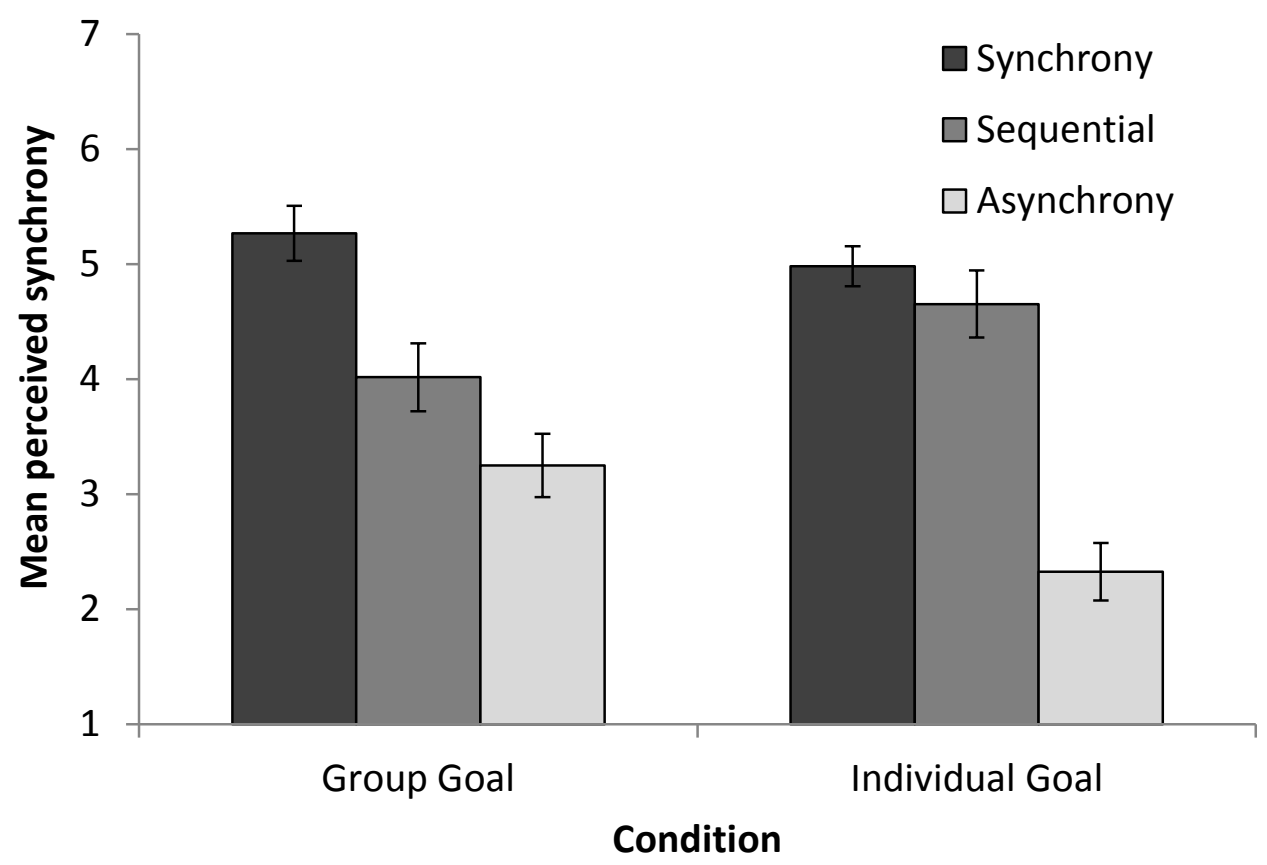

Figure 19. Mean perceived synchrony for when synchronous, sequential and asynchronous movement is created through a group goal or an individual goal. Error bars are +/- SE.

\section{Perceived cooperation}

Perceived cooperation could also serve as a manipulation check for the goal manipulation. Specifically, the group goal manipulation should have been perceived as more cooperative than the individual goal condition. Indeed, a 3 (movement) x2 (goal) factorial ANOVA on perceived cooperation revealed that there was a main effect of goal, $F(1,76)=$ $6.02, p=.02, \eta_{\mathrm{p}}{ }^{2}=.07$ with the group goal condition having a higher mean $(M=4.39, S D=$ 1.38) than individual goal condition $(M=3.61, S D=1.44 ; d=0.55$; see Figure 20 on page $100)$. Notably, there was also a main effect of synchrony, $F(2,76)=7.36, p<.01, p<.01, \eta_{\mathrm{p}}{ }^{2}$ $=.16$. Gabriel post-hoc tests revealed the synchrony condition $(M=4.54, S D=1.22)$ had a 
significantly higher mean than the asynchrony condition $(M=3.26, S D=1.61 ; p<.01, d=$ $0.90)$. The sequential $(M=4.22, S D=1.24)$ condition was also significantly higher than the asynchrony condition $(p=.03, d=0.67)$. There was no difference between the synchrony and sequential conditions $(p=.86, d=0.26)$. The Movement $\mathrm{x}$ Goal interaction was not significant, $F(2,76)=2.27, p=.27, \eta_{\mathrm{p}}^{2}=.03$.

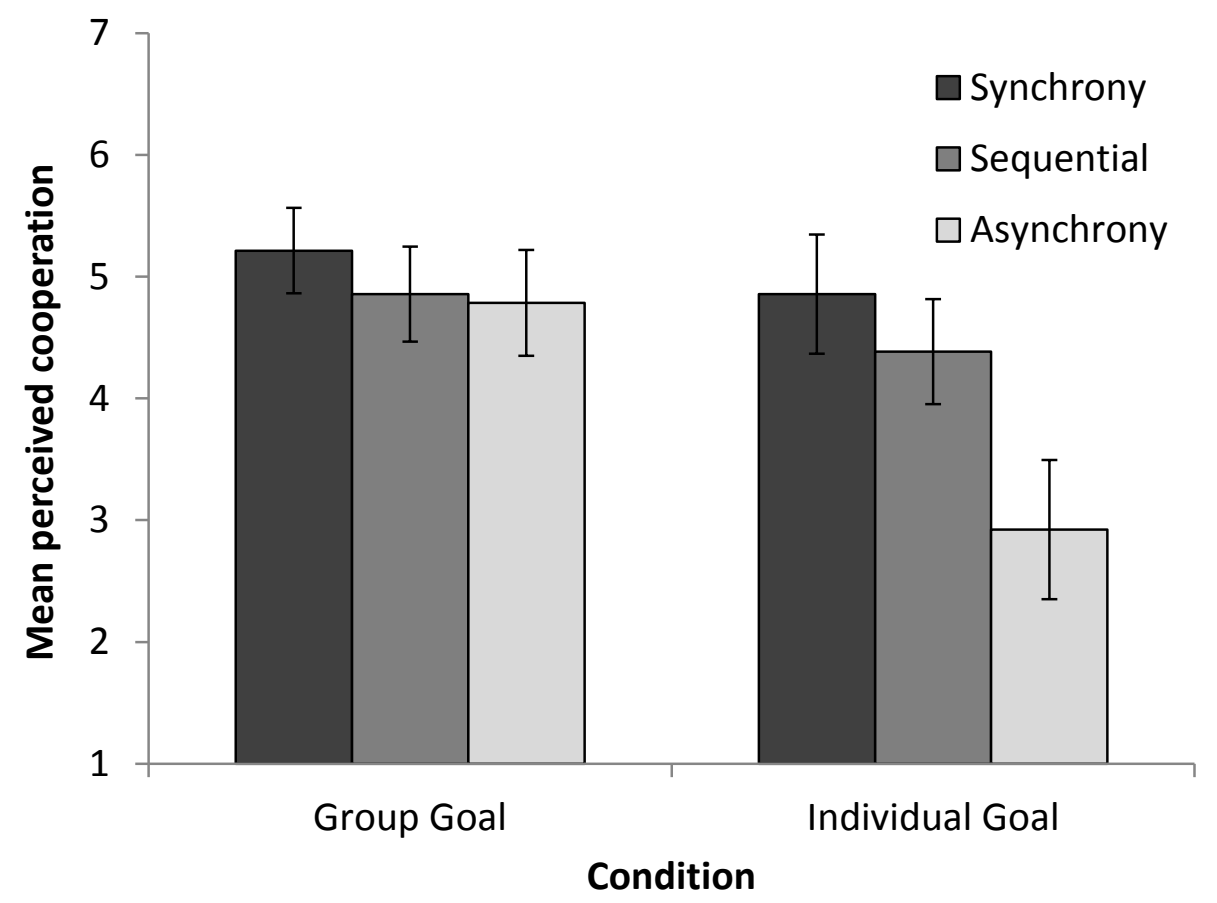

Figure 20. Mean perceived cooperation for when synchronous, sequential and asynchronous movement is created through a group goal or an individual goal. Error bars are +/- SE.

\section{Perceived difficulty}

In terms of perceived difficulty of the movements, there was only a significant Movement x Goal interaction, $F(2,76)=5.14, p=.01, \eta_{\mathrm{p}}{ }^{2}=.12$. Neither of the main effects were significant, $F_{\max }=1.08, p=.34$. Independent t-tests comparing group goal to individual goal for each of the three levels of the movement variable only found a significant difference between group goal and individual goal for the asynchrony condition, $t(16.53)=2.64, p=$ $.02, d=1.85$, with the group goal condition having a significantly higher perceived difficulty $(M=3.29, S D=0.90)$ than the individual goal condition $(M=1.77, S D=0.73)$.

\section{Cooperation measure}

To examine the effect of the manipulations on cooperation, a three-way hierarchical log-linear analysis with backward elimination was conducted with movement (3 levels), goal 
(2 levels), and cooperative behaviour (2 levels). A significant Movement x Goal x Behaviour interaction was found, $\chi^{2}(2,82)=7.94, p=.02$. Neither the Movement $\mathrm{x}$ Behaviour interaction, $\chi^{2}(2,82)=3.21, p=.20$, nor the Goal $\mathrm{x}$ Behaviour interaction, $\chi^{2}(1,82)=1.29, p=$ .26 , were significant. Follow-up chi-square tests to break down the significant three-way interaction found a significant difference in the group goal condition, $\chi^{2}(2,42)=8.68, p=$ .01 . The synchrony condition was significantly different from both the sequential condition, $\chi^{2}(1,28)=6.30, p=.01$, and the asynchrony condition, $\chi^{2}(1,28)=8.02, p=.01$. Odds ratios indicated that the odds of a participant cooperating after moving in synchrony was 13.00 times higher than after moving sequentially and 17.33 times higher than after asynchronous movement. No significant difference existed between the sequential and asynchrony condition, $\chi^{2}(1,28)=.14, p=.71$. No significant differences were found in the individual goal condition, $\chi^{2}(2,40)=1.06, p=.59$. For the individual goal condition odds ratios indicated that the odds of a participant cooperating after moving in synchrony was 2.13 times lower than after moving sequentially and 1.17 times lower than after asynchronous movement.

As these analyses and Figure 21 shows, the three-way interaction is primarily driven by the condition where participants worked together to create synchrony. In this condition $93 \%$ of participants choose the cooperative Y option compared to between $43 \%-62 \%$ in the other conditions.

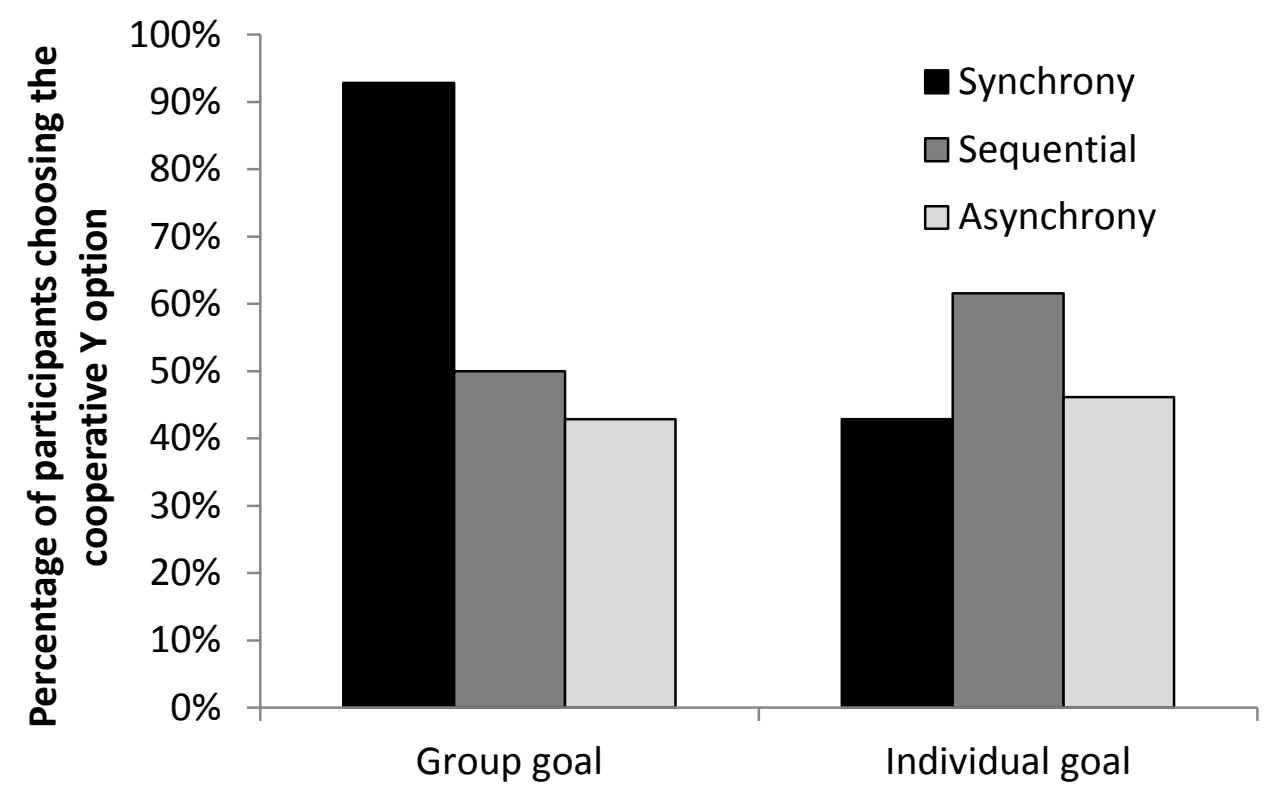

Condition

Figure 21. Percentage of participants choosing the cooperative $\mathrm{Y}$ option for when synchronous, sequential and asynchronous movement is created through a group goal or an individual goal. 
A 3 (synchrony) x2 (goal) factorial ANOVAs were used to test entitativity, similarity, interdependent self-construal, attention, trust and the mood measures. For entitativity, there was no significant interaction or main effect of goal $\left(F_{\max }=1.69, p=.19\right)$, only a significant main effect of movement was found, $F(2,76)=6.61, p<.01, \eta_{\mathrm{p}}{ }^{2}=0.15$. Gabriel post-hoc tests revealed that the synchrony condition $(M=4.66, S D=1.01)$ had a significantly higher mean than the asynchrony condition $(M=3.58, S D=1.26 ; p<.01, d=0.95)$ but not significantly higher than the sequential condition $(M=4.34, S D=1.14 ; p=.66, d=0.30$; see Figure 22 below). The sequential condition was significantly higher than the asynchrony condition $(p=.05, d=0.63)$. Entitativity was significantly correlated with participants' choice of the cooperative Y option $\left(r_{\mathrm{pb}}=.27, p=.01\right)$.

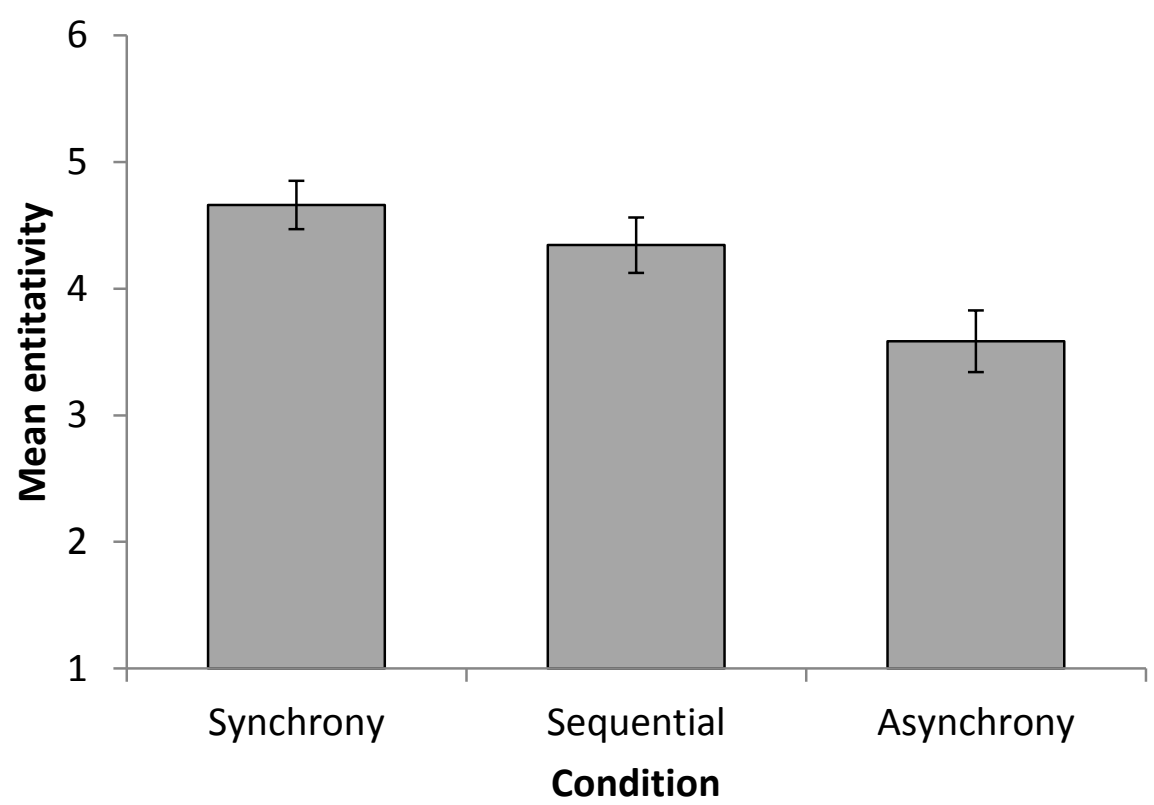

Figure 22. Mean entitativity for each of the movement conditions. Error bars are +/- SE.

A significant main effect of synchrony was also found for perceived similarity, $F(2,76)=4.25, p=.02, \eta_{\mathrm{p}}{ }^{2}=0.10$. Gabriel post-hoc tests only found a significant difference between the synchrony $(M=4.71, S D=1.18)$ and asynchrony conditions $(M=3.72, S D=$ $1.35, p=.01, d=0.83$; see Figure 23 on page 103 ). The difference between the synchrony and sequential conditions $(M=4.20, S D=1.20)$ was not significant, $p=.35, d=0.43$, nor was the difference between the sequential and asynchrony conditions, $p=.40, d=38$. No 
significant interaction or main effect of goal was found, $F_{\max }=0.21, p=.82$. Perceived similarity was significantly correlated with participants choice of the cooperative Y option $\left(r_{\mathrm{pb}}=.27, p=.02\right)$.

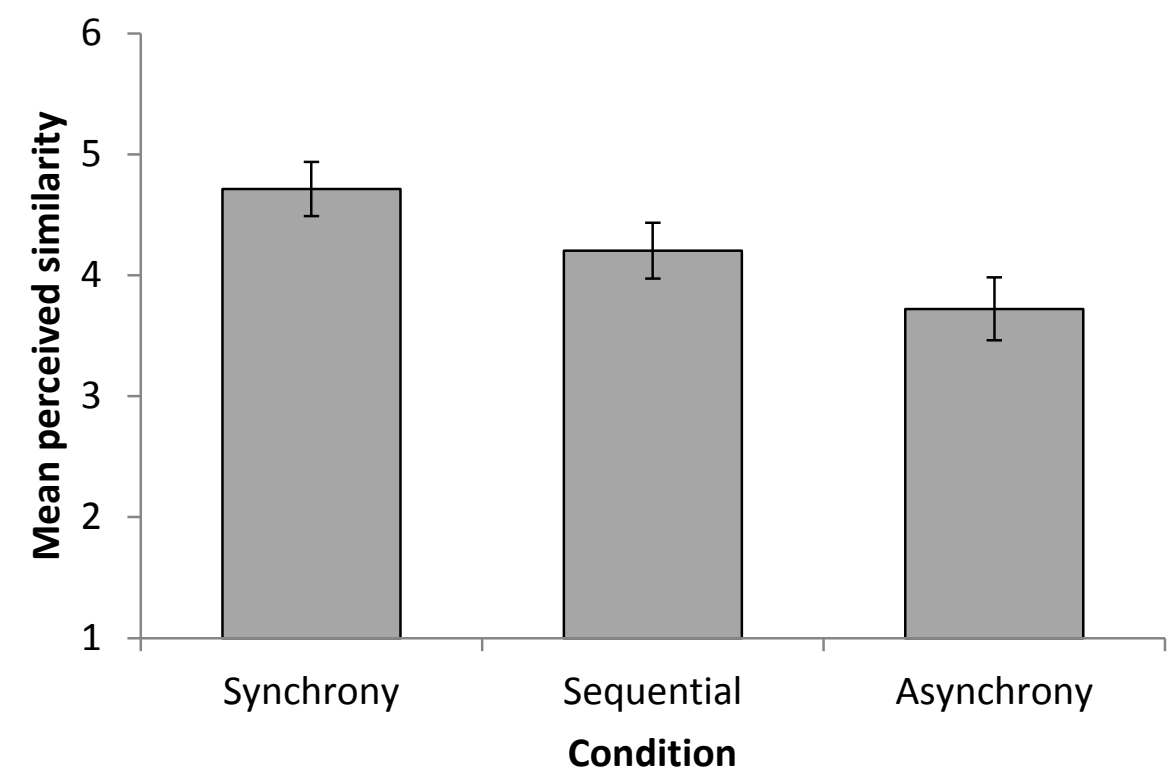

Figure 23. Mean perceived similarity for each of the movement conditions. Error bars are +/SE.

For attention directed towards other participants (see Figure 24 on page 104, there was both a significant main effect of synchrony, $F(2,76)=6.46, p<.01, \eta_{\mathrm{p}}{ }^{2}=0.15$, and a significant main effect of goal, $F(2,76)=6.15, p=.02, \eta_{\mathrm{p}}{ }^{2}=0.08$. There was no significant interaction, $F(2,76)=1.80, p=.17, \eta_{\mathrm{p}}{ }^{2}=0.05$. Exploring the main effect of synchrony, Gabriel post-hoc tests found that participants paid more attention to their fellow participants in the synchrony condition $(M=5.26, S D=1.04)$ than the asynchrony condition $(M=4.18$, $S D=1.46 ; p<.01, d=85)$. The sequential condition $(M=4.98, S D=1.17)$ was also significantly higher than the asynchrony condition $(p=.04, d=0.60)$, but was not significantly lower than the synchrony condition $(p=.76, d=0.25)$. For the main effect of goal, the group goal condition $(M=5.12, S D=1.27)$ had a significantly higher mean than the individual goal condition $(M=4.49, S D=1.27 ; d=0.50)$.

No main effects or interactions were found for trust, $F_{\max }=1.00, p=.37$ nor selfconstrual $F_{\max }=0.85, p=.43$. No main effects were found for hedonic tone, tense arousal, and energetic arousal, $F_{\max }=0.78, p=.46$. Because of the low internal consistency reliability for hedonic tone and energetic arousal $3 \times 2$ factorial ANOVAs were also 
conducted on the individual items. Again no significant main effects or interactions were found, $F_{\max }=2.68, p=.08$.

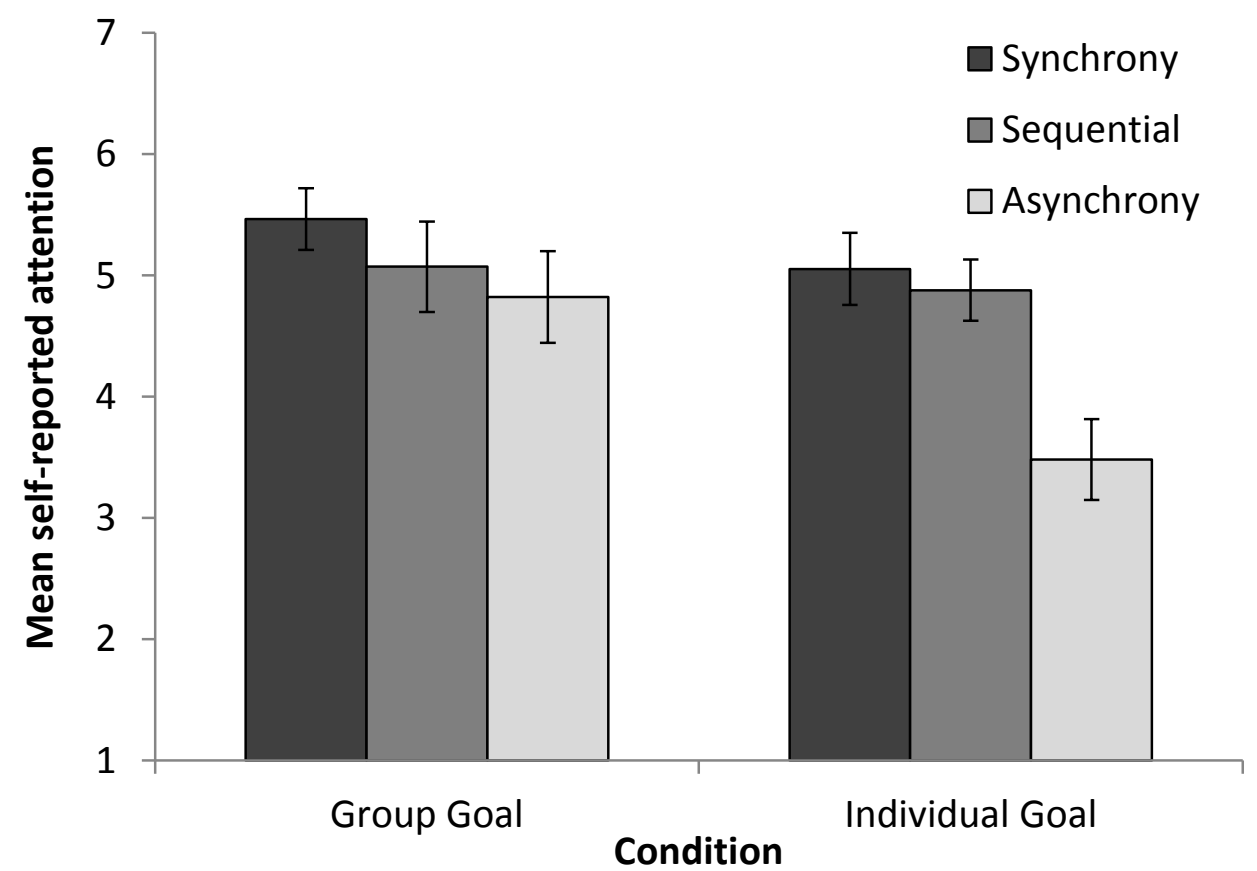

Figure 24. Mean attention directed towards the other participants for when synchronous, sequential and asynchronous movement is created through a group goal or an individual goal. Error bars are +/- SE.

Path Analysis

The combined model (see Figure 17 on page 91) was tested using MPlus (Muthén \& Muthén, 2010). As similarity and interdependent self-construal were both strongly positively correlated with entitativity $(r=.63, p<.01$ for similarity and entitativity; $r=.59, p<.01$ for interdependent self-construal and entitativity) and all three measures could potentially be related to each other bi-directionally (see page 39), a combined measure including the items from all three scales was created. A principal components factor analysis on the seven items (4 entitativity items, 2 similarity items, 1 self-construal item) with varimax rotation confirmed that there was only one factor using Kaiser's criterion of eigenvalues greater than 1. This factor (termed social cohesion) explained $52.86 \%$ of the variance. The internal consistency reliability of this social cohesion measure was also good (Cronbach alpha $=.82)$. Because the synchrony manipulation had three levels, two dummy codes were used. Because my main interest was the effect of synchrony compared to the sequential and asynchrony 
conditions one dummy variable compared the synchrony condition to the asynchrony condition and the other compared the synchrony condition to the sequential condition. The combined model provided a very good fit: $\chi^{2}(23)=28.90, \mathrm{p}=.18$; CFI $=.96$, RMSEA $=.06$, $\mathrm{WRMR}=.67^{26}$ (see Figure 25 below for standardised coefficients).

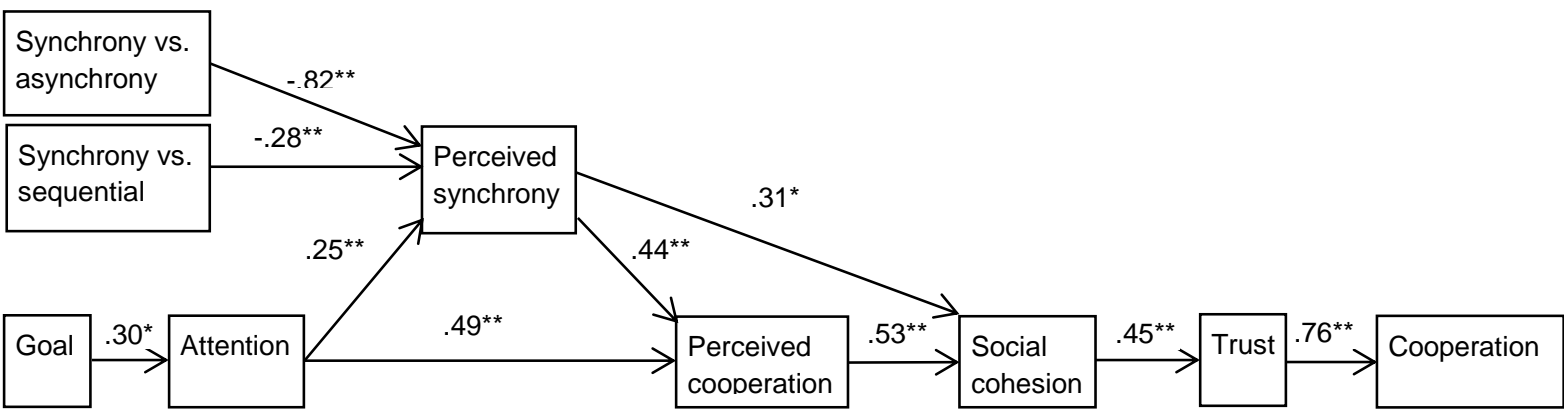

Figure 25. Standardised coefficients for the combined joint attention and reinforcement of cooperation model $(*<.05 ; * *<.01)$.

\section{Discussion}

In terms of cooperative behaviour, the results from experiment 5 were quite striking. When participants worked together to create synchrony nearly all participants chose the cooperative option. For the other conditions, whether participants worked together or were in synchrony had no effect on the level of cooperation. The lack of any difference between the movement conditions in the individual goal condition was an interesting finding. This could suggest that shared intentionality is necessary to find a measurable synchrony-cooperation effect. Furthermore, the proportion of participants in the SqGG and AsGG was not higher than the SqIG and AsIG, suggesting that a shared goal by itself is not sufficient to increase cooperation either.

The path analysis supports the proposed combined path model incorporating both the joint attention model and the reinforcement of cooperation model. Synchrony and shared intentionality mutually reinforce each other both through the increased joint attention needed to create synchrony and through perceived synchrony being used as evidence for successful cooperation. The model also supports the idea that perceived similarity, interdependent selfconstrual and entitativity play a role in mediating the synchrony-cooperation effect -

\footnotetext{
${ }^{26} \mathrm{Yu}$ and Muthén (2001) suggest CFI > .95, RMSEA <.06, WRMR <.90 as cut off values for good models when the outcome variable is categorical (cited in Muthén, 2004).
} 
although due to these constructs being highly related, disentangling the specific causal route that each may play was not possible with this data.

The third aim of experiment 5 was to assess the relative importance of phase and entrainment. In terms of cooperative behaviour, the synchrony condition resulted in significantly more cooperation than both the asynchrony and sequential conditions. This suggests that being in-phase is important to produce an increase in cooperation. Although descriptive statistics indicate that the proportion of participants who choose the cooperative option was greater in the sequential condition than the asynchrony condition, this difference was not significant. This would seem to indicate that it is phase-locked synchrony rather than frequency-locked synchrony that fosters cooperation.

With the self-report variables, however, phase does not seem to hold the same importance. Although the sequential condition did produce a slightly lower mean level of entitativity and similarity, significant differences were only found between the synchrony and asynchrony conditions. Furthermore, the sequential condition produced a significantly higher level of entitativity than the asynchrony condition. This suggests that being frequency entrained even out of phase does increase people's social perception of being a group, although not quite to the same degree as being in-phase, and not enough it seems to influence behaviour.

\section{Chapter Discussion}

This chapter explored whether creating synchrony with shared intentionality resulted in larger and more robust increases in subsequent cooperation. Across all the experiments reported in this chapter, working together to create synchrony produced greater cooperation than comparison conditions. In particular, the importance of shared intentionality was emphasised by the finding in experiment 5 that being in synchrony only increased cooperation when people worked together to create synchrony. Significant differences between the synchrony and asynchrony conditions were also found with interdependent selfconstrual, entitativity and perceived similarity when movement was manipulated. No significant differences between these conditions were found in experiments 1, 2 and 3 where shared intentionality was not used to create synchrony. These results provide strong evidence in support of synchrony and shared intentionality combining to produce large, robust cooperation effects. 


\section{Chapter 6 \\ GENERAL DISCUSSION}

\section{Overview}

The overarching aim of this thesis was to contribute to explanations for why music and dance exists in the human species. In particular, I looked to gather evidence in support of the ultimate explanation that music and dance was selected through evolutionary processes because it provided the adaptive benefit of increasing the cooperative tendencies of a group. My approach to gathering such evidence was to concentrate on a specific feature of music and dance that appears across all cultures: namely, group synchrony. In order to understand the social cognitive and behavioural effects of group synchrony I isolated and manipulated group synchrony in the laboratory. I then tested the hypothesis that behaving in synchrony as a group would produce greater levels of cooperative behaviour. By isolating synchrony and measuring a number of potential psychological mediating factors, I was also able to examine how synchrony may foster cooperation. In this final chapter I discuss what evidence my five experiments provide in answering these two key questions of my thesis: does group synchrony increase cooperation, and if so, through what mechanism does it occur? I then assess what these results can tell us about music and dance as it occurs in natural human ecologies.

\section{Does group synchrony lead to greater cooperation?}

Results from four out of the five experiments I conducted offer support for the hypothesis that group synchrony leads to greater cooperation. Only in experiment 2, in which synchronous vocalising was used, did the synchrony condition fail to produce the highest mean level of cooperation. The overall finding that group synchrony produced greater levels of cooperation is in accordance with the results of Wiltermuth and Heath's (2009) experiments of group synchrony using a national anthem leading to greater cooperation. My studies extend Wiltermuth and Heath's findings by showing that even when cultural, social and emotional factors are minimised, synchrony still has an effect on cooperation. This is important as it provides evidence that synchrony is indeed a critical causal factor in 
increasing cooperation. My findings also link up with Valdesolo and DeSteno's (2011) finding that dyadic synchrony increases cooperation. My studies extend Valdesolo and DeSteno's work by showing that this effect also occurs with groups.

\section{The role of shared intentionality}

Although overall my results are supportive of group synchrony increasing cooperation, my experiments also highlight that how synchrony is created is important in influencing people's cooperative tendencies. In the first three experiments where synchrony was created incidentally through participants hearing the same beat, the effects appeared small and inconsistent. However, in experiments 4 and 5, where participants worked together to create synchrony, it was notable that significant differences between the synchrony and control conditions were consistently found. Examining across all studies, do the results suggest that shared intentionality is necessary for synchrony to enhance cooperation, or does shared intentionality accentuate synchrony's cooperative effects? The finding in experiment 5 that an increase in cooperation was limited to synchrony during the shared goal condition would appear to suggest that shared intentionality is necessary for cooperation. However, this conclusion does not square with the results of experiments 1 and 3, which found that synchrony affected cooperation without requiring shared intentionality. How can these results be integrated? Experiment 4 and 5 used a measure of cooperation that required social prediction - everybody is motivated to cooperate if they predict others would do the same. One possibility is that for cooperative situations that rely on anticipating the cooperative behaviours of others, the combination of synchrony and shared intentionality is particularly effective. Another possibility is that the individual nature of the synchrony manipulation in experiments 1 and 3 was not accentuated to the same degree as in the individual goal condition in experiment 5 . In all three experiments, participants were asked to move in time with the metronome beat and were made aware that the other participants would hear the same beat. In experiments 1 and 3, as participants in both experiments knew they were moving to the same beat they may not have wanted to move out of time out for fear of being perceived as doing the task incorrectly. Participants may then have paid more attention towards the other participants than in the asynchrony condition so as to try and keep in time. However, the use of the foot-pedals and video camera to monitor how well participants performed in experiment 5 may have lead participants in this experiment to particularly concentrate on the beat rather than the other participants. 
For synchronous movement, at least, it therefore appears plausible that shared intentionality is not necessary for synchrony to yield cooperation effects, however shared intentionality helps to accentuate such effects. This conclusion also fits with other studies on synchrony that found a significant increase in cooperation and social-perception measures with simple manipulations of the timing of movements without any shared goal (e.g., Valdesolo \& DeSteno, 2011). However, as no increase in cooperation was found with synchronous vocalising in experiment 2 , but an effect was found in experiment 4 , it could be that shared intentionality is necessary to produce a synchrony-cooperation effect with vocalising. In short, the behavioural modality of synchrony may be an important driver of cooperative modulation - an idea I will now discuss.

\section{The role of modality}

Most research and theorising about the cooperative effects of synchrony and mimicry have been based on body movements and perception-action system mediated by the visual system. Language obviously plays an important role in human bonding, but the ubiquity of group singing raises the prospect that the timing of people's vocalisations may also modulate cooperative tendencies. In my experiments I separated out movement and vocalisation and examined them separately. The finding in experiment 2 that the synchrony condition did not produce the highest level of synchrony initially suggested that the effect of synchrony may not extend to vocalisation. However, the results of experiment 4 showed that when participants worked together to create vocal synchrony, a significantly greater level of cooperation was found in the synchrony condition compared to the control condition. This indicates that simple group synchronous vocalising with emotionally neutral stimuli can indeed increase cooperation. Notably this interpretation dovetails with Wiltermuth and Heath's results that found that synchronous singing of a national anthem without any movement increased cooperation.

A challenge in examining the effects of synchronous vocalising that was highlighted in experiments 2 and 4, was that participants found asynchronous vocalising very difficult to perform. Creating an appropriate control condition proved particularly problematic in experiment 4 where there was no beat to guide participants. However, experiment 5 showed that intentionally moving at different frequencies to produce asynchrony was not impossible. Why was intentionally moving out of synchrony possible but not intentionally vocalising out of synchrony? One difference between movement and vocalisation synchrony described in 
chapter 4 (see page 51) is that visual information is much more focused than auditory information. With asynchronous movement, participants could control the visual information they received by averting their gaze. They were therefore able to avert their attention from any distracting asynchronous rhythms. The attention questions from experiment 5 indicated that participants in the asynchrony group goal condition did indeed pay less attention to the other participants than in the synchrony and sequential conditions. Auditory information, on the other hand, is more difficult to control. A person cannot as easily avert their ears from a sound apart from trying to physically prevent the sound from entering the ears (such as putting one's hands over one's ears).

Because of fundamental differences in how humans' perceptual systems work with visual and auditory information as well as differences in the social uses of vocalisation and movements, there are likely to be differences in the social effects of synchronous movement and synchronous vocalising. Both synchronous movement and synchronous vocalising do appear to influence cooperative behaviour, but it is not clear if they do so to the same degree or through the same mechanisms. Further investigations into the differences between vocal and movement synchrony could be an important direction for future research. A better understanding of vocal synchrony may also help link human coordinated vocalising to coordinated vocalising seen in other species (e.g., duetting in gibbons; see page 19).

\section{The role of phase}

As outlined in chapter 2, synchrony can be defined as frequency-locked or phaselocked. In experiment 4, I compared phase-locked synchrony to a condition in which participants were frequency-locked but out of phase (sequential condition). I found a significant difference between these two conditions indicating that phase is important in influencing cooperation. In experiment 5 I directly compared these two types of entrainment. The finding of significant differences between the synchrony condition and the sequential and asynchrony conditions in the proportions that cooperate, support the results from experiment 4 that phase does influence people's cooperative tendencies. The non-significant difference in cooperation between the sequential and asynchrony conditions in experiment 5 suggests that out-of-phase entrainment does not have the same impact on cooperative tendencies. However, this does not necessarily mean that out-of-phase synchrony has no effect at all on cooperation. The sequential condition did result in a slightly higher proportion of participants cooperating than the asynchrony, and moreover, the mean level of entitativity for the 
sequential condition was significantly higher compared to the asynchrony condition. This supports the notion that out-of-phase synchrony does produce some prosocial effect.

Are the cooperative effects limited to the in-group?

Assessing if group synchrony increases cooperation may also be dependent on who participants are cooperative towards. This question was examined in experiments 1 and 2 . Both studies found no differences in cooperative tendencies directed towards a fellow group member and towards a non-performance member. For experiment 1 where some indication of a synchrony-cooperation effect was found, this supports the idea that synchrony results in a more generalised prosocial inclination. However, the finding of significant differences in entitativity between movement conditions in experiments 3 and 5 might raise doubts over this conclusion. Data from minimal group studies suggest that an increase in group identity produces an in-group bias: people are more likely to cooperate with a fellow group member (Hewstone et al., 2002; Mullen et al., 1992). Such in-group bias does not necessarily result in discrimination against out-group members though (Brewer, 1999; Yamagishi \& Mifune, 2009). One possibility is that synchrony may increase cooperation towards a stranger (through say a shift in generalised interdependent self-construal); however, increased entitativity results in a larger increase towards a fellow performance member. Because of potential confounds in the measurement of cooperation towards a fellow group member in experiments 1 and 2 (see page 60), one possibility to further examine the limits of the cooperation effect of synchrony could be to replicate experiment 5 with a generalised cooperation measure such as donating to a charity. A future study in which group identity as well as synchrony is also manipulated through the minimal group paradigm (Billig \& Tajfel, 1973) could also be informative. Examining the cooperative effects of out-group members may be especially important given the use of group synchrony by armies and fascist regimes - groups that are associated with hostility towards out-group members. There is also some experimental evidence to suggest that group synchrony may result in aggressive behaviour towards out-group members through increased compliance to a suggestion from another group member (Wiltermuth, 2012). 


\section{How does synchrony increase cooperation?}

In addition to examining whether group synchrony increases cooperation, my second major research question focussed on the mediating mechanisms by which group synchrony produced this effect. In this thesis I explored the potential mediating role of perceived similarity, interdependent self-construal and entitativity. In experiment 1 and 2, I found no significant differences with any of these measures across conditions. In experiment 3, I did find a significant difference between the synchrony and passive condition in entitativity, but only a small, non-significant difference between the synchrony and asynchrony conditions. However, entitativity in experiment 3 was not correlated with cooperation. When shared intentionality was introduced, significant differences between conditions were found with perceived similarity, interdependent self-construal and entitativity. Experiment $3 b$ found that combining synchrony with shared intentionality produced significantly greater levels of interdependent self-construal targeted at the performance group than the other conditions, with interdependent self-construal being significantly correlated with cooperation. In experiment 5 both similarity and entitativity was significantly greater in the synchrony than in the asynchrony condition, with both measures being significantly correlated with cooperation. This provides preliminary evidence supporting the role of these measures in producing the cooperation effect.

Further support for the role of perceived similarity, interdependent self-construal and entitativity was acquired through the path analysis in experiment 5 . The model provided a very good fit for the data, confirming that a combined measure of perceived similarity, interdependent self-construal and entitativity (what I termed 'social cohesion') mediated the relationship between synchrony (created through shared intentionality) and cooperation. Path analysis also supported the combination of the two theoretical mechanisms I proposed: the joint attention model and the reinforcement of cooperation model. Therefore, as well as describing a detailed theoretical account for how group synchrony and shared intentionality might combine to increase cooperation in this thesis, I provided valuable empirical data supporting these models.

\section{Limitations of the study and future research}

Although I found that manipulating synchrony did result in greater cooperation, it is important to emphasise that performing in synchrony does not guarantee cooperation. This is 
both due to individual differences, cultural differences, and situational factors. Although my results showed that, on average, people cooperated more, not everybody in the synchrony condition chose to cooperate. Personality factors such as social value orientation (e.g., De Cremer, van Knippenberg, van Dijk, \& van Leeuwen, 2008) and trait self-construal (Markus \& Kitayama, 1991) might influence how effective synchrony is in influencing participants' cooperative tendencies. Other factors such as a participant's mood or stress levels at the time the experiment was conducted may also be important (Batson, 1998). While random assignment to conditions mean that individual variability will average out, it would be interesting to investigate the causes of variation from the mean. Although this study cannot predict with any high degree of certainty if any particular individual will cooperate depending on how synchronised he/she was, it does tell us, all things being equal, that, on average, participants will be more likely to cooperate after group synchronous behaviour.

Another limitation of this study is the effects I found were quite small. This raises the question as to whether the increase in cooperation is large enough to warrant the cost of the energy expended in performing the behaviour. However, it is important to keep in mind that synchrony in these experiments was created under very minimal conditions. In group synchronous performance in natural ecologies there are a number of factors that may accentuate the effect synchrony has on cooperation. For example, most natural expression of music and dance last considerably longer than the six minutes used in the experiments in this thesis, sometimes continuing for hours (D. A. Marshall, 2002; Merriam, 1964; Rossano, 2009). Music and dance performances throughout most of human history would have also likely been performed by groups of people who knew each other well, rather than a small group of strangers as used in my experiments. The effects of synchrony may be even stronger with existing groups for which a group identity already exists and for which synchrony can then accentuate. Group synchronous performance in natural ecologies also often involves other techniques that help accentuate group identity such as similar dress, a shared cultural meaning of the dance or music, and lyrics that prime unique attributes of the group (Boer \& Fischer, 2012; Giles, Denes, Hamilton, \& Hajda, 2009; Hanna, 1979). The experiments in this thesis highlight that group synchrony could be one important factor in group music and dance in influencing cooperative tendencies. Future studies can build on the findings contained herein to examine the interaction of synchrony with other key variables involved in music and dance. In particular, it would be important in future research to investigate how these different factors combine in natural ecologies, as our own research group has started to explore (Fischer, Callander, Reddish, \& Bulbulia, 2012). 
The mechanism I proposed for how synchrony increases cooperation only involved the measurement of psychological constructs. This restricted the scope for explanations of how group synchrony increases cooperation. Other avenues of research have started to explore the effects of synchrony at different levels of explanation, in particular at the neural level. As suggested in chapter 4, mirroring brain processes incorporated into perceptionaction neural systems may be involved in generating the synchrony-cooperation effect. Neuroimaging (e.g., Iacoboni et al., 1999) and established experimental protocols such as the automatic imitation paradigm (Heyes, Bird, Johnson, \& Haggard, 2005) could be used to assess if synchrony activates the mirror neuron system and if such activation correlates with cooperative behaviours (Shaw, 2012). Particular neurotransmitters, for example oxytocin, have also been suggested to be involved in generating cooperation from music and dance (Freeman, 2000). Importantly, oxytocin has been found to lead to greater trust and generosity towards others (Zak, Kurzban, \& Matzner, 2005; Zak, Stanton, \& Ahmadi, 2007), although, how music, dance or synchrony could produce greater oxytocin is not clear. Endorphins have also been implicated in mediating the synchrony-cooperation effect. Endorphins are endogenous opioids that create feelings of well-being and have been linked to social bonding in primates (Graves, Wallen, \& Maestripieri, 2002) and implicated for social bonding in humans (Dunbar, 2010). A study by Cohen, Ejsmond-Frey, Knight, and Dunbar (2009) found that rowing in time with others compared to rowing alone resulted in greater pain threshold suggesting endorphin release. However, it was not clear from this study if it was synchrony per se or some other social factor that produced that elevated pain thresholds. As with oxytocin, it is also not clear what about synchrony may produce an endorphin release, nor is it clear how endorphins may affect cooperation. Further evidence of reward systems being involved in the synchrony-cooperation effect comes from a functional magnetic resonance imaging (fMRI) study (Kokal, Engel, Kirschner, \& Keysers, 2011). This study found increased activation in areas of the brain involved in processing rewards (caudate nucleus) after synchronous drumming. Moreover, the degree of activation in the caudate nucleus was correlated with increased prosocial behaviour (the number of spilled pencils a participant picked up) measured after the scanning. The authors suggested that the reward generated from successfully synchronising became associated with the co-drummer. This reward association then became activated when given an opportunity to help the co-drummer. This idea links nicely with the reinforcement of cooperation model proposed in chapter 5 . The caudate nucleus is particularly innervated by dopaminergic connections also suggesting a possible role for dopamine in mediating the reward effects of synchronous drumming (Schott 
et al., 2008). Although the relationship between dopamine and synchrony has yet to be investigated, dopamine release in the striatum (which includes the caudate nucleus) has been found during peak emotional experiences from music listening (Salimpoor, Benovoy, Larcher, Dagher, \& Zatorre, 2011).

\section{Generalisability of the results}

A crucial question for the results I found to be able to say something meaningful about the origins of music and dance, is how well do the results generalise to the real world? In particular, did my synchrony manipulation correspond within a reasonable degree to natural expressions of music and dance? Research from Lomax and collaborators (Lomax, 1968) found that music and dance varies in the degree and type of synchrony expressed, i.e., some expressions involve a group performing exactly the same movement or vocalisation at the same time (phase-locked synchrony), other expressions involve people performing different actions but still entrained to the same underlying beat (frequency-locked synchrony). In both experiment 4 and experiment 5 , I found that in-phase synchrony resulted in a significantly greater proportion of participants cooperating than out of phase but frequency-locked movement. If in-phase synchrony does result in more cooperation than frequency-locked entrainment, why do not all performances of music and dance involve inphase synchrony? One possibility was that my manipulation of frequency-locked synchrony was not a good representation of the complex inter-personal phase patterning that is commonly found in music and dance. For example, in the sequential condition, participants performed and then stood or sat passively while the other group members performed. This may have made people more self-aware as only one participant was performing at a time, so increasing their feelings of being an individual. An increase in cooperation may have been found if participants were continuously performing out of phase with each other.

Another possibility is that the form a particular music and dance performance takes is the product of a balance between a variety of human needs. For example, according to optimal distinctiveness theory, people possess the conflicting needs of affiliation and differentiation from others (Brewer, 1991). People want to belong, but desire to feel unique as well. More complex forms of music and dance may be a compromise between these two opposing needs. Performing in synchrony creates affiliation, with a performers' own unique contribution to the collective performance satisfying a person's need to feel distinctive. Certain music and dance performances may have also developed to cater for other human 
needs so as to keep people motivated to perform them. Performing simple movements in time with others for hours, performers may find boring. Human's desire for novelty and mental stimulation may have driven music and dance to develop more complex forms, so as to again find a balance between the social benefit of performing in time, and the individual benefit of being engaged in the task.

Music and dance with complex phase patterns also require more practice and careful coordination to successfully perform them (compare an orchestra playing Beethoven to a crowd chanting at a football game) (Giles et al., 2009). The numerous hours of practice needed to successfully produce complex musical and dance group performances results in more time spent in synchrony and so could result in increased group identity and so greater cooperation. Moreover, because more complex forms of synchrony are more difficulty to do, when a group does successfully pull off the desired performance this could result in more pronounced feelings of success as a group and so produce greater cooperative effects (through the reinforcement of cooperation route) than a simple easy to perform song or dance.

It is also important to bear in mind that music and dance are continually changing through cultural evolution. That is certain forms of music and dance we see now, may not be representative of music and dance as they have been performed for most of our history. Exploration of the cultural evolution of song styles, for example using phylogenetic trees (c.f. Gray \& Atkinson, 2003 with language evolution), may give clues as to what ancestral songs and dances may have been like (Leroi \& Swire, 2006). If simple group phase-locked synchronous vocalisations and movement were found to be an ancient form of song and dance, this would certainly strengthen the claim that the creation of group synchrony was an important factor in the evolution of music and dance.

\section{Final conclusions}

In summary, over the five social psychology experiments I conducted, I found replicable evidence that group synchrony increases cooperation even under minimal conditions. In particular, this thesis highlighted the role that shared intentionality plays in influencing this effect. Working together to create synchronous movement or synchronous vocalising produced large reliable increases in cooperation and perceived cohesion with others. The importance of shared intentionality combined with synchrony in producing the cooperative effects of music and dance performances dovetails with research by Tomasello 
and collaborators who argue that shared intentionality played a vital role in the evolution of human sociality. I also highlighted that the modality of the synchrony and the phase relationship of the synchrony influences the synchrony-cooperation effect. Lastly, in this thesis I provided detailed theoretical mechanisms for how synchrony may increase cooperation, and furthermore, I gathered empirical evidence supporting these mechanisms.

Although this project cannot explain all of the variance found in the category of behaviours we commonly term music and dance, the results do supply evidence supporting the importance of synchrony in creating the cooperative effects of music and dance. This in turn supports the hypothesis that music and dance developed in the human species due to the cooperative benefits it brought our ancestors. Such research has important practical implications. A number of authors have suggested that group synchrony played a vital role in the creation of society (I. Cross, 1999; Durkheim, 1965; Ehrenreich, 2006), with McNeill (1995) going so far as to state that "large and complex human societies, in all probability, cannot maintain themselves without such kinaesthetic undergirding" (p. 152). Synchrony's powerful bonding mechanism, it seems, has the potential to be used for anti-social purposes when combined with negative values towards others, for example producing compliant cohesive groups willing to aggress against out-group members (e.g., tightly drilled cohesive army units, see Wiltermuth, 2012). However, synchrony also has the potential to be used for great good. Policy could support rhythmic group practices as a way of increasing cooperation, trust, love and happiness in society (Haidt et al., 2008; Peretz, 2006). For example musician Brian Eno (2008), in a recent interview on National Public Radio (NPR), suggested: "I believe in singing to such an extent that if I were asked to redesign the British educational system, I would start by insisting that group singing become a central part of the daily routine. I believe [group singing] ... more than anything else, encourages a taste for cooperation with others". Corporations could include synchronous group activities to help with team building and cooperation amongst workers to increase output (e.g., calisthenics used by some Japanese corporations, Powell, 2009). Moreover, if synchronous activities help to shift people from being self-focused to a more interdependent-self which is part of something bigger, it could aid in world-wide coordination and cooperation problems such as the sustainability of resources and the health of the planet. Used wisely then, singing and dancing together could be a wonderful tool to bring together humanity. 


\section{References}

Adler, D. S. (2009). The earliest musical tradition. Nature, 460(7256), 695-696. doi:10.1038/460695a

Andreoni, J. (1995). Cooperation in public-goods experiments: kindness or confusion? American Economic Review, 85, 891-904.

Anshel, A., \& Kipper, D. A. (1988). The influence of group singing on trust and cooperation. Journal of Music Therapy, 25(3), 145-155.

Arom, S. (1991). African polyphony and polyrhythm, musical structure and methodology. Cambridge, U.K: Cambridge University Press.

Aron, A., Aron, E. N., \& Smollan, D. (1992). Inclusion of other in the self scale and the structure of interpersonal closeness. Journal of Personality and Social Psychology, 63(4), 596-612.

Aronson, E., \& Mills, J. (1959). The effect of severity of initiation on liking for a group. Journal of Abnormal and Social Psychology, 59(2), 177-181.

Ashton-James, C., van Baaren, R. B., Chartrand, T. L., Decety, J., \& Karremans, J. (2007). Mimicry and me: The impact of mimicry on self-construal. Social Cognition, 25(4), 518-535.

Backwell, P., Jennions, M., Passmore, N., \& Christy, J. (1998). Synchronized courtship in fiddler crabs. Nature, 391, 31-32.

Barrett, L. F., \& Russell, A. A. (1999). The structure of current affect: Controversies and emerging consensus. Current Directions in Psychological Science, 8(1), 10-14.

Barsalou, L. W., Niedenthal, P. M., Barbey, A., \& Ruppert, J. (2003). Social embodiment. In B. Ross (Ed.), The psychology of learning and motivation (pp. 43-92). San Diego: Academic Press.

Batson, C. D. (1998). Altruism and prosocial behavior. In D. Gilbert, S. T. Fiske, \& G. Lindzey (Eds.), The handbook of social psychology (Vol. 2, pp. 282-316). New York: McGraw-Hill.

Bavelas, J. B., Black, A., Lemery, C. R., Macinnis, S., \& Mullett, J. (1986). Experimental methods for studying elementary motor mimicry. Journal of Nonverbal Behavior, 10(2), 102-119.

Bellah, R. N. (2003). The ritual roots of society and culture. In M. Dillon (Ed.), Handbook of the sociology of religion (pp. 31-44). Cambridge, UK: Cambridge University Press.

Bennett, M., Schatz, M. F., Rockwood, H., \& Wiesenfeld, K. (2002). Huygens's clocks. Series A: Mathematical Physical and Engineering Sciences, 458, 563-579. 
Benzon, W. (2001). Beethoven's anvil: Music in mind and culture. New York: Basic Books.

Berghout-Austin, A. M., \& Peery, J. C. (1983). Analysis of adult-neonate synchrony during speech and non speech. Perceptual Motor Skills, 57, 455-459.

Bernieri, F. J. (1988). Coordinated movement and rapport in teacher-student interactions. Journal of Nonverbal Behavior, 12(2), 120-138.

Bernieri, F. J., Davis, J. M., Rosenthal, R., \& Knee, C. R. (1994). Interactional synchrony and rapport - measuring synchrony in displays devoid of sound and facial affect. Personality and Social Psychology Bulletin, 20(3), 303-311.

Bernieri, F. J., \& Rosenthal, R. (1991). Interpersonal coordination: Behavior matching and interactional synchrony. In R. S. Feldman \& B. Rime (Eds.), Fundamentals of nonverbal behavior: Studies in emotion \& social interaction (pp. 401-432). New York: Cambridge University Press.

Billig, M., \& Tajfel, H. (1973). Social categorization and similarity in intergroup behaviour. European Journal of Social Psychology, 3(1), 27-52.

Binmore, K. (1998). Game theory and the social contract: Just playing. Cambridge, MA: MIT Press.

Binmore, K. (2008). Do conventions need to be common knowledge? Topoi, 1, 17-27.

Bispham, J. (2006). "Music" means nothing if we don't know what it means. Journal of Human Evolution, 50, 587-593.

Bispham, J. (2010). Music's “design features”: Musical motivation, musical pulse, and musical pitch. Musicae Scientiae, (2009), 29-44.

Blacking, J. (1973). How musical is man. Seattle: University of Washington Press.

Blacking, J. (1995). Music, culture and experience. London: University of Chicago Press.

Blanke, O., \& Metzinger, T. (2009). Full-body illusions and minimal phenomenal selfhood. Trends in Cognitive Sciences, 13, 7-13.

Boas, F. (1972). Dance and music in the life of the northwest coast indians of North America (Kwakiutl). In F. Boas (Ed.), The function of dance in human society (2nd ed., pp. 1-20). New York: Dance Horizons.

Boer, D., \& Fischer, R. (2012). Towards a holistic model of functions of music listening across cultures: A culturally decentred qualitative approach. Psychology of Music, 40(2), 179-200. doi:10.1177/0305735610381885

Boer, D., Fischer, R., Tekman, H. G., Abubakar, A., Njenga, J., \& Zenger, M. (2012). Young people's topography of musical functions: Personal, social and cultural experiences with music across genders and six societies. International Journal of Psychology, 37-41. doi:10.1080/00207594.2012.656128 
Botvinick, M., \& Cohen, J. D. (1998). Rubber hand "feels" what eyes see. Nature, 391, 756.

Bowles, S. (2009). Did warfare among ancestral hunter-gatherers affect the evolution of human social behaviors? Science, 324(5932), 1293-1298. doi:10.1126/science.1168112

Boyd, R., \& Richerson, P. J. (2010). Transmission coupling mechanisms: Cultural group selection. Philosophical Transactions of the Royal Society B-Biological Sciences, 37873795. doi:10.1098/rstb.2010.0046

Boyd, R., Richerson, P. J., \& Henrich, J. (2011). The cultural niche: Why social learning is essential for human adaptation. Proceedings of the National Academy of Sciences, 108, 10918-10925. doi:10.1073/pnas.1100290108

Boyer, P., \& Lienard, P. (2006). Why ritualized behavior? Precaution systems and action parsing in developmental, pathological and cultural rituals. Behavioral and Brain Sciences, 29(6), 595-650. doi:10.1017/s0140525x06009332

Bradley, M. M., \& Lang, P. J. (1999). Affective norms for English words (ANEW): Instruction manual and affective ratings. Technical Report C-1. Gainesville, FL: The Center for Research in Psychophysiology, University of Florida.

Bressan, P., Generale, P., \& Padova, U. (2009). Biologically costly altruism depends on emotional closeness among step but not half or full sibling. Evolutionary Psychology, 7(1), 118-132.

Brewer, M. B. (1991). The social self: On being the same and different at the same time. Personality and Social Psychology Bulletin, 17(5), 475-482. doi:10.1177/0146167291175001

Brewer, M. B. (1999). The psychology of prejudice: Ingroup love or outgroup hate? Journal of Social Issues., 55(3), 429-444.

Brown, D. (1991). Human universals. New York: McGraw-Hill.

Brown, S. (2000). Evolutionary models of music: From sexual selection to group selection. In F. Tonneau \& N. S. Thompson (Eds.), Perspectives in ethology 13: Behavior, evolution and culture (pp. 231- 281). New York: Plenum Publishers.

Bulbulia, J. (2012). Spreading order: religion, cooperative niche construction, and risky coordination problems. Biology and Philosophy, 27, 1-27. doi:10.1007/s10539-0119295-x

Bulbulia, J., \& Reddish, P. (2012). Explaining effervescence. In G. Dawes \& J. McClaurin, (Eds.), A New Science of Religion, (pp. 43-64). New York: Routledge.

Buller, D. J. (2005). Adapting minds: Evolutionary psychology and the persistent quest for human nature. Cambridge, MA: MIT Press.

Calcott, B. (2008). The other cooperation problem: generating benefit. Biology and Philosophy, 23, 179-203. 
Campbell, D. (1958). Common fate, similarity, and other indices of the status of aggregates of persons as social entities. Behavioral Science, 3(1), 14-25.

Cappella, J. N., \& Planalp, S. (1981). Talk and silence sequences in informal conversations III: Interspeaker influence. Human Communication Research, 7, 117-132.

Castano, E., Yzerbyt, V., \& Bourguignon, D. (2003). We are one and I like it: The impact of ingroup entitativity on ingroup identification. European Journal of Social Psychology, 33(6), 735-754. doi:10.1002/ejsp.175

Charney, E. J. (1966). Psychosomatic manifestations of rapport in psychotherapy. Psychosomatic Medicine, 28, 305-315.

Chartrand, T. L., \& Bargh, J. A. (1999). The Chameleon effect: The perception-behavior link and social interaction. Journal of Personality and Social Psychology, 76(6), 893-910.

Chartrand, T. L., \& van Baaren, R. (2009). Human mimicry. Advances in experimental social psychology (Vol. 41, pp. 219-274). San Diego: Elsevier Academic Press.

Clayton, M. (2009). The social and personal functions of music in cross-cultural perspective. In S. Hallam, I. Cross, \& M. Thaut (Eds.), The Oxford handbook of music psychology (pp. 35-44). New York, NY: Oxford University Press.

Clayton, M., Sager, R., \& Will, U. (2004). In time with the music: The concept of entrainment and its significance for ethnomusicology. CounterPoint, 1, 1-45.

Coats, S., Smith, E. R., Claypool, H., \& Banner, M. (2000). Overlapping mental representations of self and in-group: Reaction time evidence and its relationship with explicit measures of group identification. Journal of Experimental Social Psychology, 36(3), 304-315. doi:10.1006/jesp.1999.1416

Cohen, E. E. A., Ejsmond-Frey, R., Knight, N., \& Dunbar, R. I. M. (2010). Rowers' high: behavioural synchrony is correlated with elevated pain theshholds. Biology Letters, 6, 106-108.

Conard, N. J., Malina, M., \& Munzel, S. C. (2009). New flutes document the earliest musical tradition in southwestern Germany. Nature, 460(7256), 737-740. doi:10.1038/nature08169

Condon, W. S., \& Ogston, W. D. (1967). A segmentation of behavior. Journal of Psychiatric Research, 5, 221-235.

Condon, W. S., \& Sander, L. W. (1974). Synchrony demonstrated between movements of the neonate and adult speech. Child Development, 45(2), 456-462.

Croson, R. (2005). The method of experimental economics. International Negotiation, 10, 131-148. 
Cross, I. (1999). Is music the most important thing we ever did? Music, development and evolution. In S. Yi (Ed.), Music, mind and science (pp. 10-39). Seoul: Seoul National University press.

Cross, I. (2001). Music, cognition, culture, and evolution. Annals of the New York Academy of sciences, (930), 28-42.

Cross, I. (2003). Music and biocultural evolution. In M. Clayton, T. Herbert, \& R. Middleton (Eds.), The cultural study of music: A critical introduction (pp. 19-30). London: Routledge.

Cross, I. (2009). The nature of music and its evolution. In Susan Hallam, I. Cross, \& M. H. Thaut (Eds.), The Oxford handbook of music psychology (pp. 3-13). New York: Oxford University Press.

Cross, S. E., Bacon, P. L., \& Morris, M. L. (2000). The relational-interdependent selfconstrual and relationships. Journal of Personality and Social Psychology, 78(4), 791808. doi:10.1037//0022-3514.78.4.791

Cross, S. E., Hardin, E. E., \& Gercek-Swing, B. (2011). The what, how, why, and where of self-construal. Personality and Social Psychology Review, 15(2), 142-79. doi:10.1177/1088868310373752

Cummins, F. (2009). Rhythm as entrainment: The case of synchronous speech. Journal of Phonetics, 37, 16-28.

Darwin, C. (1871). The descent of man, and selection in relation to sex. London: John Murray. Retrieved from http://darwin-online.org.uk/pdf/1871_Descent_F937.1.pdf

Dawes, R. M., Van de Kragt, A. J., \& Orbell, J. M. (1988). Not me or thee but we: The importance of group identity in eliciting cooperation in dilemma situations: Experimental manipulations. Acta Psychologica, 68, 83-97.

De Cremer, D., van Knippenberg, D., van Dijk, E., \& van Leeuwen, E. (2008). Cooperating if one's goals are collective-based: Social identification effects in social dilemmas as a function of goal transformation. Journal of Applied Social Psychology, 38(6), 15621579.

Di Pellegrino, G., Fadiga, L., Fogassi, L., Gallese, V., \& Rizzolatti, G. (1992). Understanding motor events: a neurophysiological study. Experimental Brain Research, 91, 176-180.

Dilley, R. M. (2002). The problem of context in social and cultural anthropology. Language \& Communication, 22, 437-456.

Dittmann, A. T., \& Llewellyn, L. G. (1969). Body movement and speech rhythm in social conversation. Journal of Personality and Social Psychology, 11(2), 98-106.

Dummer, T., Picot-Annand, A., Neal, T., \& Moore, C. (2009). Movement and the rubber hand illusion. Perception, 38(2), 271-280. doi:10.1068/p5921 
Dunbar, R. I. M. (2010). The social role of touch in humans and primates: behavioural function and neurobiological mechanisms. Neuroscience and Biobehavioral Reviews, 34(2), 260-8. doi:10.1016/j.neubiorev.2008.07.001

Dunbar, R. I. M., \& Shultz, S. (2010). Bondedness and sociality. Behaviour, 147(7), 775-803. doi:10.1163/000579510x501151

Durkheim, E. (1965). The elementary forms of the religious life (J. W. Swain, Trans.). New York: Free Press. (Original work published 1915). Retrieved from http://www.archive.org/stream/elementaryformso00durk\#page/n1/mode/2up

Ehrenreich, B. (2006). Dancing in the streets: A history of collective joy. New York: Metropolitan.

Ellis, H. (1923). The dance of life. New York: Houghton Mifflin.

Evans-Pritchard, E. E. (1928). The Dance. Africa: Journal of the International African Institute, 1(4), 446-462.

Fischbacher, U. (2001). Are people conditionally cooperative? Evidence from a public goods experiment. Economics Letters, 71(3), 397-404. doi:10.1016/S0165-1765(01)00394-9

Fischer, R., Callander, R., Reddish, P., \& Bulbulia, J. (2012). How do rituals affect cooperation? An experimental field study comparing nine ritual types. Manuscript submitted for publication.

Fisher, N.I. (1993). Circular Statistics. Cambridge, UK: Cambridge University Press.

Fitch, W. T. (2006). The biology and evolution of music: A comparative perspective. Cognition, 100(1), 173-215. doi:10.1016/j.cognition.2005.11.009

Freeman, W. J. (2000). A neurobiological role of music in social bonding. In N. L. Wallin, B. Merker, \& S. Brown (Eds.), The origins of music (pp. 411-424). Cambridge, MA: MIT Press.

Friedman, D., \& Sunder, S. (1994). Experimental methods: A primer for economists. New York : Cambridge University Press.

Gallistel, C. R. (1980). The organization of action: A new synthesis. Hillsdale, NJ: Erlbaum.

Gardner, W. L., Gabriel, S., \& Lee, A. Y. (1999). "I" value freedom, but "we" value relationships: Self-construal priming mirrors cultural differences in judgment. Psychological Science, 10(4), 321-326.

Geissmann, T. (2000). Gibbon songs and human music from an evolutionary perspective. In N. Wallin, B. Merker, \& S. Brown (Eds.), The origins of music (pp. 103-124). Cambridge, MA: MIT Press. 
Giles, H., Denes, A., Hamilton, D. L., \& Hajda, J. M. (2009). Striking a chord: A prelude to music and intergroup relations research. Group Processes \& Intergroup Relations, 12(3), 291-301. doi:10.1177/1368430209102840

Glass, L., \& Mackey, M. C. (1988). From clocks to chaos: The rhythms of life. Princeton: Princeton University Press.

Gore, J. S., \& Cross, S. E. (2006). Pursuing goals for us: Relationally autonomous reasons in long-term goal pursuit. Journal of Personality and Social Psychology, 90, 848-861. doi:doi:10.1037/0022-3514.90.5.848

Gorer, G. (1972). Function of dance forms in primitive African communities. In Franziska Boas (Ed.), The function of dance in human society (2nd ed., pp. 21-40). New York: Dance Horizons.

Gourlay, K. A. (1984). The non-universality of music and the universality of non-music. The World of Music, 26, 25-36.

Graves, F. C., Wallen, K., \& Maestripieri, D. (2002). Opioids and attachment in rhesus macaque (Macaca mulatta) abusive mothers. Behavioral Neuroscience, 116(3), 489-493. doi:10.1037//0735-7044.116.3.489

Gray, R. D., \& Atkinson, Q. D. (2003). Language-tree divergence times support the Anatolian theory of Indo-European origin. Nature, 426, 435-439.

Greenfield, M. D. (1994). Cooperation and conflict in the evolution of signal interactions. Annual Review of Ecological Systems, 25, 97-126.

Greitemeyer, T. (2009). Effects of songs with prosocial lyrics on prosocial thoughts, affect, and behavior. Journal of Experimental Social Psychology, 45(1), 186-190. doi:10.1016/j.jesp.2008.08.003

Griffiths, P. E., \& Gray, R. D. (2005). Three ways to misunderstand developmental systems theory. Biology and Philosophy, 20, 417-425.

Grosse, E. (1897). The beginnings of art. New York: D. Appleton and Company. Retrieved from http://archive.org/stream/beginningsofart00grosuoft\#page/n7/mode/2up

Gueguen, N. (2009). Mimicry and seduction: An evaluation in a courtship context. Social Influence, 4(4), 249-255.

Haberstroh, S., Oyserman, D., Schwarz, N., Kühnen, U., \& Ji, L. (2002). Is the interdependent self more sensitive to question context than the independent self? Selfconstrual and the observation of conversational norms. Journal of Experimental Social Psychology, 38, 323-329. doi:doi:10.1006/jesp.2001.1513

Hadar, U., Steiner, T. J., \& Rose, F. C. (1985). Head movement during listening turns in conversation. Journal of Nonverbal Behavior, 9, 214-228. 
Hagen, E. H., \& Bryant, G. A. (2003). Music and dance as a coalition signaling system. Human Nature, 14(1), 21-51.

Haidt, J. (2007). The new synthesis in moral psychology. Science, 316(5827), 998-1002. doi:10.1126/science.1137651

Haidt, J., Seder, J. P., \& Kesebir, S. (2008). Hive psychology, happiness, and public policy. Journal of Legal Studies, 37, S133-S156. Chicago, IL: Univ Chicago Press.

Haley, K. J., \& Fessler, D. M. T. (2005). Nobody's watching? Subtle cues affect generosity in an anonymous economic game. Evolution and Human Behavior, 26(3), 245-256. doi:10.1016/j.evolhumbehav.2005.01.002

Hamilton, W. D. (1964). The genetical evolution of social behaviour I and II. Journal of Theoretical Biology, 7, 1-52.

Hanna, J. L. (1977). African dance and the warrior tradition. Journal of Asian and African Studies, 12, 111-133.

Hanna, J. L. (1979). Movements toward understanding humans through the anthropological study of dance. Current Anthropology, 20(2), 313-339.

Hanna, J. L. (1987). To dance is human : A theory of nonverbal communication. Chicago: University of Chicago Press.

Harb, C., \& Smith, P. B. (2008). Self-construals across cultures - Beyond independenceinterdependence. Journal of Cross-Cultural Psychology, 39(2), 178-197. doi:10.1177/0022022107313861

Hardin, G. (1968). The Tragedy of the commons. Science, 162, 1243-1248.

Hauser, M. D., \& McDermott, J. (2003). The evolution of the music faculty: a comparative perspective. Nature Neuroscience, 6(7), 663-668. doi:10.1038/nn1080

Hayes, D. P., \& Cobb, L. (1982). Cycles of spontaneous conversation under longterm isolation. In M. Davis (Ed.), Interaction rhythms: Periodicity in communicative behavior (pp. 319-340). New York: Human Science Press.

Hewstone, M., Rubin, M., \& Willis, H. (2002). Intergroup bias. Annual Review of Psychology, 53, 575-604.

Heyes, C. M., Bird, G., Johnson, H., \& Haggard, P. (2005). Experience modulates automatic imitation. Cognitive Brain Research, 22, 233-240.

Houser, D., \& Kurzban, R. (2002). Revisiting kindness and confusion in public goods experiments. American Economic Review, 92(4), 1062-1069.

Hove, M. J. (2008). Shared circuits, shared time, and interpersonal synchrony. Behavioral and Brain Sciences, 31(1), 29-30. doi:10.1017/s0140525x07003202 
Hove, M. J., \& Risen, J. L. (2009). It's all in the timing: Interpersonal synchrony increases affiliation. Social Cognition, 27(6), 949-960.

Hurley, S. (2005). The Shared circuits hypothesis: A unified functional architecture for control, imitation, and simulation. In S. Hurley \& N. Chater (Eds.), Perspectives on imitation: From neuroscience to social science. Cambridge: MA: MIT Press.

Hurley, S., Clark, A., \& Kiverstein, J. (2008). The shared circuits model (SCM): How control, mirroring, and simulation can enable imitation, deliberation, and mindreading. Behavioral and Brain Sciences, 31(1), 1-58. doi:10.1017/s0140525x07003123

Huron, D. (2001). Is music an evolutionary adaptation? Annals of the New York Academy of sciences, 930, 43- 61.

Iacoboni, M. (2009). Imitation, empathy, and mirror neurons. Annual Review of Psychology, 60, 653-670. doi:10.1146/annurev.psych.60.110707.163604

Iacoboni, M., Woods, R. P., Brass, M., Bekkering, H., Mazziotta, J. C., \& Rizzolatti, G. (1999). Cortical mechanisms of human imitation. Science, 286, 2526-2528.

Issartel, J., Marin, L., \& Cadopi, M. (2007). Unintended interpersonal co-ordination: "can we march to the beat of our own drum?". Neuroscience letters, 411(3), 174-9. doi:10.1016/j.neulet.2006.09.086

Juslin, P. N., \& Vastfjall, D. (2008). Emotional responses to music: The need to consider underlying mechanisms. Behavioral and Brain Sciences, 31(5), 559-621. doi:10.1017/s0140525x08006079

Karremans, J. C., \& Verwijmeren, T. (2008). Mimicking attractive opposite-sex others: The role of romantic relationship status. Personality and Social Psychology Bulletin, 34(7), 939-950. doi:10.1177/0146167208316693

Kato, T., Takahashi, E., Sawada, K., Kobayashi, N., Watanabe, T., \& Ishii, T. (1983). A computer analysis of infant movements synchronized with adult speech. Pediatric Research, 17, 625-628.

Kendon, A. (1970). Movement coordination in social interaction: Some examples described. Acta Psychologica, 32(2), 101-125.

King, A. J., \& Cowlishaw, G. (2009). All together now: behavioural synchrony in baboons. Animal Behaviour, 78(6), 1381-1387.

Kirschner, S., \& Tomasello, M. (2009). Joint drumming: Social context facilitates synchronization in preschool children. Journal of Experimental Child Psychology, 102(3), 299-314. doi:10.1016/j.jecp.2008.07.005

Kirschner, S., \& Tomasello, M. (2010). Joint music making promotes prosocial behavior in 4-year-old children. Evolution and Human Behavior, 31, 354-364. 
Knoblich, G., Butterfill, S., \& Sebanz, N. (2011). Psychological research on joint action: Theory and data. In Brian Ross (Ed.), The psychology of learning and motivation (Vol. 54., Vol. 54, pp. 59-101). Burlington: Academic Press. doi:10.1016/B978-0-12-3855275.00003-6

Knoblich, G., \& Sebanz, N. (2008). Evolving intentions for social interaction: from entrainment to joint action. Philosophical Transactions of the Royal Society BBiological Sciences, 363(1499), 2021-2031. doi:10.1098/rstb.2008.0006

Kokal, I., Engel, A., Kirschner, S., \& Keysers, C. (2011). Synchronized drumming enhances activity in the caudate and facilitates prosocial commitment - if the rhythm comes easily. (A. Avenanti, Ed.)PLoS ONE, 6(11), e27272. doi:10.1371/journal.pone.0027272

Kollock, P. (1998). Social dilemmas: The anatomy of cooperation. Annual Review of Sociology, 24, 183-214.

Kramer, R. M., \& Brewer, M. B. (1984). Effects of group identity on resource use in a simulated commons dilemma. Journal of Personality and Social Psychology, 46(5), 1044-1057.

Kuper, H. (1947). An African aristocracy: Rank among the Swazi of Bechuanaland. London: Oxford University Press.

Kühnen, U., \& Hannover, B. (2000). Assimilation and contrast in social comparisons as a consequence of self- construal activation. European Journal of Social Psychology, 811, 799-811.

Kühnen, U., Hannover, B., \& Schubert, B. (2001). The semantic-procedural-interface model of the self: The role of self-knowledge for context-dependent versus contextindependent modes of thinking. Journal of Personality and Social Psychology, 80, 397409.

LaFrance, M. (1979). Nonverbal synchrony and rapport: Analysis by the cross-lag panel technique. Social Psychology, 42(1), 66-70.

LaFrance, M., \& Broadbent, M. (1976). Group rapport: Posture sharing as a nonverbal indicator. Group \& Organization Studies, 1(3), 328-333.

Lakens, D. (2010). Movement synchrony and perceived entitativity. Journal of Experimental Social Psychology, 45(5), 701-708.

Lakens, D., \& Stel, M. (2011). If they move in sync, they must feel in sync: Movement synchrony leads to attributions of rapport and entitativity. Social Cognition, 29, 1-14.

Lakin, J. L., \& Chartrand, T. L. (2003). Using nonconscious behavioral mimicry to create affiliation and rapport. Psychological Science, 14(4), 334-339.

Lakin, J. L., Chartrand, T. L., \& Arkin, R. M. (2008). I am too just like you - Nonconscious mimicry as an automatic behavioral response to social exclusion. Psychological Science, 19(8), 816-822. 
Lakin, J. L., Jefferis, V. E., Cheng, C. M., \& Chartrand, T. L. (2003). The chameleon effect as social glue: Evidence for the evolutionary significance of nonconscious mimicry. Journal of Nonverbal Behavior, 27(3), 145-162.

Lange, R. (1975). The nature of dance: An anthropological perspective. London: Macdonald and Evans.

Large, E. W., \& Palmer, C. (2002). Perceiving temporal regularity in music. Cognitive Science, 26, 1-37.

Leroi, A. M., \& Swire, J. (2006). The recovery of the past. World of Music, 48(3), 43-54.

Levitin, D. J. (2008). World in six songs: How the musical brain created human nature. New York: Dutton.

Lienhardt, G. (1957). Anuak Village Headmen. Africa: Journal of the International African Institute, 27(4), 341-355.

Lomax, A. (1968). Folk song style and culture. New Brunswick, N.J.: Transaction Books.

Lomax, A., \& Berkowit, N. (1972). Evolutionary taxonomy of culture. Science, 177(4045), 228-239.

London, J. (2004). Hearing in time: Psychological aspects of musical meter. Oxford: Oxford University Press.

Macrae, C. N., Duffy, O. K., Miles, L. K., \& Lawrence, J. (2008). A case of hand waving: Action synchrony and person perception. Cognition, 109(1), 152-156.

doi:10.1016/j.cognition.2008.07.007

Mann, N. I., Dingess, K. A., \& Slater, P. J. B. (2006). Antiphonal four-part synchronized chorusing in a neotropical wren. Biology Letters, 2, 1-4.

Markus, H. R., \& Kitayama, S. (1991). Culture and the self - implications for cognition, emotion, and motivation. Psychological Review, 98(2), 224-253.

Marsh, K. L., Richardson, M. J., \& Schmidt, R. C. (2009). Social connection through joint action and interpersonal coordination. Topics in Cognitive Science, 1, 320-339.

Marshall, D. A. (2002). Behavior, belonging, and belief: A theory of ritual practice. Sociological Theory, 20(3), 360-380.

Marshall, L. (1969). Medicine dance of Kung-bushmen. Africa, 39(4), 347-\&

Massie, T. M., Blasius, B., Weithoff, G., Gaedke, U., \& Fussmann, G. F. (2010). Cycles, phase synchronization, and entrainment in single-species phytoplankton populations. Proceedings of the National Academy of Sciences of the United States of America, 107(9), 4236-4241. doi:10.1073/pnas.0908725107 
Matthews, G., Jones, D. M., \& Chamberlain, A. G. (1990). Refining the measurement of mood: The UWIST Mood Adjective Checklist. British Journal of Psychology, 81(1), 1742 .

Maurer, R. E., \& Tindall, J. H. (1983). Effect of postural congruence on client's perception of counsellor empathy. Journal of Counselling Psychology, 30(2), 158-163.

Maynard Smith, J., \& Szathmary, E. (1995). The major transitions in evolution. Oxford: W.H. Freeman.

Mazzurega, M., Pavani, F., Paladino, M. P., \& Schubert, T. W. (2011). Self-other bodily merging in the context of synchronous but arbitrary-related multisensory inputs. Experimental Brain Research, 213-221. doi:10.1007/s00221-011-2744-6

McCroskey, L. L., McCroskey, J. C., \& Richmond, V. P. (2006). Analysis and improvement of the measurement of interpersonal attraction and homophily. Communication Quarterly, 54(1), 1-31.

McGrath, J. E., \& Kelly, J. R. (1986). Time and human interaction: Toward a social psychology of time. New York: The Guilford Press.

McNeill, W. H. (1995). Keeping together in time: Dance and drill in human history. Cambridge, MA: Harvard University Press.

Merker, B. (2000). Synchronous chorusing and human origins. In N. L. Wallin, B. Merker, \& S. Brown (Eds.), The origins of music (pp. 315-327). Cambridge, MA: MIT Press.

Merriam, A. P. (1964). The anthropology of music. Evanston: Northwestern University Press.

Miles, L. K., Griffiths, J. L., Richardson, M. J., \& Macrae, C. N. (2010). Too late to coordinate: Contextual influences on behavioral synchrony. European Journal of Social Psychology, 40(1), 52-60. doi:10.1002/ejsp.721

Miles, L. K., Lumsden, J., Richardson, M. J., \& Macrae, C. N. (2011). Do birds of a feather move together? Group membership and behavioral synchrony. Experimental Brain Research, 211(3-4), 495-503. doi:10.1007/s00221-011-2641-z

Miles, L. K., Nind, L. K., Henderson, Z., \& Macrae, C. N. (2010). Moving memories: Behavioral synchrony and memory for self and others. Journal of Experimental Social Psychology, 46(2), 457-460.

Miller, G. (2000). Evolution of human music through sexual selection. In N. Wallin, B. Merker, \& S. Brown (Eds.), The orgins of music (pp. 329-360). Cambridge MA: MIT Press.

Mithen, S. (2005). The singing Neanderthals: The origins of music, language, mind and body. London: Orion.

Moiseff, A., \& Copeland, J. (2010). Firefly synchrony: A behavioral strategy to minimize visual clutter. Science, 329(5988), 181. doi:10.1126/science.1190421 
Mullen, B., Brown, R., \& Smith, C. (1992). Ingroup bias as a function of salience, relevance, and status - An integration. European Journal of Social Psychology, 22(2), 103-122.

Muthén, B. O. (2004). Mplus technical appendices. Los Angeles, CA: Muthén \& Muthén.

Muthén, L. K., \& Muthén, B. O. (2010). Mplus user's guide. Version 6. Los Angeles, CA: Muthén \& Muthén.

Naruse, K., \& Hirai, T. (2000). Effects of slow tempo exercise on respiration, heart rate, and mood state. Perceptual and Motor Skills, 91(3), 729-740.

Nettl, B. (1983). The study of ethnomusicology: Twenty-nine issues and concepts. Urbana, IL: University of Illinois Press.

Newtson, D., Hairfield, J., Bloomingdale, I., \& Cutino, S. (1987). The Structure of action and interaction. Cognition, 5, 191-238.

Norenzayan, A., \& Heine, S. J. (2005). Psychological universals: What are they and how can we know? Psychological Bulletin, 131(5), 763-784.

North, A. C., Tarrant, M., \& Hargreaves, D. J. (2004). The effects of music on helping behavior: A field study. Environment and Behavior, 36(2), 266-275. doi:10.1177/0013916503256263

Nowak, M. A. (2006). Five rules for the evolution of cooperation. Science, 314(5805), 15601563. doi:10.1126/science. 1133755

Ones, U., \& Putterman, L. (2007). The ecology of collective action: A public goods and sanctions experiment with controlled group formation. Journal of Economic Behavior \& Organization, 62(4), 495-521. doi:10.1016/j.jebo.2005.04.018

Otten, S., \& Epstude, K. (2006). Overlapping mental representations of self, ingroup, and outgroup: unraveling self-stereotyping and self-anchoring. Personality \& Social Psychology Bulletin, 32(7), 957-69. doi:10.1177/0146167206287254

Oullier, O., \& Kelso, J. A. S. (2009). Coordination from the perspective of Social Coordination Dynamics. In R. A. Meyers (Ed.), Encyclopedia of complexity and system science (pp. 8198-8212). Berlin: Springer-Verlag.

Oullier, O., de Guzman, G. C., Jantzen, K. J., Lagarde, J., \& Kelso, J. A. S. (2008). Social coordination dynamics: Measuring human bonding. Social Neuroscience, 3(2), 178-192.

Over, H., \& Carpenter, M. (2009). Eighteen-month-old infants show increased helping following priming with affiliation. Psychological Science, 20(10), 1189-1193.

Oyserman, D., \& Lee, S. W. S. (2008). Does culture influence what and how we think? Effects of priming individualism and collectivism. Psychological Bulletin, 134 (2), 311342. 
Paladino, M.-P., Mazzurega, M., Pavani, F., \& Schubert, T. W. (2010). Synchronous multisensory stimulation blurs self-other boundaries. Psychological Science, 21(9), 1202-7. doi:10.1177/0956797610379234

Parncutt, R. (1994). A perceptual model of pulse salience and metrical accent in musical rhythms. Music Perception, 11, 409-464.

Patel, A. D. (2006). Musical rhythm, linguistic rhythm, and human evolution. Music Perception, 24(1), 99-104.

Patel, A. D., Iversen, J. R., Bregman, M. R., \& Schuiz, I. (2009). Experimental evidence for synchronization to a musical beat in a nonhuman animal. Current Biology, 19(10), 827830. doi:10.1016/j.cub.2009.03.038

Peretz, I. (2006). The nature of music from a biological perspective. Cognition, 100(1), 1-32. doi:10.1016/j.cognition.2005.11.004

Phillips-Silver, J., Aktipis, C. A., \& Bryant, G. A. (2010). The ecology of entrainment: Foundations of coordinated rhythmic movement. Music Perception, 28(1), 3-14. doi:10.1525/mp.2010.28.1.3

Phillips-Silver, J., \& Trainor, L. J. (2008). Vestibular influence on auditory metrical interpretation. Brain and Cognition, 67, 94-102.

Pinker, S. (1997). How the mind works. New York: Norton.

Powell, B. (2009). Japan, after the bubble. Time. Retrieved June 16, 2012, from http://www.time.com/time/specials/packages/article/0,28804,1902809_1902810_190519 2,00.html

Powers, M. (2005). The egalitarians - human and chimpanzee: An anthropological view of social organization. Cambridge, U.K: Cambridge University Press.

Preston, S. D., \& de Waal, F. B. M. (2002). Empathy: Its ultimate and proximate bases. Behavioral and Brain Sciences, 25(1), 1-71.

Prinz, W. (1997). Perception and action planning. European Journal of Cognitive Psychology, 9, 129-154.

Pronin, E. (2009). The introspection illusion. Advances in Experimental Social Psychology (Vol. 41, pp. 1-67). San Diego: Elsevier Academic Press Inc.

Radcliffe-Brown, A. R. (1948). The Andaman Islanders. Glencoe: The Free Press.

Rappaport, R. A. (1979). Ecology, meaning and religion. Richmond, CA: North Atlantic Books.

Repp, B. H. (2005). Sensorimotor synchronization: A review of the tapping literature. Psychonomic Bulletin and Review, 12, 969-992. 
Repp, B. H., \& Penel, A. (2004). Rhythmic movement is attracted more strongly to auditory than to visual rhythms. Psychological Research, 68(4), 252-270. doi:10.1007/s00426003-0143-8

Richardson, M. J., Marsh, K. L., Isenhower, R. W., Goodman, J. R. L., \& Schmidt, R. C. (2007). Rocking together: Dynamics of intentional and unintentional interpersonal coordination. Human Movement Science, 26(6), 867-891. doi:10.1016/j.humov.2007.07.002

Richardson, M. J., Marsh, K. L., \& Schmidt, R. C. (2005). Effects of visual and verbal interaction on unintentional interpersonal coordination. Journal of Experimental Psychology-Human Perception and Performance, 31(1), 62-79. doi:10.1037/00961523.31.1.62

Richerson, P. J., \& Boyd, R. (2005). Not by genes alone: How culture transformed human evolution. New York: Viking.

Roederer, J. (1984). The search for a survival value of music. Music Perception, 1, 350-356.

Rossano, M. J. (2009). Ritual behaviour and the origins of modern cognition. Cambridge Archaeological Journal, 19(2), 243-255. doi:10.1017/s0959774309000298

Rousseau, J. J. (1754). What is the origin of inequality among men, and is it authorised by natural law? (G. D. H. Cole, Trans.). Retrieved May 31, 2012, from http://www.constitution.org/jjr/ineq_04.htm

Ruffle, B. J., \& Sosis, R. (2007). Does it pay to pray? Costly ritual and cooperation. B. E. Journal of Economic Analysis \& Policy, 7(1), 37. doi:18

Rust, E. G. (1996). The music and dance of the world's religions : a comprehensive, annotated bibliography of materials in the English language. Westport, CT: Greenwood Press.

Rutter, D. R., \& Stephenson, G. M. (1977). The role of visual communication in synchronizing conversation. European Journal of Social Psychology, 7, 29-37.

Salimpoor, V. N., Benovoy, M., Larcher, K., Dagher, A., \& Zatorre, R. J. (2011). Anatomically distinct dopamine release during anticipation and experience of peak emotion to music. Nature Neuroscience, 14(2), 257-62. Nature Publishing Group. doi: $10.1038 / \mathrm{nn} .2726$

Schachner, A. (2010a). Auditory-motor entrainment in vocal mimicking species: Additional ontogenetic and phylogenetic factors. Communicative \& Integrative Biology, 3, 1-4.

Schachner, A. (2010b). The origins of human and avian auditory-motor entrainment. In A. Wessel, R. Menzel, \& G. Tembrock (Eds.), Quo Vadis, Behavioural Biology? Past, Present, and Future of an Evolving Science. Nova Acta Leopoldina. 
Schachner, A., Brady, T. F., Pepperberg, I. M., \& Hauser, M. D. (2009). Spontaneous motor entrainment to music in multiple vocal mimicking species. Current Biology, 19(10), 831-836.

Schachner, A., \& Carey, S. (2011). Goal inference for "irratonal" actions : The movements themselves are seen as the goal. Poster session presented at the annual meeting of the Cognitive Science Society, Boston, MA.

Scheflen, A. E. (1964). The significance of posture on communication systems. Psychiatry, 27, 316-331.

Schelling, T. (1960). The strategy of conflict. New York: Oxford University Press.

Schelling, T. (1973). Hockey helmets, concealed weapons, and daylight saving. A study of binary choices with externalities. Journal of Conflict Resolution, 17, 381-428.

Schmidt, R. C., Carello, C., \& Turvey, M. T. (1990). Phase-transitions and critical fluctuations in the visual coordination of rhythmic movements between people. Journal of Experimental Psychology-Human Perception and Performance, 16(2), 227-247.

Schmidt, R. C., \& O'Brien, B. (1997). Evaluating the dynamics of unintended interpersonal coordination. Ecological Psychology, 9(3), 189-206.

Schott, B. H., Minuzzi, L., Krebs, R. M., Elmenhorst, D., Lang, M., Winz, O. H., Seidenbecher, C. I., et al. (2008). Mesolimbic functional magnetic resonance imaging activations during reward anticipation correlate with reward-related ventral striatal dopamine release. The Journal of Neuroscience, 28(52), 14311-9. doi:10.1523/JNEUROSCI.2058-08.2008

Schubert, T. W., \& Otten, S. (2002). Overlap of self, ingroup, and outgroup: Pictorial measures of self-categorization. Self and Identity, 1(4), 353-376.

Sebanz, N., Bekkering, H., \& Knoblich, G. (2006). Joint action: bodies and minds moving together. Trends in Cognitive Sciences, 10(2), 70-76. doi:10.1016/j.tics.2005.12.009

Sebanz, N., \& Knoblich, G. (2009). Prediction in Joint Action: What, When, and Where. Topics in Cognitive Science, 1(2), 353-367. doi:10.1111/j.1756-8765.2009.01024.x

Seger, C. R., Smith, E. R., \& Mackie, D. M. (2009). Subtle activation of a social categorization triggers group-level emotions. Journal of Experimental Social Psychology, 45(3), 460-467. doi:10.1016/j.jesp.2008.12.004

Semin, G. R., \& Cacioppo, J. T. (2008). Grounding social cognition: Synchronization, coordination, and co-regulation. In G. R. Semin \& E. R. Smith (Eds.), Embodied grounding: Social, cognitive, affective, and neuroscientific approaches. New York: Cambridge University Press.

Sforza, A., Bufalari, I., Haggard, P., \& Aglioti, S. M. (2010). My face in yours: visuo-tactile facial stimulation influences sense of identity. Social Neuroscience, 5, 148-162. 
Shariff, A. F., \& Norenzayan, A. (2007). God is watching you - Priming god concepts increases prosocial behavior in an anonymous economic game. Psychological Science, 18(9), 803-809.

Shaw, D. (2012). It's like looking in a mirror. Poster session presented at the past, present, and future in the scientific study of religion conference, Brno, Czech Republic.

Shockley, K., Baker, A. A., Richardson, M. J., \& Fowler, C. A. (2007). Articulatory constraints on interpersonal postural coordination. Journal of Experimental PsychologyHuman Perception and Performance, 33(1), 201-208. doi:10.1037/0096-1523.33.1.201

Shockley, K., Santana, M. V., \& Fowler, C. A. (2003). Mutual interpersonal postural constraints are involved in cooperative conversation. Journal of Experimental Psychology-Human Perception and Performance, 29(2), 326-332.

Skyrms, B. (2004). The stag hunt and the evolution of social structure. Cambridge, UK: Cambridge University Press.

Smith, E. R. (2008). An embodied account of self-other "overlap" and its effects. In G. R. Semin \& E. R. Smith (Eds.), Embodied grounding: Social, cognitive, affective, and neuroscientific approaches. New York: Cambridge University Press.

Smith, E. R., \& Henry, S. (1996). An in-group becomes part of the self: Response time evidence. Personality and Social Psychology Bulletin, 22(6), 635-642. doi:10.1177/0146167296226008

Snoek, J. (2006). Defining "rituals.” In J. Kreinath, J. Snoek, \& M. Stausberg (Eds.), Theorizing rituals: Issues, topics, approaches, concepts. Leiden: Brill.

Spencer, M. A., Swallow, S. K., Shogren, J. F., \& List, J. A. (2009). Rebate rules in threshold public good provision. Journal of Public Economics, 93, 798-806. Elsevier B.V.

Spencer, P. (1985). Interpretations of the dance in anthropology. In P. Spencer (Ed.), Society and the dance: the social anthropology of process and performance (pp. 1-46). Cambridge, U.K: Cambridge University Press.

Stapel, D. A., \& Koomen, W. (2005). Competition, cooperation, and the effects of others on me. Journal of Personality and Social Psychology, 88(6), 1029-1038. doi:10.1037/00223514.88.6.1029

Stel, M., Van Baaren, R. B., \& Vonk, R. (2008). Effects of mimicking: Acting prosocially by being emotionally moved. European Journal of Social Psychology, 38(6), 965-976. doi:10.1002/ejsp.472

Stel, M., \& Vonk, R. (2010). Mimicry in social interaction: Benefits for mimickers, mimickees, and their interaction. British Journal of Psychology, 101, 311-323. doi:10.1348/000712609x465424 
Stevens, J. R., Cushman, F. a., \& Hauser, M. D. (2005). Evolving the psychological mechanisms for cooperation. Annual Review of Ecology, Evolution, and Systematics, 36(1), 499-518. doi:10.1146/annurev.ecolsys.36.113004.083814

Street, R. L. (1984). Speech convergence and speech evaluation in fact finding interviews. Human Communication, 11, 139-169.

Strogatz, S. (2003). Sync: The emerging science of spontaneous order. New York: Hyperion.

Swann, W. B., Gomez, A., Seyle, D. C., Morales, J. F., \& Huici, C. (2009). Identity fusion: The interplay of personal and social identities in extreme group behavior. Journal of Personality and Social Psychology, 96(5), 995-1011. doi:10.1037/a0013668

Thoren, P., Floras, J. S., Hoffmann, P., \& Seals, D. R. (1990). Endorphins and exercise: Physiological mechanisms and clinical implications. Medicine and Science in Sports and Exercise, 22, 417-428.

Tickle-Degnen, L., \& Rosenthal, R. (1990). The nature of rapport and its nonverbal correlates. Psychological Inquiry, 1(4), 285-293.

Todd, N. P. M., Lee, C. S., \& O’Boyle, D. J. (2002). A sensorimotor theory of temporal tracking and beat induction. Psychological Research, 66, 26-39.

Tomasello, M., \& Carpenter, M. (2007). Shared intentionality. Developmental science, 10(1), 121-5. doi:10.1111/j.1467-7687.2007.00573.x

Tomasello, M., Carpenter, M., Call, J., Behne, T., \& Moll, H. (2005). Understanding and sharing intentions: The origins of cultural cognition. Behavioral and Brain Sciences, 28(5), 675-735.

Trainor, L. J., Gao, X., Lei, J., Lehtovaara, K., \& Harris, L. R. (2009). The primal role of the vestibular system in determining musical rhythm. Cortex, (45), 35-43.

Trivers, R. L. (1971). The evolution of reciprocal altruism. Quarterly Review of Biology, 46, $35-57$.

Turner, J. C. (1984). Social identification and psychological group formation. In H. Tajfel (Ed.), The social dimension: European developments in social psychology (pp. 518538). Cambridge, U.K: Cambridge University Press.

Turner, J. C., Hogg, M. A., Oakes, P. J., Reicher, S. D., \& Wetherell, M. S. (1987). Rediscovering the social group: A self-categorization theory. Oxford, UK: Blackwel.

Turner, V. (1964). The forest of symbols: Aspects of Ndembu ritual. Ithaca: Cornell University Press.

Turner, V. (1969). The ritual process: Structure and anti-structure. Chicago, IL: Aldine. 
Utz, S. (2004). Self-construal and cooperation: Is the interdependent self more cooperative than the independent self? Self and Identity, 3(3), 177-190. doi:10.1080/13576500444000001

Valdesolo, P., \& DeSteno, D. (2011). Synchrony and the social tuning of compassion. Emotion, 11(2), 262-266.

Valdesolo, P., Ouyang, J., \& DeSteno, D. (2010). The rhythm of joint action: Synchrony promotes cooperative ability. Journal of Experimental Social Psychology, 46(4), 693695.

Van Baaren, R. B., Holland, R. W., Kawakami, K., \& van Knippenberg, A. (2004). Mimicry and prosocial behavior. Psychological Science, 15(1), 71-74.

Van Baaren, R. B., Holland, R. W., Steenaert, B., \& van Knippenberg, A. (2003). Mimicry for money: Behavioral consequences of imitation. Journal of Experimental Social Psychology, 39(4), 393-398. doi:10.1016/s0022-1031(03)00014-3

Van Baaren, R. B., Horgan, T. G., Chartrand, T. L., \& Dijkmans, M. (2004). The forest, the trees, and the chameleon: Context dependence and mimicry. Journal of Personality and Social Psychology, 86(3), 453-459. doi:10.1037/0022-3514.86.3.453

Van Lange, P. A. M. (2008). Does empathy trigger only altruistic motivation? How about selflessness or justice? Emotion, 8(6), 766-774. doi:10.1037/a0013967

Van Ulzen, N. R., Lamoth, C. J. C., Daffertshofer, A., Semin, G. R., \& Beek, P. J. (2008). Characteristics of instructed and uninstructed interpersonal coordination while walking side-by-side. Neuroscience Letters, 432(2), 88-93. doi:10.1016/j.neulet.2007.11.070

Vesper, C., Butterfill, S., Knoblich, G., \& Sebanz, N. (2010). A minimal architecture for joint action. Neural Networks, 23, 998-1003. doi:10.1016/j.neunet.2010.06.002

Vesper, C., van der Wel, R. P. R. D., Knoblich, G., \& Sebanz, N. (2011). Making oneself predictable: Reduced temporal variability facilitates joint action coordination. Experimental Brain Research., 211, 517-530. doi:10.1007/s00221-011-2706-z

Wallin, N., Merker, B., \& Brown, S. (Eds.). (2000). The origins of music. Cambridge, MA: MIT press.

Warner, R. M., Malloy, D., Schneider, K., Knoth, R., \& Wilder, B. (1987). Rhythmic organization of social interaction and observer ratings of positive affect and involvement. Journal of Nonverbal Behavior, 11(2), 57-74.

West, S. A., El Mouden, C., \& Gardner, A. (2011). Sixteen common misconceptions about the evolution of cooperation in humans. Evolution and Human Behavior, 32, 231-262.

West, S. A., Griffin, A. S., \& Gardner, A. (2007). Social semantics: altruism, cooperation, mutualism, strong reciprocity and group selection. Journal of Evolutionary Biology, 20(2), 415-432. doi:10.1111/j.1420-9101.2006.01258.x 
Wickler, W. (1976). Biological significance of stationary and nonstationary processes. Journal of Theoretical Biology, 61, 493-497.

Wiekens, C. J., \& Stapel, D. A. (2008). The effects of self-construal level on diversity. Social Cognition, 26, 368-377. doi:10.1521/soco.2008.26.3.368

Wilson, D. S., \& Sober, E. (1994). Re-introducing group selection to the human behavioral sciences. Behavioral and Brain Sciences, 17, 585-654.

Wilson, D. S., Tumminelli, D., Brien, O., \& Sesma, A. (2009). Human prosociality from an evolutionary perspective: Variation and correlations at a city-wide scale. Evolution and Human Behavior, 30(3), 190-200. Elsevier Inc. doi:10.1016/j.evolhumbehav.2008.12.002

Wilson, M., \& Wilson, T. P. (2005). An oscillator model of the timing of turn-taking. Psychonomic Bulletin and Review, 12, 957-968.

Wiltermuth, S. S. (2012). Synchronous activity boosts compliance with requests to aggress. Journal of Experimental Social Psychology, 48(1), 453-456. doi:10.1016/j.jesp.2011.10.007

Wiltermuth, S. S., \& Heath, C. (2009). Synchrony and cooperation. Psychological Science, 20(1), 1-5. doi:10.1111/j.1467-9280.2008.02253.x

Yamagishi, T., \& Mifune, N. (2009). Social exchange and solidarity: In-group love or outgroup hate? Evolution and Human Behavior, 30(4), 229-237. doi:10.1016/j.evolhumbehav.2009.02.004

Ybarra, O., \& Trafimow, D. (1998). How priming the private self or collective self affects the relative weights of attitudes and subjective norms. Personality and Social Psychology Bulletin, 24, 362-370.

Zak, P. J., Kurzban, R., \& Matzner, W. T. (2005). Oxytocin is associated with human trustworthiness. Hormones and Behavior, 48(5), 522-527. doi:10.1016/j.yhbeh.2005.07.009

Zak, P. J., Stanton, A. A., \& Ahmadi, S. (2007). Oxytocin increases generosity in humans. PLoS One, 2(11), e1128. Retrieved from //medline: 17987115

Zatorre, R. J., Chen, J. L., \& Penhune, V. B. (2007). When the brain plays music: Auditorymotor interactions in music perception and production. Nature Reviews Neuroscience, 8 , $547-558$.

Zentner, M., \& Eerola, T. (2010). Rhythmic engagement with music in infancy. Proceedings of the National Academy of Sciences of the United States of America, 107, 5768-5773.

Zivotofsky, A. Z., \& Hausdorff, J. M. (2007). The sensory feedback mechanisms enabling couples to walk synchronously: An initial investigation. Journal of Neuroengineering and Rehabilitation, 4, 5. doi:28 10.1186/1743-0003-4-28 


\section{Appendices}

\section{Appendix 1: List of words used in experiment 2}

$\begin{array}{llll}\text { rock } & \text { stool } & \text { seat } & \text { quart } \\ \text { cork } & \text { trunk } & \text { bowl } & \text { square } \\ \text { lamp } & \text { stove } & \text { sphere } & \text { odd } \\ \text { jug } & \text { key } & \text { bus } & \text { tank } \\ \text { foot } & \text { farm } & \text { vest } & \text { lawn } \\ \text { pig } & \text { plain } & \text { frog } & \text { shy } \\ \text { dark } & \text { name } & \text { nun } & \text { cow } \\ \text { hat } & \text { phase } & \text { part } & \text { fur } \\ \text { chin } & \text { horse } & \text { rain } & \text { street } \\ \text { coarse } & \text { tool } & \text { cord } & \text { ship } \\ \text { knot } & \text { clock } & \text { spray } & \text { slush } \\ \text { glass } & \text { fork } & \text { door } & \text { hay } \\ \text { chair } & \text { stiff } & \text { book } & \text { bench } \\ \text { bland } & \text { egg } & \text { ink } & \text { month } \\ \text { black } & \text { truck } & \text { arm } & \end{array}$




\section{Appendix 2: Instructions for the public goods game}

Group investment game

You have already received $\$ 5$ for showing up on time to this experiment. This will be given to you at the end of the study. We will now play a game for which you will be given a further $\$ 5$ with the opportunity to earn more. The money you earn will be paid to you, in cash, at the end of the experiment after a questionnaire. The decision you and the other participants make will affect how much money you earn, so please read these instructions carefully. Please do not talk to the other participants. If you have any questions please ask me directly.

Instructions

1. This game involves four players. The other players are the people you have just done a group activity with.

2. Each player in your group has been given $\$ 5.00$ to play this game.

3. Each person has the opportunity to contribute, confidentially, some or all of all of this $\$ 5$ to a 'group investment'. All money in the group investment will be doubled and then divided equally among all members of your group.

4. Alternatively, you may keep as much of your money as you like for yourself.

5. Your task is to decide how much money you would like to place in the group investment. You may choose any 50c increment between 0 and $\$ 5$ (i.e. $\$ 0.50, \$ 1.00$, $\$ 1.50$ etc). There is no right or wrong answer. Choose whatever you would prefer

6. Your earnings will be the sum of the amount of money you decide to keep yourself plus your equal share of the group investment.

Your decision will be confidential. The other participants will never know how much you contributed.

There is no deception in this game. You will receive the correct amount of money promised.

Here are some examples of how the game could play out:

- If everyone keeps all of their money then everyone will receive $\$ 5.00$ each from this investment game.

- If everyone puts all of their money into the group investment, everyone will receive $\$ 10$ each $(4 \times \$ 5=\$ 20$, doubled $=\$ 40$, divided by 4$)$.

- If the other players contribute all of their money to the group investment and only you keep all of your money you will earn $\$ 12.50$, and the other players $\$ 7.50$.

- If you contribute all of your money to the group investment but everyone else keeps all of their's, then you will earn $\$ 2.50$, and the other players $\$ 7.50$. 
A quick question to check you understand the rules:

For example: you decide to keep $\$ 2.50$ and contribute $\$ 2.50$ to the group investment. The other three players also decide to put $\$ 2.50$ each into the group investment. How much will you earn?

Remember: Your total earnings $=$ Earnings you keep + earning from the group investment

Earnings from the money $Y O U$ KEEP $=\$$

Earnings from GROUP INVESTMENT:

- All 4 players put $\$ 2.50$ into the group account, this will result in a total of $\$$

- We then double this to get $\$$

- We then divide this number equally between the 4 players to get $\$$ each (B)

- Your total earnings $=$ Earnings you keep $(\mathrm{A})+$ earning from the group investment $(\mathrm{B})=$ $\$$ 


\title{
Appendix 3: Foot-pedal timing analysis
}

\author{
Method
}

Why use circular statistics?

Following previous studies that looked to measure entrainment (Kirschner \& Tomasello, 2009; Oullier, de Guzman, Jantzen, Lagarde, \& Kelso, 2008; Schachner et al., 2009) I used circular statistics (Fisher, 1993) to assess synchrony. Circular statistics is based on data where both ends of a scale meet, unlike in linear scales where either end of the scale are polar opposites. Although it is possible to use a linear scale of asynchrony deviation, measured in seconds, such data doesn't take into account the cyclical nature of the data: a particular foot-pedal press by participant A could be later than participant B's previous footpedal press, or earlier than participant B's next foot-pedal press. For example, say participant B pressed their foot-pedal at 2 seconds after the start of the experiment and their next footpedal press was at 4 seconds. If participant A presses their foot-pedal at 3.5 seconds, this foot-pedal press could have a asynchrony deviation of 1.5 seconds when compared to participant B's first foot-pedal press, or an asynchrony deviation of 0.5 seconds compared to participant B's second foot-pedal press. Using circular statistics, participant A can instead be said to be $240^{\circ}$ or $1.5 \pi$ out of phase with participant B. Using this measure of phase deviation also allows for asynchronies to be standardised to the same scale. This is important in this study where participants are moving at different speeds.

\section{Calculation of phase deviation}

Previous synchrony studies using circular statistics compared how well one rhythmic source matched another rhythmic source with a fixed inter-stimulus interval. In this study, I had 3 rhythmic sources with the inter-stimulus interval fluctuating between foot-pedal presses. To overcome these problems, for each participant's foot-pedal press I calculated the relative phase deviation $\theta$ from each of the other participants foot presses at that particular time point. For each participant, each foot-pedal press at time point $t$ was matched up to the appropriate interval between foot-pedal presses (inter-stimulus interval [ISI]) for each of the other participants. This was done in pairs, such that each time point for participant A was matched onto the appropriate inter-stimulus interval for B and then the appropriate ISI for C; 
each time point for $\mathrm{B}$ was matched onto the appropriate ISI of A and C; and similar for participant $C$. This resulted in six columns of $\theta$ for each group: A matched on to $B(A B), A$ matched on to $\mathrm{C}$ (AC), B matched on to A (BA), B matched on to $\mathrm{C}(\mathrm{BC}), \mathrm{C}$ matched on to A (CA), and $\mathrm{C}$ matched on to $\mathrm{B}(\mathrm{CB})$. Note that due to the varying length of ISI, especially during the asynchrony conditions, A matched onto B (AB) was not the same as B matched on A (BA). The ISI interval was calculated by finding the foot-pedal presses of the matched participant that were just before and just after time point $t$. An asynchrony deviation was calculated by subtracting the earlier time point of the ISI from $t$. This asynchrony deviation was then converted into $\theta$ (in degrees) by multiplying it by $360^{\circ}$ and then dividing by the ISI. For example, to find the relative phase match of participant A compared to participant B at a foot-pedal press at time $t$ by participant $\mathrm{A}\left(\mathrm{t}_{\mathrm{A}}\right)$, the following equation was used, where $\mathrm{t}_{\mathrm{B} 1}$ is the maximum time point of a foot-pedal press by participant $B$ that is less than $t_{A}$, and $t_{B 2}$ is the minimum time point of a foot-pedal press by participant $B$ that is greater than $t_{A}$ :

$$
\theta=\frac{\left(t_{A}-t_{B 1}\right) \times 360^{\circ}}{\left(t_{B 2}-t_{B 1}\right)}
$$

Calculation of the mean phase deviation

With circular data, the arithmetic mean for $\theta$ isn't appropriate. For example the mean of $30^{\circ}$ and $330^{\circ}$ is not $180^{\circ}$, but $0^{\circ}$. Instead, mean phase deviation (or mean direction, $\bar{\theta}$ ) is used, which is calculated by dividing the sum of the sine of each $\theta$ (converted to radians) by the sum of the cosine of each $\theta$ and then calculating the arctan (Fisher, 1993):

$$
\begin{gathered}
S=\sum_{i=1}^{n} \sin \theta_{i}, C=\sum_{i=1}^{n} \cos \theta_{i} \\
\bar{\theta}\left\{\begin{array}{lr}
\tan ^{-1}\left(\frac{S}{C}\right) & S>0, C>0 \\
\tan ^{-1}\left(\frac{S}{C}\right)+\pi & C<0 \\
\tan ^{-1}\left(\frac{S}{C}\right)+2 \pi & S<0, C>0
\end{array}\right.
\end{gathered}
$$


As well as $\theta$, each data point being a vector also has a length $\mathrm{R}$. This is calculated by (Fisher, 1993): $\mathrm{R}^{2}=\mathrm{C}^{2}+\mathrm{S}^{2}$. The mean resultant length $\bar{R}$ is calculated by (Fisher, 1993): $\bar{R}=R / n$, where $n$ is the number of foot-pedal presses. $\bar{R}$ varies from 0 to 1 and is the inverse of the circular variance $(V)$, (Fisher, 1993): $\mathrm{V}=1-\bar{R}$, such that an $\bar{R}$ of 1 means that all data points are coincident. However, an $\bar{R}$ of 0 doesn't not necessarily equate to a uniform distribution. A perfectly bimodal distribution with data points being coincident on a particular value on opposite sides of the circle (for example $0^{\circ}$ and $180^{\circ}$ ) would also produce an $\bar{R}$ of 0 .

\section{Statistical tests for the distribution of the phase deviation}

Although $\bar{R}$ gives some indication of dispersion, it is important to assess the distribution for how concentrated data points are. To statistically assess distribution the Rayleigh test can be used. This procedure tests the null hypothesis of uniform distribution of data points against an alternative hypothesis of a unimodal distribution with an unspecified mean. The $V$-test can be used to test for a unimodal distribution with a specified mean against the null hypothesis of uniformity.

For each participant, $\bar{\theta}$ and $\bar{R}$ was calculated across both of the other participants in the group, i.e., $\bar{\theta}$ and $\bar{R}$ for participant $\mathrm{A}$ was a combination of the $\theta$ for the comparison $\mathrm{AB}$ and the $\theta$ for the comparison AC. This allowed for an overall measure of synchrony as experienced by that participant.

\section{Hypotheses}

For the synchrony condition, it was hypothesised that:

1a. $\bar{R}$ for all participants in the SyGG and SyIG conditions will be close to 1 . An $\bar{R}$ close to 1 will indicate there is very little variance in the phase distribution between participants in a group, indicating that participants are entrained.

1b. Participants in both the SyGG and SyIG conditions should have a unimodal distribution with $\bar{\theta}$ centred on $0^{\circ}$. This will indicate that participants are in-phase with each other.

For the asynchrony condition, it was hypothesised that: 
2a. $\overline{\mathrm{R}}$ for all participants in the AsGG and AsIG conditions will be close to 0 .

2b. Participants in both the AsGG and AsIG conditions should have a uniform distribution of $\bar{\theta}$ (The Rayleigh test will not be significant) and the distribution of $\bar{\theta}$ should not be centred on $0^{\circ}$ (the $V$-test is not significant).

For the sequential condition, a 6/8 rhythm was used with participant A moving on the $1^{\text {st }}$ beat, participant $\mathrm{B}$ on the $3^{\text {rd }}$ beat, and participants $\mathrm{C}$ on the $5^{\text {th }}$ beat. This meant that participant B should have moved a third of the rhythmic phase after A, and participant C two thirds of the rhythm phase. Likewise, in comparison to B, C moves a third of the rhythmic phase after B, and A two thirds of the rhythm phase after B. The sequential condition should therefore result in one of the participants being $120^{\circ}$ out of phase and the other being $240^{\circ}$ out of phase with the participant of interest. As both the matched participants are included in the calculation of a participant's $\bar{\theta}$, this would result in a bimodal distribution, a lower $\bar{R}$, and therefore suggesting more dispersal than actually present. To create a unimodal distribution the $\theta$ for the participant that would be $120^{\circ}$ out of phase was subtracted from $360^{\circ}$. Therefore, it was hypothesised in the sequential condition that:

3a. $\bar{R}$ for all participants in both the SqGG and SqIG conditions will be close to 1 .

3b. Participants in both the SqGG and SqIG conditions should have a unimodal distribution with $\bar{\theta}$ centred on $240^{\circ}$.

As well as examining synchrony within a condition, it is also important to examine synchrony between conditions. Because $\bar{R}$ is on a linear scale from 0 to 1 , linear parametric statistics were used to test for differences in measured synchrony in the overall mean $\bar{R}$ between conditions (Kirschner \& Tomasello, 2009). Because I have precise a priori comparisons (outlined below), t-tests rather than omnibus ANOVAs were used to examine differences.

It was hypothesised that:

4a. The overall mean $\overline{\mathrm{R}}$ (i.e., the variance in phase matching) for SyGG and the overall mean $\overline{\mathrm{R}}$ for SyIG should not be significantly different.

4b. The overall mean $\bar{R}$ for AsGG and the overall mean $\bar{R}$ for AsIG should not be significantly different.

4c. The overall mean $\overline{\mathrm{R}}$ for GSq and the overall mean $\overline{\mathrm{R}}$ for ISq should not be significantly different. 
5a. The overall mean $\overline{\mathrm{R}}$ for the synchrony condition should be significantly different from asynchrony condition (where there should be no phase relationship),

5 b. The overall mean $\overline{\mathrm{R}}$ for synchrony condition should not be significantly different from sequential condition (where there should be a unimodal phase relationship centred at $\left.240^{\circ}\right)$

5c. The overall mean $\overline{\mathrm{R}}$ for sequential condition should be significantly different from asynchrony condition.

\section{Results}

The programme recording the time stamps from the foot-pedals malfunctioned on one of the SqIG trials so data from only 80 participants was used in the analyses of foot-pedal data.

\section{Synchrony condition}

A vector diagram (Figure 26 on page 147) for the synchrony condition shows that the mean direction $\bar{\theta}$ (mean phase difference) and mean distance $\bar{R}$ (variance in phase difference) for each participant shows a similar distribution of $\bar{\theta}$ for both the group goal and individual goal conditions. $\bar{R}$ for all participants was above 0.9 suggesting minimal variance in phase angle. The Rayleigh and $V$-tests for all participants in the synchrony condition were highly significant $(p<.001)$ indicating that the data was unimodal and centred on $0^{\circ}$. The overall mean $\bar{R}$ for SyGG $(M=0.94, S D=0.02)$ was significantly lower than the overall mean $\bar{R}$ for SyGG $(M=0.96, S D=0.03), t(26)=2.95, p=.01, d=1.15$. This suggests that the synchronisation in the group goal condition was not as good as in the individual goal condition. The overall mean $\bar{R}$ for synchrony condition $(M=0.95, S D=0.03)$ was significantly higher than the asynchrony condition $(M=0.07, S D=0.07), t(32.16)=58.28, p$ $<.01, d=16.34$; but not significantly different from the sequential condition $(M=0.96, S D=$ $0.04), t(51)=1.40, p=.17, d=.39$. 


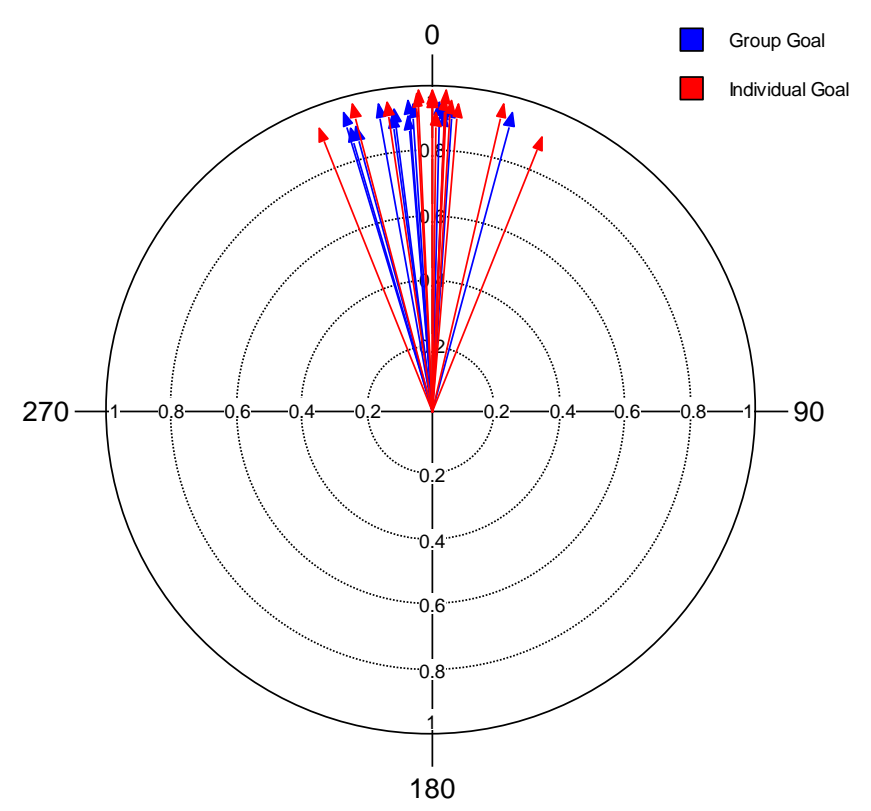

Figure 26. Vector diagram showing the distribution of $\bar{\theta}$ for each participant in the synchrony condition. Each arrow corresponds to a participant with the angle of the arrow representing $\bar{\theta}$, and the length of the arrow representing $\bar{R}$. Blue arrows are participants who were in the group goal condition (SyGG); red arrows are participants who were in the individual goal condition (SyIG). The numbers outside of the circle represent $\bar{\theta}$ in degrees. The numbers inside of the circle represent the vector length $\bar{R}$.

\section{Asynchrony condition}

Comparing Figure 26 to Figure 27 (page 148), we can see that for all asynchrony groups that $\bar{R}$ was considerable smaller than for synchrony groups suggesting that participants in the asynchrony groups had minimal entrainment to each other. The Rayleigh and $V$-tests for all participants in the AsIG condition was not significant $(p>.05)$ indicating that the data was uniformly distributed. $\bar{R}$ for all participants was below 0.05 . However, the Rayleigh test for 5 participants in the AsGG condition was significant $(p<.001)$, suggesting the data was not uniform. $V$-tests examining if the data was centred on a mean of $0^{\circ}$ were not significant. $V$-tests examining if the data was centred on a mean of $180^{\circ}$ were significant for all five participants. This suggests that to help stay out of time, some participants moved antiphase with another participant. Visual inspection of the distribution of $\theta$ for these participants showed that $\theta$ was still very dispersed. This indicates that although there was a slight trend towards anti-phase coordination, the movement was still highly asynchronous (see Figure 28 
on page 149). The overall mean $\bar{R}$ for AsGG $(M=0.12, S D=0.08)$ was significantly higher than the overall mean $\bar{R}$ for $\operatorname{AsIG}(M=0.03, S D=0.01), t(13.95)=4.31, p<.01, d=1.58$. This suggests that degree of synchrony in the AsGG condition was greater than in the AsIG condition (i.e., AsIG was more asynchronous). The overall mean $\bar{R}$ for the asynchrony condition was significantly different from sequential condition, $t(42.37)=53.57, p<.01, d=$ 15.61 .

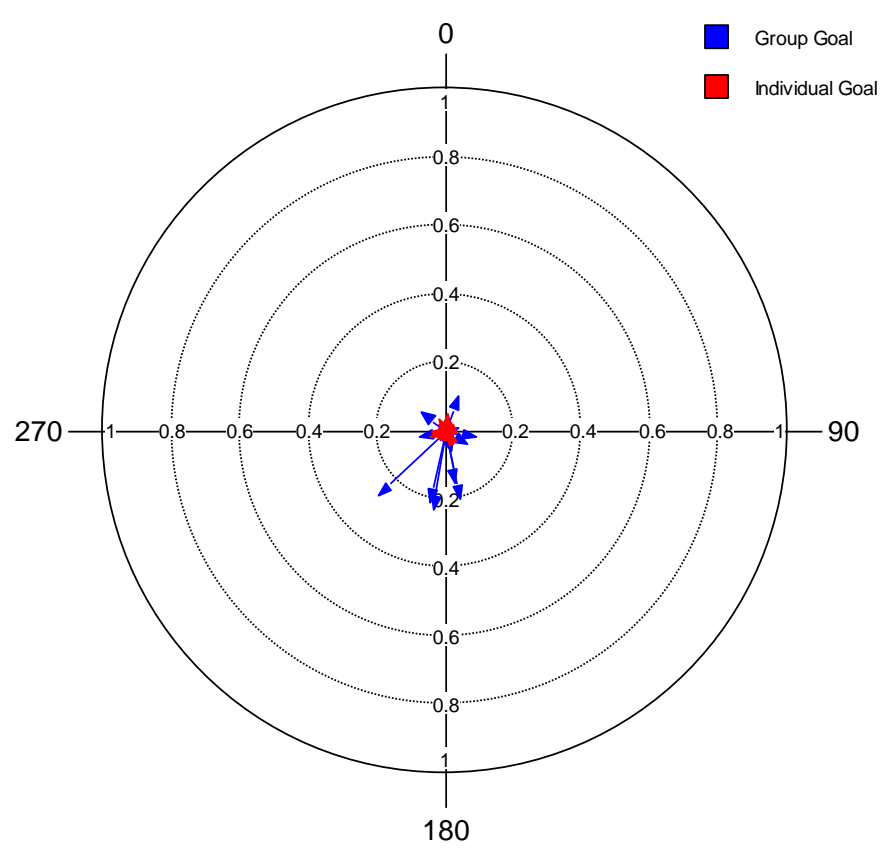

Figure 27. Vector diagram showing the distribution of $\bar{\theta}$ for each participant in the asynchrony condition. 


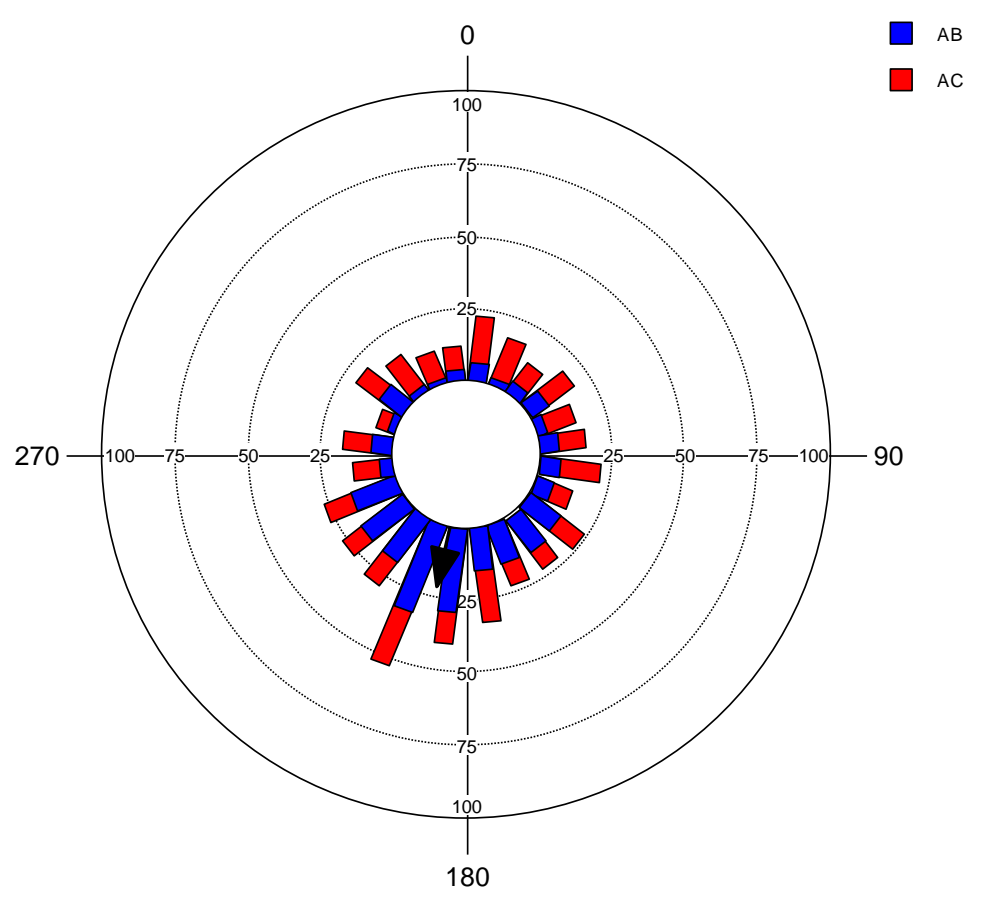

Figure 28. Angular histogram showing the distribution of the relative phase angle $\theta$ for each pedal press for a participant in the asynchrony group goal condition (AsGG). The numbers outside of the circle represent $\theta$ in degrees. The numbers inside of the circle represent frequency. This participant was chosen as an example because they had the highest $\bar{R}$ in the AsGG condition. The colour shading represents the comparison with a particular group member (blue represents the relative phase of participant A to participant B; red, represents participant A to participant $\mathrm{C}$ ). The black arrow represents the mean phase $\bar{\theta}$ for this participant.

\section{Sequential condition}

Figure 29 (page 150) shows that both the group goal and individual goal conditions have a similar distribution of $\bar{\theta} . \bar{R}$ for all participants was above 0.8 . The Rayleigh and $V$ tests for all participants in the SqGG and SqGG conditions were highly significant $(p<.001)$ indicating that the data was unimodal and centred on $240^{\circ}$. The overall mean $\bar{R}$ for SqGG (M $=0.97, S D=0.02)$ was not significantly different to the overall mean $\bar{R}$ for ISq $(M=0.95, S D$ $=0.06), t(11.14)=1.16, p=0.27, d=0.45$. 


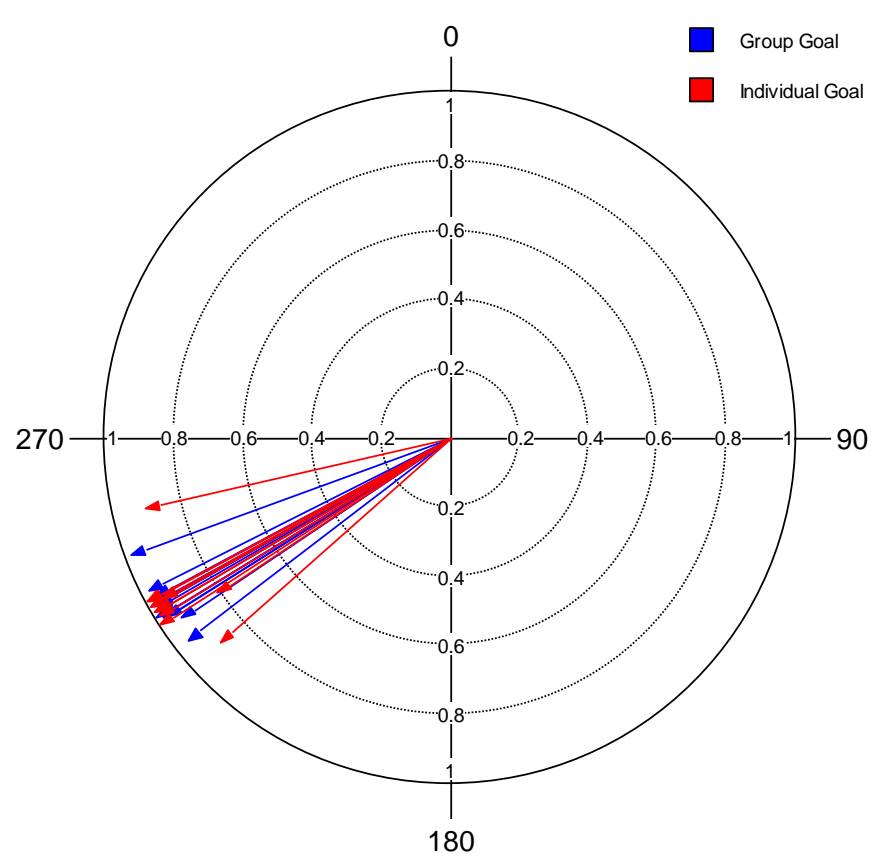

Figure 29. Vector diagram showing the distribution of $\bar{\theta}$ for each participant in the sequential condition. 